Aus der chirurgischen Universitätsklinik

Abteilung für Allgemeine- und Viszeralchirurgie mit Poliklinik

Albert-Ludwigs-Universität Freiburg i. Br.

Ärztl. Direktor: Prof. Dr. Dr. h.c. U.T. Hopt

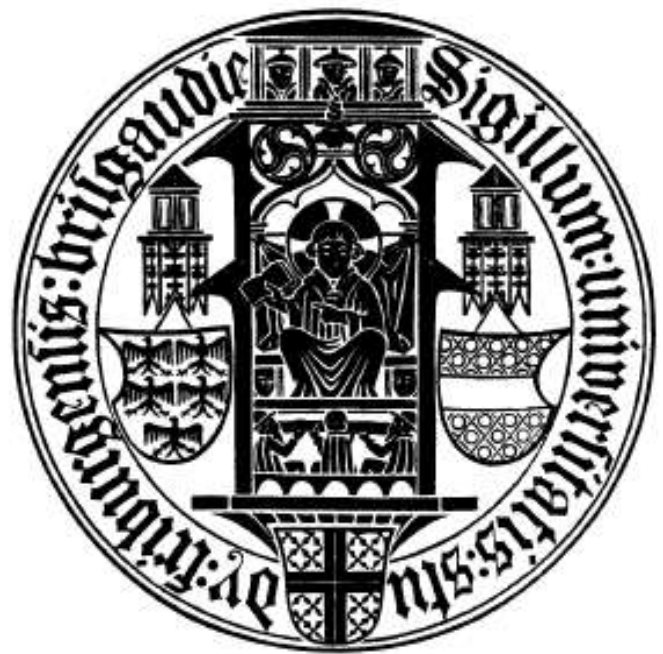

\title{
Neoadjuvante Chemotherapie (PELF) beim Magenkarzinom
}

\author{
INAUGURAL-DISSERTATION \\ zur \\ Erlangung des Medizinischen Doktorgrades \\ der Medizinischen Fakultät \\ der Albert-Ludwigs-Universität \\ Freiburg i. Br. \\ Vorgelegt 2010 \\ von \\ Catharina Ruf \\ geboren in Freiburg im Breisgau
}


Meinen Eltern und Geschwistern gewidmet

Dekan: $\quad$ Prof. Dr. med. C. Peters

1. Gutachter:

Prof. Dr.med. O. Thomusch

2. Gutachter: PD Dr. G. Illerhaus

Jahr der Promotion: $\quad 2010$ 


\section{Inhaltsverzeichnis}

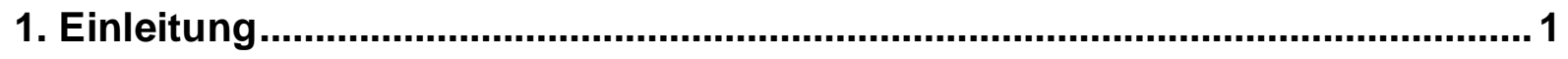

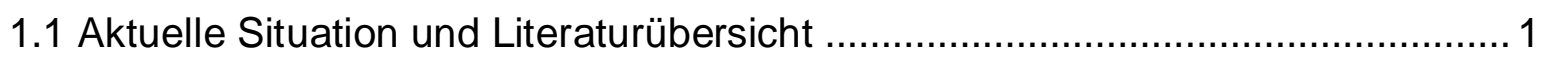

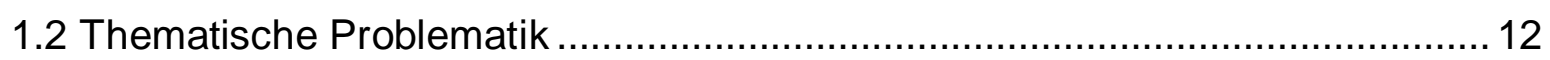

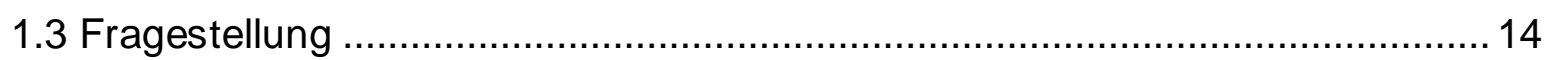

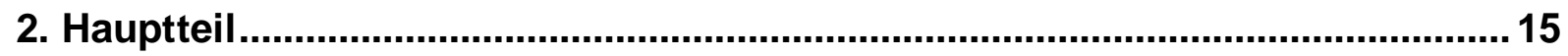

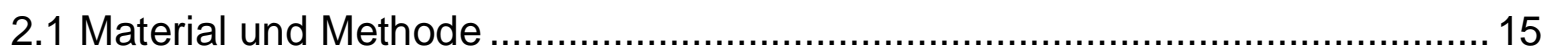

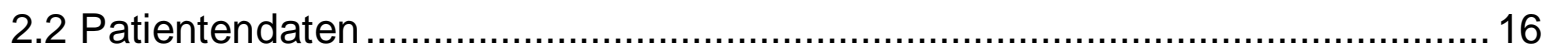

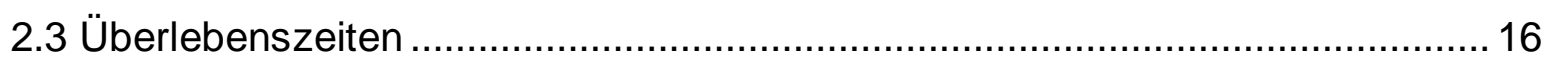

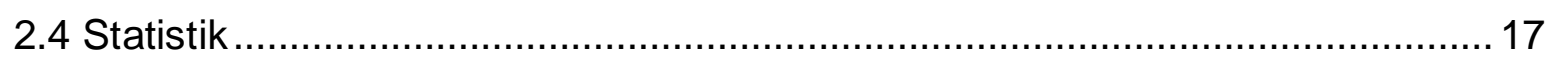

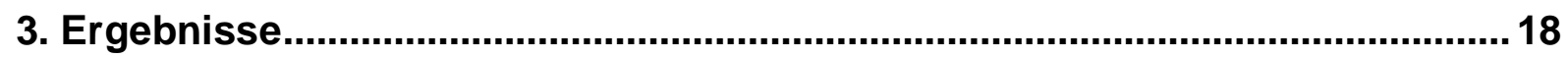

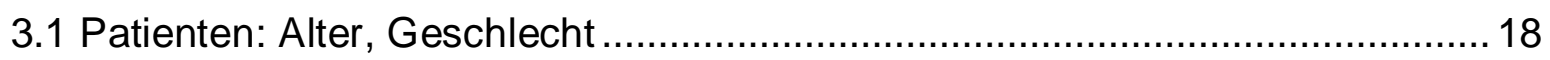

3.2 Symptome und Begleiterkrankungen ...................................................... 19

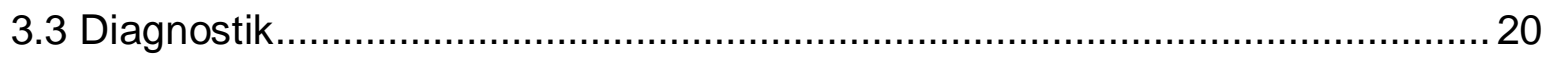

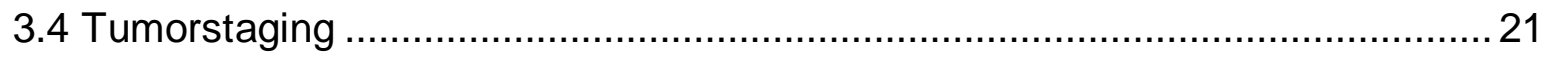

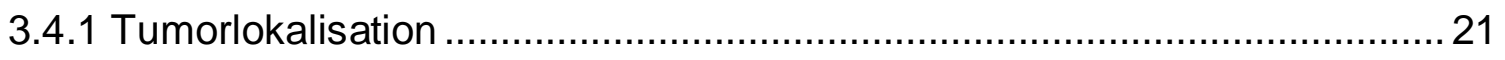

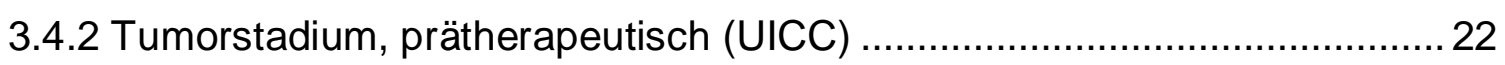

3.4.3 Präoperatives Tumorstadium in Gruppe 1 ........................................... 22

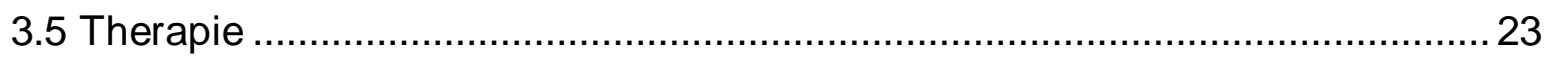

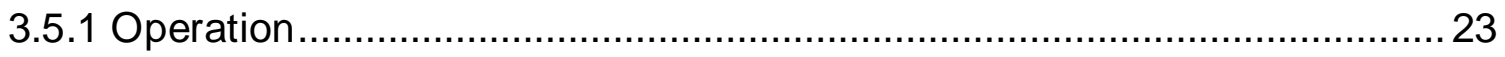

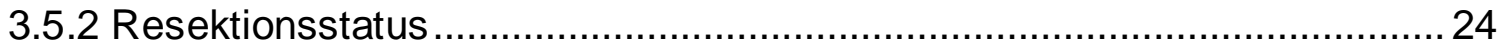

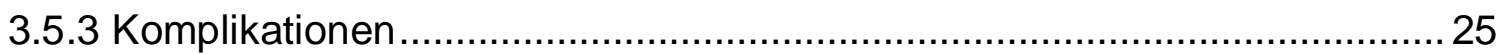

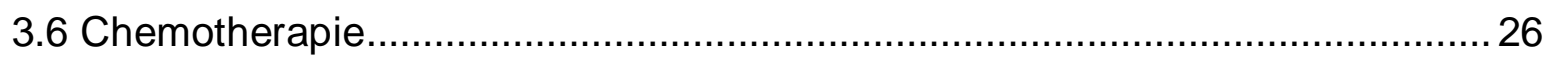

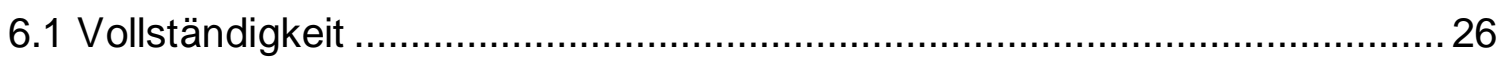

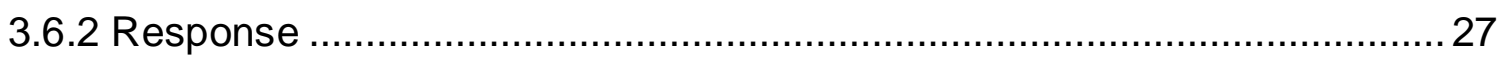

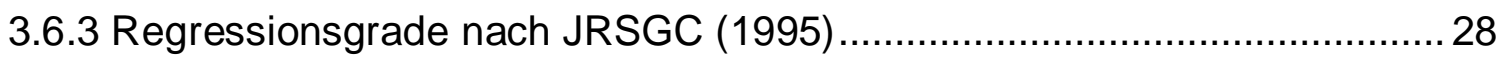

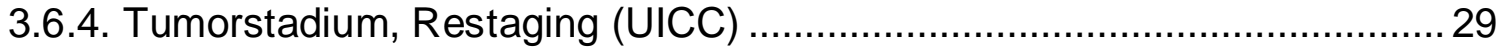

3.6.5 Tumorstadium, postoperativ (UICC) ................................................ 30

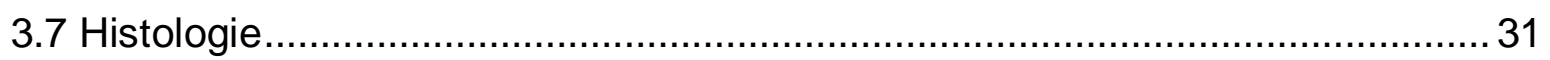

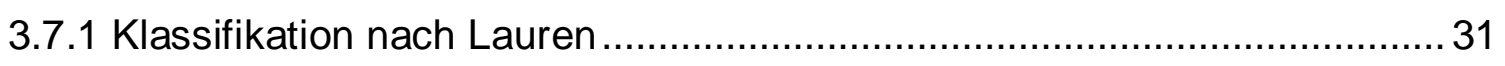

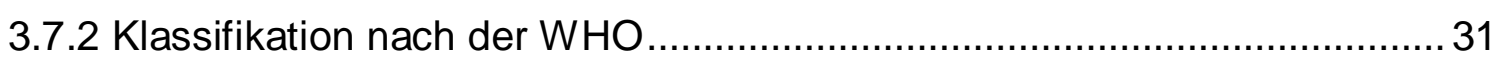

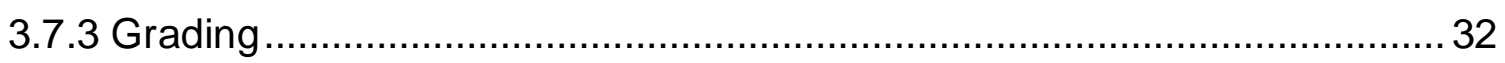

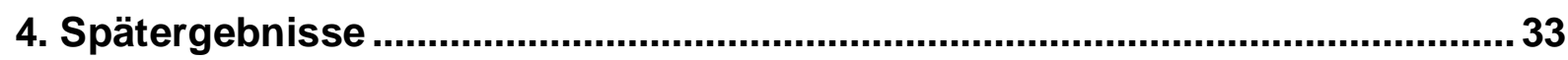




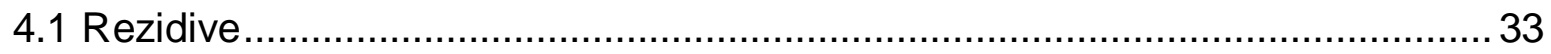

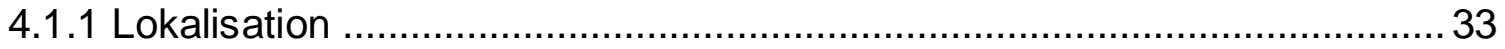

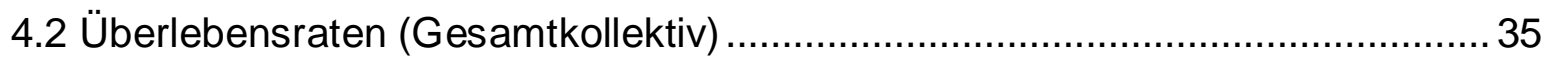

4.2.1 Tumorstadium (UICC), prätherapeutisch ................................................ 35

4.2.2. Tumorstadium (UICC), postoperativ ...................................................... 36

4.2.3. Tumorstadium (UICC I/II und III/IV), prätherapeutisch ............................. 37

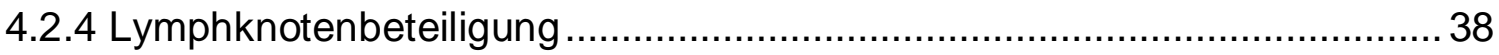

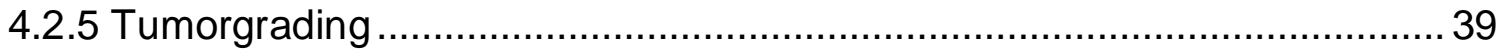

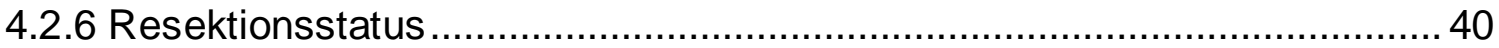

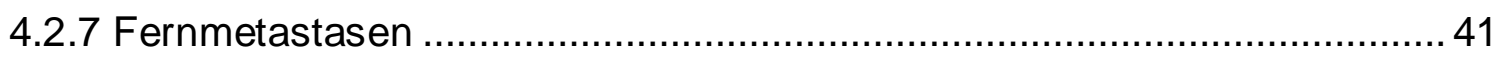

4.2.8 R0-/R+-Resektion und Fernmetastasen ............................................ 42

4.2.9 Chemotherapie vs. nur Operation ....................................................... 43

4.2.10 Responder versus Nicht-Responder versus nur Operierte ..................... 44

4.2.11 Tumorstadium (UICC), prätherapeutisch ............................................ 45

4.2.12 Tumorstadium (UICC), postoperativ ..................................................... 47

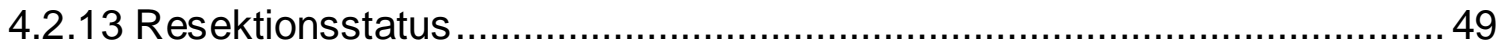

4.2.14 Resektionsstatus und Fernmetastasen.............................................. 51

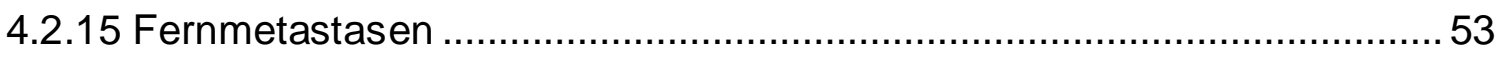

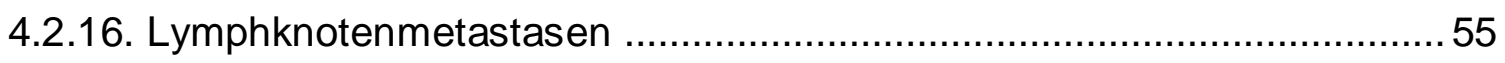

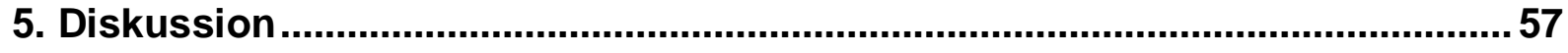

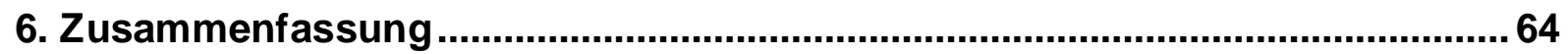

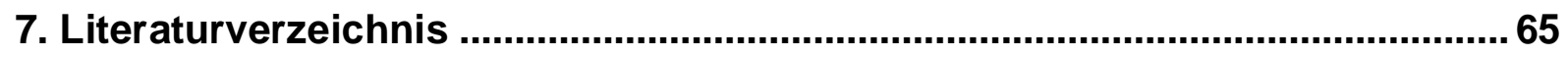

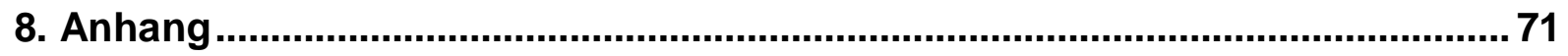

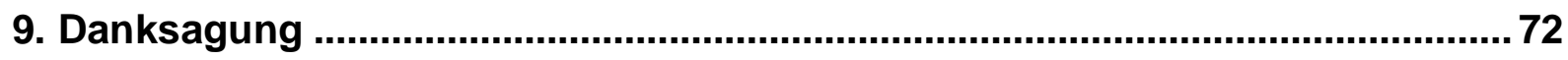




\section{Einleitung}

\subsection{Aktuelle Situation und Literaturübersicht}

Das Magenkarzinom ist ein globales Problem. Es ist der zweithäufigste diagnostizierte Krebs weltweit, in Deutschland die sechsthäufigste tumorbedingte Todesursache bei Männern und die vierthäufigste bei Frauen [31]. 12\% aller Malignom-abhängigen Todesfälle sind auf das Magenkarzinom zurückzuführen [33,57]. Das Auftreten variiert: epidemiologischen Untersuchungen zufolge sinkt die Inzidenz des Magenkarzinoms in Ländern wie USA, Europa und Australasien; in China, Japan, Südamerika, Osteuropa und im mittlerem Osten hingegen bleibt die Inzidenz hoch (Japan: ca. 90/100.000 Einwohner pro Jahr). In diesen Staaten tritt das Magenkarzinom rund vier- bis sechsmal häufiger auf als im Vergleich zu den westlichen Ländern [31].

Die Mortalität ist unverändert hoch. Am Magenkarzinom sterben in Deutschland nach Angaben des Robert-Koch-Instituts 13.000 Menschen pro Jahr, in den USA sind es 13.600 Menschen [33].

Die einzige kurative Therapieoption stellt die Operation mit kompletter Tumorresektion (R0) dar. Doch das Karzinom wird bei über der Hälfte der Patienten im fortgeschrittenem Stadium (UICC III/IV) diagnostiziert und die Chance auf eine vollständige Entfernung des Tumors sinkt. Von diesen Patienten werden weniger als $60 \%$ kurativ reseziert [19].

Die Prognose ist in frühen Stadien günstig, bei fortgeschrittenem Tumor schlecht. Die 5-Jahres-Überlebensrate (5-JÜR) liegt bei 40-50\% in den westlichen Ländern und Japan $[14,19,35,59]$. Unter kurativer Therapieintention haben frühe Stadien, UICC la und Ib, eine 5-JÜR von $83 \%$ und $69 \%$ sowie $43 \%$ im UICC-Stadium II; bei fortgeschrittenen Tumorstadien sinkt sie erheblich und liegt zwischen 28\% (UICC III) und 8,7\% (UICC IV) [19,33,56,57]. Der Frühdiagnose des Magenkarzinoms kommt daher eine zentrale Bedeutung zu. 
Das typische Erkrankungsalter des Magenkarzinoms liegt zwischen dem 40. und 70. Lebensjahr, der Altersgipfel im 65. Lebensjahr; mit steigendem Alter nimmt die Inzidenz zu. Männer sind mit einem Verhältnis von 1,7:1 etwas häufiger betroffen als Frauen [28].

Als ätiopathogenetisch relevant gilt vor allem die Kumulation mehrerer Faktoren, die zur Entstehung von Gewebsdysplasien mit hohem Entartungsrisiko führen. So können genetische Faktoren zusammen mit exogenen Faktoren wie Lifestyle und Ernährung die Entstehung begünstigen [27].

Eine positive Familienanamnese (familiäres Magenkarzinom) und auch die Zugehörigkeit zu bestimmten ethnischen Gruppen, wie Hispanic Americans, Native Americans und Afroamerikanern gehören zu den Risikofaktoren [27,28]. Weiterhin sind hohe Salzzufuhr, Verzehr von gepökeltem Fleisch (Nitrosamine), ballaststoffarme Ernährung, Nikotin- und exzessiver Alkoholkonsum mit einem hohen Magenkarzinomrisiko verbunden [27,54]. Die Helicobacter-pylori-Infektion wird von der WHO als Karzinogen der Gruppe I angesehen.

Eine multifaktorielle Ätiopathogenese gilt als gesichert [27,28,54].

In der Folge entstehen typische Gewebeveränderungen wie Morbus Menetrier und rezidivierende Ulcera ventriculi, die mit einem hohen Entartungsrisiko verbunden werden oder bereits Karzinomvorstufen darstellen. Über 50\% der Magenkarzinome haben ihren Ursprung in einer intestinalen Metaplasie, die im Rahmen einer chronisch-atrophischen Gastritis Typ B durch die Umwandlung der Magenschleimhaut in differenziertes intestinales Epithel (Becherzellen, resorbierende Zellen mit Mikrovillisaum, PANETH-Körnerzellen u.a.) entstehen kann [60].

Weiterhin werden Patienten mit subtotaler Magenresektion (insbesondere Billroth I), Vagotomie oder Gastroenterostoma zur Risikogruppe gezählt; durch das Fehlen des Pylorus kann es zu einem Gallereflux und damit zu einer ständigen Reizung der Schleimhaut ähnlich wie bei einer chronischen Gastritis kommen; nachgewiesen ist dies jedoch nicht $[28,57]$.

Neben der multifaktoriellen Ätiopathogenese bestehen beim Magenkarzinom unspezifische klinische Symptome wie Übelkeit, Dyspepsie, Erbrechen, Völlegefühl oder Dysphagie, insbesondere eine Abneigung gegen Fleisch. Die Unterscheidung 
zur klinischen Symptomatik einer Gastritis, eines peptischen Ulkus oder einer RefluxÖsophagitis ist kaum möglich. Die Folge ist, dass das Magenkarzinom in über $50 \%$ der Fälle erst in fortgeschrittenem Stadium (UICC III-IV) diagnostiziert wird. Diagnosen im Frühstadium sind in den westlichen Ländern im Gegensatz zu Japan Zufallsbefunde bei einer Gastroskopie [2,19,57]. Meist werden die Patienten erst beim Auftreten von Spätsymptomen wie Gewichtsverlust/Kachexie, Hämatemesis und/oder Teerstuhl gastroskopiert: ein bereits palpabler Oberbauchtumor Hepatomegalie, Aszites und Virchow-Lymphknoten können als Zeichen eines fortgeschrittenen Erkrankungsstadiums und der Metastasierung auftreten [28,54,57].

In der Diagnostik hat die Endoskopie eine zentrale Bedeutung. Neben der Beurteilung von Lokalisation und Ausdehnung ist nur durch die Histologie im Rahmen der Biopsie eine eindeutige Diagnose möglich. Ca. $5 \%$ der malignen Ulcera ventriculi imponieren endoskopisch primär benigne, sodass alle Ulzera zur histologischen Dignitätsklärung mehrfach (5-10mal) biopsiert werden sollten [54,57].

Bildgebende Verfahren wie Abdomen-Sonographie, Endosonographie (EUS), CTAbdomen und die diagnostische Laparoskopie werden zum Staging durchgeführt; sie liefern wichtige Informationen über die lokale Tumorausbreitung und über das systemische Erkrankungsstadium wie den Lymphknotenbefall und die Fernmetastasierung [28]. Tumormarker, wie Ca19-9, CEA und Ca72-4 sind wichtige Verlaufsparamater besonders in der Nachsorge, sie dienen nicht der Primärdiagnostik [2,28].

Nur die frühzeitige Diagnose kann zu einer Verbesserung der Prognose des Magenkarzinoms führen. Durch regelmäßige Screeninguntersuchungen, wie sie in Japan praktiziert werden, wurden bei den Patienten weniger häufig fortgeschrittene Karzinome diagnostiziert und die karzinombedingte Mortalität der gescreenten Patienten war nur etwa halb so hoch wie ohne Screening [30]. Infolge dieses Screening-Programms konnte in Japan eine Resektionsrate von $90 \%$ erreicht werden. In Ländern ohne Screening liegt die Resektionsrate bei 50\% [54]. In Deutschland ist dieses Verfahren nicht etabliert [57]. 
Im Rahmen der Diagnostik und des Stagings werden der Lokalisation sowie der histologischen Klassifikation nach WHO und Lauren besondere Bedeutung beigemessen; diese Charakteristika des Magenkarzinoms sind entscheidend für das chirurgische Vorgehen und haben prognostische Bedeutung.

Es werden proximale (10\%), in der Cardia- und Fundusregion gelegene Karzinome, von den distalen, im Corpus oder Antrum $(>86 \%$,) unterschieden. Proximale Karzinome neigen bereits früh und häufig zur Metastasierung und haben daher eine schlechtere Prognose. Distale Karzinome sind für die Prognose wegen der späten Metastasierung günstiger einzustufen [60].

Die pathologisch-anatomische Einteilung des Magenkarzinoms erfolgt nach der Klassifikation der WHO und reicht vom Adenokarzinom hoher Differenzierung bis zum undifferenzierten Karzinom. Eine Sonderform stellt das Siegelringkarzinom mit dem höchsten Metastasierungsgrad dar [60]. Karzinome mit einer hohen Differenzierung weisen eine bessere Prognose auf als weniger gut differenzierte Tumoren, die einen hohen Malignitätsgrad haben.

Die histologische Klassifikation nach Lauren ist für das chirurgische Vorgehen entscheidend und die drei Typen nach Lauren haben aufgrund ihres Wachstumsund Metastasierungsmusters eine unterschiedliche Prognose. Der intestinale Typ (50\%) zeigt ein lokalisiertes, gut begrenztes Wachstum, metastasiert spät und ist deshalb prognostisch günstiger. Im Gegensatz dazu imponiert der diffuse Typ (40\%) durch infiltrierendes Wachstum und eine frühe Metastasierung, weshalb seine Prognose weniger gut einzuschätzen ist. Aufgrund des unterschiedlich invasiven Wachstums müssen der diffuse und der intestinale Typ mit dementsprechend weiten Sicherheitsabständen reseziert werden: Intestinal: $>5 \mathrm{~cm}$, diffus: $>8 \mathrm{~cm}$ [24].

Bei Mischformen (5-10\%) erfolgt die Einteilung histologisch nach dem Anteil mit höchstem Malignitätsgrad. 
Im Rahmen der diagnostischen Verfahren und des Stagings wird das Magenkarzinom nach der TNM- und UICC-Klassifikation eingeteilt.

Die TNM-Einteilung beschreibt die lokale und systemische Ausbreitung des Magenkarzinoms. Die Kenntnis über die lokale Infiltrationstiefe $(T)$ und über die Ausdehnung der lymphogenen Metastasierung $(N)$ hat sowohl für das Ausmaß des chirurgischen Vorgehens als auch für die Prognose eine Bedeutung.

Die Tumorausbreitung wird entweder klinisch, cTNM (c=clinical) meist prätherapeutisch, und/oder chirurgisch, pTNM ( $p=$ histopathologisch) postoperativ, eruiert.

\section{Tabelle 1 TNM-Klassifikation}

\section{T Primärtumor}

T1m Lamina propria, Mucosa

T1sm Submukosa

T2a Muscularis prop.

T2b Subserosa

T3 Penetration der Serosa

T4 Infiltration der Nachbarstrukturen (1)

Anmerkung: (1) Nachbarstrukturen des Magens: Milz, Colon transversum, Leber, Zwerchfell, Pankreas, Bauchwand, Nebenniere, Niere, Dünndarm, Retroperitoneum

\section{N Regionäre Lymphknoten}

$\mathrm{Nx}$ regionäre Lymphknoten nicht beurteilbar

N1 1-6 Lymphknoten

N2 7-15 Lymphknoten

N3 >15 Lymphknoten

\section{Fernmetastasen}

Mx Fernmetastasen nicht beurteilbar

MO keine Fernmetastasen

M1 Fernmetastasen 
Die unterschiedlichen Tumorstadien werden zudem nach der „Union Internationale Contre le Cancer" (UICC) bzw. nach der "World Health Organization“ (WHO) eingeteilt $[12,13]$.

Diese Stadieneinteilung ist von besonderer Bedeutung für die Prognose und die Therapieplanung von Patienten mit Magenkarzinom. Sie fasst die unterschiedliche prognostische Bedeutung der lokalen und systemischen Ausbreitung (TNMKlassifikation, s.o.) des Magenkarzinoms in übergeordneten Stadien zusammen.

Tabelle 2 Stadieneinteilung nach UICC

$\begin{array}{llll}\text { Stadium 0 } & \text { Tis } & \text { N0 } & \text { M0 } \\ \text { Stadium I A } & \text { T1 } & \text { N0 } & \text { M0 } \\ \text { Stadium I B } & \text { T1 } & \text { N1 } & \text { M0 } \\ & \text { T2a/b } & \text { N0 } & \text { M0 }\end{array}$

$\begin{array}{llll}\text { Stadium II } & \text { T1 } & \text { N2 } & \text { M0 } \\ \text { T2a/b } & \text { N1 } & \text { M0 } \\ \text { T3 } & \text { N0 } & \text { M0 }\end{array}$

$\begin{array}{lll}\text { Stadium III A } & \text { T2a/b N2 } & \text { M0 }\end{array}$

$\begin{array}{llll}\text { T3 } & \text { N1 } & \text { M0 } \\ \text { T4 } & \text { N0 } & \text { M0 }\end{array}$

$\begin{array}{llll}\text { Stadium III B } & \text { T3 } & \text { N2 } & \text { M0 }\end{array}$

Stadium IV T4 N1,N2,N3 M0

$\mathrm{T} 1, \mathrm{~T} 2, \mathrm{~T} 3 \quad \mathrm{~N} 3 \quad \mathrm{M} 0$

Jedes $T \quad$ jedes $N \quad M$ 
Die einzige kurative Therapie beim Magenkarzinom stell die Operation nach chirurgisch-onkologischen Kriterien dar. Je nach Tumorgröße, Lokalisation und histologischem Typ nach Lauren erfolgt die totale oder erweiterte Gastrektomie oder bei distaler Lokalisation und kleinen Tumoren die subtotale Gastrektomie (Billroth II Resektion). Im Rahmen der Gastrektomie wird eine systematische Lymphadenektomie durchgeführt; standardmäßig werden in den westlichen Ländern Kompartment 1 und 2 (D1 und D2, s.u.) reseziert. In Japan wird die D3Lymphadenektomie häufiger durchgeführt $[51,60]$.

Eine Kartographie der Lymphknotenstationen des Magens wurde 1993 von Maruyama erstellt. Es werden drei Kompartimente unterschieden:

I perigastrale Lymphknoten paracardial, entlang der kleinen und großen Kurvatur, supra- und infrapylorisch (LN 1-6) (D1-Kompartment)

II Lymphknoten des Ligamentum hepatoduodenale, A.hepatica communis, Truncus coeliacus, A.lienalis (+Milzhilus) $\quad$ (LN7-11) $\quad$ (D2-Kompartment)

III paraaortale, retroperitoneale, mediastinale Lymphknoten und im Bereich der Mesenterialgefäße (LN12-14) (D3-Kompartment)

Das D1-Kompartment ist schon bei den frühen Stadien zu über $80 \%$ befallen, bei fortgeschrittenen Tumoren ist sehr häufig auch das D2-, evtl. D3 -Kompartment, betroffen $[19,51,54,60]$.

Bei der Resektion des D2-Kompartments erfolgt die Entfernung der Lymphknoten der kleinen Kurvatur (Kompartment I) en bloc mit dem Magen und den Lymphknoten von Ligamentum hepatoduodenale, Pankreasoberrand und Milzhilus (Kompartment II).

Die Lymphknotenstation 13 hinter dem Duodenum muss als Grenzlymphknoten zum Kompartment III (retropankreatisch, mesenterial) mit entfernt werden. Sein Befall zeigt, dass Kompartment 3 befallen ist und keine R0-Resektion mehr erreicht werden kann. Auch Omentum minus et majus werden entfernt. Die Sicherheitsabstände werden in Abhängigkeit vom Lauren Typ festgelegt [2,31,51].

Die T1-Stadien (T1m/T1sm) und das Magenfrühkarzinom (Tis) mit einem Lymphknotenbefall $<10 \%$ können endoskopisch reseziert werden, sofern eine R0Resektion garantiert werden kann (5-JÜR 99\%). Sollte dies nicht der Fall sein, werden sie wie fortgeschrittene Stadien behandelt $[2,18,19,29,40]$. 
Unter kurativer Therapieintention ist die R0-Resektion entscheidend. Bei Residualtumor R1, mikroskopisch Tumorrest am Resektionsrand, oder R2, makroskopisch Tumorrest am Resektionsrand, wird die 5-Jahresgrenze nicht erreicht [19,33]. Ob eine R0-Resektion gelingt, hängt vom Ausmaß der lymphogenen Metastasierung, die früh erfolgt, ab. Besonders die intramurale Infiltration $(T)$ ist ein wichtiges Charakteristikum, um die Wahrscheinlichkeit der Lymphknotenbeteiligung ungefähr abschätzen zu können. In Abhängigkeit vom T-Stadium (Infiltrationstiefe des Primärtumors) steigt das Risiko für Lymphknotenmetastasen rapide an. Denn auch bei noch auf die Organwand beschränkten Tumoren ist bereits mit Lymphknotenmetastasen zu rechnen; bereits $23 \%$ der auf die Submukosa beschränkten Stadien (Tsm) haben Lymphknotenmetastasen und ab T2, einer Infiltrationstiefe bis in die Muscularis, finden sich bei bis zu $66 \%$ positive Lymphknoten. Besteht eine Infiltration bis zur Serosa (T3) oder darüber hinaus (T4) haben nahezu alle Patienten ( $86 \%$ bis $>90 \%$ ) Lymphknotenmetastasen. Deshalb wird bereits ab T2 eine radikale Lymphadenektomie, D2-Dissektion (s.u.), empfohlen $[19,22,36,32,54]$.

Auch für die Prognose ist der Lymphknotenbefall der wichtigste Faktor: bei N0 liegt die 5 -JÜR bei $88 \%$, bei N3 liegt sie bei $26 \%$. Die Lymphadenektomie des D2Kompartments hat gegenüber der D1-Lymphadenektomie einen Überlebensvorteil von $64 \%$ zu $31 \%$ gezeigt $[19,38,43,50]$. Das Ausmaß der Lymphknotendissektion stellt neben der T- und N-Klassifikation einen unabhängigen Prognosefaktor dar [15].

10-30\% der Magenkarzinome haben bereits bei Erstdiagnose Fernmetastasen $[19,31,32,45]$. Sie finden sich insbesondere im Peritoneum, auf hämatogenem Weg relativ spät und primär in der Leber. Eine Metastasierung per continuitatem erfolgt in erster Linie in den Ösophagus, ins Duodenum, Kolon und das Pankreas $[19,51,54,60]$.

Doch auch nach kurativer Resektion bleibt die Prognose bei den fortgeschrittenen UICC-Stadien ungünstig [14]. Haben die Stadien I mit einer 5-JÜR von 83\% (la) bis $69 \%$ (Ib) noch eine eher gute Prognose, liegt die 5-JÜR ab Stadium II bei unter 50\% (43,75\%). Im Stadium III erreichen lediglich $28,6 \%$ (IIla) und $17,7 \%$ (IIllb) die 5Jahresgrenze und im Stadium IV sind es weniger als $10 \%$ der Patienten $(8,7 \%)$. 
Postuliert wird, dass durch die Operation die im peripheren Blut zirkulierenden und die im Knochenmark nachweisbaren Tumorzellen, Mikrometastasen, nicht behandelt werden können [14]. So wird postoperativ die Prognose durch das Auftreten eines Rezidivs bestimmt; dies stell ein weiteres Problem in der Therapie des Magenkarzinoms dar. Denn auch nach R0-Resektion und D2-Lymphadenektomie liegt die Rezidivrate bei 69\% [28,31]. Mit 87\% tritt das lokoregionäre Rezidiv am häufigsten auf. Aber auch Rezidive in Form von Peritonealkarzinose und Lebermetastasen sind ein signifikantes Problem [19,38,49].

Durch zur Operation zusätzliche, adjuvante, Therapiemodalitäten wie Radiatio und Chemotherapie sollte die lokale Tumorkontrolle und die Therapie von intraoperativ disseminierten Tumorzellen verbessert werden; dadurch wollte man die Rezidivrate senken und die Prognose verbessern [14,25,31].

Externe und intraoperative Bestrahlung (IORT) wurden eingesetzt [14]. Die externe Bestrahlung erwies sich bei der Verringerung von Lokalrezidiven als ineffizient [14]. Die IORT verbesserte die lokale Tumorkontrolle, Rezidive in Form von Fernmetastasen (19,7\%) und/oder peritonealer Aussaat (31,8\%) konnte sie jedoch nicht verhindern. Das Gesamtüberleben konnte nach IORT mit einer 5-JÜR von 58\% gegenüber den nur Operierten mit 59\% nicht verlängert werden [14].

Postoperative Bestrahlung verbesserte die lokale Tumorkontrolle bei fortgeschrittenen Tumoren (T3 bzw. $\mathrm{N}_{+}$); doch das Gesamtüberleben konnte mit einer 5-JÜR von 12\% nach adjuvanter Bestrahlung nicht verlängert werden, bei den nur Operierten lag die 5-JÜR bei 20\% [25,54].

Adjuvante Radiochemotherapie verbesserte bei fortgeschrittenen Stadien (II-IV) die lokale Tumorkontrolle und nach 3 Jahren war das Gesamtüberleben mit $50 \%$ versus $41 \%$ und das mediane Überleben von 36 Monaten gegenüber 27 Monaten nach Radiochemotherapie signifikant besser $[34,27]$. Jeodch entsprach das chirurgische Vorgehen in diesen Studien nicht europäischen Standards; so wurden Patienten mit unzureichender Resektion (R1), mit T2-3 - Tumoren ohne Lymphknotendissektion oder T3-Tumoren mit D1-Dissektion adjuvant bestrahlt und chemotherapiert.

Eine italienische Studie beobachtete für mit kurativer Intention operierte Magenkarzinome im Stadium III und IV nach Radiochemotherapie (FOLFOX-4) eine Überlebensrate von 50,1\% nach 3 Jahren [44]. Bei lokal begrenzten Tumoren konnte kein Überlebensvorteil erreicht werden [55]. 
Neben der kombinierten Radiochemotherapie wurde die alleinige adjuvante Chemotherapie mit demselben Ziel, Reduktion der Rezidivrate und Verbesserung des Gesamtüberlebens, untersucht [31]. Das progressionsfreie Überleben konnte verbessert werden, jedoch nicht die aktuelle mit alleiniger Chirurgie (D2-Dissektion) erreichte 5-JÜR von ca. 50\%. Multiple Chemotherapie-Schemata wie FAM (5FU/Doxorubicin/Mitomycin), FAMTX (5-FU/Docorubicin/Metothrexat/ Leukovorin) oder ECF (Epirubucin/Cisplatin/5-FU) wurden untersucht; die 5-JÜR lagen bei 30\%, $43 \%$ oder $36 \%$ bei Responseraten von $10-40 \%[11,31,33,41]$. Kombinationen mit Cisplatin zeigten Responseraten bis zu über $50 \%$, mussten aber aufgrund sehr viel höhere Toxizität, insbesondere Neutropenie, abgebrochen werden [31]. Eine japanische Studie beobachtete ein verlängertes rezidivfreies Überleben für Tumoren im Stadium II/III nach D2-Dissektion nach der Gabe von S-1, einem Fluorpyrimidinanalogon; das Follow-up betrug 3 Jahre, hierbei lag das Gesamtüberleben bei $80,1 \%$ und das rezidivfreie Überleben nach 3 Jahren bei $72,2 \%$ versus $59,6 \%$ in der Kontrollgruppe [50]. In Subgruppen (T2 N1) wurde nach der adjuvanten Gabe von Uracil-Tegafur von einem verlängerten rezidivfreien Überleben berichtet; das Gesamtüberleben lag nach 5 Jahren bei 86\% versus 73\% in der Kontrollgruppe [38]. Tegafur wird über das Cytochrom-P-450 verstoffwechselt; Polymorphismen in den für dieses Enzymsystem kodierenden Genen bewirken eine erhöhte Toxizität bei europäischen Patienten, sodass sich die japanischen Studiendaten nicht ohne weiteres auf europäische Patienten übertragen lassen und umfangreiche Studien erforderlich werden [27].

Ein weiterer Therapieansatz besteht in der Gabe von Antikörpern wie Cetuximab gegen EGFR und Bevacizumab gegen VEGF. Die Überexpression des EGFR ,epidermal growth factor resceptor' und des VEGF, vascular endothlial growth factor soll beim Magenkarzinom mit einer schlechten Prognose korrelieren. Bekannt ist bisher, dass diese Antikörper Ansprechraten von 44\% und 65\% möglich machten. Langzeitergebnisse über derartige Therapiekonzepte stehen jedoch noch aus [27].

Insgesamt konnte mit adjuvanten Therapien keine Verbesserung der Prognose erzielt werden, die 5-JÜR liegt unverändert bei $50 \%$. 
Da durch intraoperative Bestrahlung (IORT) eine Verbesserung der lokalen Tumorkontrolle erreicht werden konnte, wurde eine neoadjuvante Chemotherapie vorgeschaltet [14]. Das Ziel war eine Verlängerung des gesamten und krankheitsfreien Überlebens. Durch präoperative Devitalisierung und Verkleinerung des Primärtumors wollte man lokale R0-Resektabilität erreichen, die Ausbreitung von Tumorzellen insbesondere in Lymphknoten frühzeitig systemisch therapieren und die intraoperative Tumorzellaussaat senken [21,39,54]. Das Erreichen eines niedrigeren UICC-Tumorstadiums, Downstaging, sollte die R0-Resektionsrate besonders auch von fortgeschrittenen Tumorstadien (UICC lb-III) erhöhen und zusammen mit einer frühzeitigen systemischen Therapie von Mikrometastasen zu einer Reduktion von Lokalrezidiven und Fernmetastasen führen und infolge die Prognose verbessern $[19,21,39]$. Verschiedene neoadjuvante Chemotherapie-Schemata wurden untersucht: PELF (Cisplatin/Epirubicin/Leukovorin/5-FU), PELF plus Docetaxel, FLEP (5-FU/Leukovorin/Cisplatin/Etoposid) [4,21,39]. Die R0-Resektionsraten lagen unverändert zwischen $47 \%$ und $50 \%$, bei Ansprechraten von 36,8\%, 50\% und $70 \%$ $[21,39,41]$. Bei einer scheinbaren Tendenz zur Reduktion des lokalen Rezidivs konnte das Gesamtüberleben jedoch nicht verbessert werden. Es lag unverändert bei $50 \%[21,39]$.

Es zeigte sich, dass nur Patienten mit histopathologischem (objektivem) Tumoransprechen von einer neoadjuvanten Chemotherapie profitierten und auch dann war das Gesamtüberleben nicht verbessert worden. Für alle anderen Patienten beinhaltete das Vorgehen keinerlei Überlebensvorteil. [19,42,59]. Da es derzeit keine sicheren Prädiktoren über das Ansprechen gibt, sollte bei Patienten mit ungeeigneten Tumorstadien (UICC $\mathrm{I} / \mathrm{II}$ oder $\mathrm{M}+$ ), bei denen sich eine geringe Wahrscheinlichkeit eines Ansprechens gezeigt hat, von einer neoadjuvanten Chemotherapie abgesehen werden; in diesem Zusammenhang sind eine Peritonealkarzinose und/oder Lebermetastasen auszuschließen [10,19,21,40]. Die häufigsten Nebenwirkungen waren Knochenmarksuppression mit Neutropenie und Thrombozytopenie, Übelkeit und Erbrechen, Stomatitis und Diarrhoe [21,27,39]. Cisplatin in Kombination mit Substanzen wie Irinotecan als Topoisomeraseinhibitor oder Docetaxel und 5-FU oder S-1 sind Gegenstand aktueller Studien, jedoch aufgrund zum Teil hoher Toxizität problematisch in der Durchführung [62,63].

Mit den bisherigen neoadjuvanten Therapien konnte keine Verbesserung der aktuellen Prognose von ca. 50\% erreicht werden. 


\subsection{Thematische Problematik}

In einer Studie zur palliativen Therapie beim Magenkarzinom konnte mit der Kombination von Cisplatin, Epirubicin, Leukovorin, 5-FU (PELF) eine signifikante Verbesserung der medianen Überlebenszeit erreicht werden; die Responserate lag bei 40\% [19]. Aufgrund dieser Ergebnisse wurde in einer Pilotstudie der Einfluss auf die Prognose bzw. auf die Rezidivhäufigkeit einer PELF-Chemotherapie als neoadjuvantes Setting geprüft.

Jedoch waren die R0-Resektionsraten und Überlebensraten in der Studie nicht höher als sie auch nach primärer Resektion bei diesem Patientengut zu erwarten waren und die objektive Analyse der Überlebenszeiten war aufgrund unzureichender prätherapeutischer Staging-Untersuchungen und Schwierigkeiten bei der Messung der Tumormasse nur eingeschränkt möglich [19].

Das klassische Schema des PELF-Protokolls, wie es auch in der vorliegenden Studie durchgeführt wurde, sieht vor, dass den Patienten in 2 Zyklen à 6 Wochen die Substanzen Cisplatin, Epirubicin, Leucovorin und 5 - Fluoruracil verabreicht werden.

Cisplatin (cis-Diamindichloroplatin) ist ein anorganischer Schwermetallkomplex und gehört zur Gruppe der Platin-Komplex-Bildner. Es interagiert mit der DNA und führt zu Quervernetzungen (cross-linking) innerhalb und zwischen den DNA-Strängen und somit zur Hemmung der DNA-Replikation und -Transkription. Seine Zytotoxizität beruht auf der resultierenden Hemmung der Zellteilung in der G2-Phase des Zellzyklus.

Epirubicin ist ein Antracyclin. Es bindet an die Topoisomerase lla und hemmt so die Zellteilung.

Leukovorin (Calziumfolinat) ist der aktive Metabolit der Folsäure. In Kombination mit 5-FU erhöht es dessen Zytotoxizität. 
Das Antimetabolit 5 - Fluoruracil weist eine strukturelle Ähnlichkeit mit den Pyrimidinbasen Cytosin, Thymin und Uracil auf. Bei der Zellteilung wird es anstatt dieser in die DNA eingebaut und es resultiert die Hemmung des Zellwachstums.

Darüber hinaus blockiert 5-FU die Thymidylatsynthase und somit die Umwandlung von Desoxyuridylsäure (dUMP) in den DNA-Baustein Thymidylat (dTMP). Dies führt ebenfalls zu einer Hemmung des Zellwachstums.

Insgesamt wurden in den letzten 15 Jahren die medianen Überlebenszeiten in Subgruppen verbessert, eine Verlängerung des Gesamtüberlebens konnte jedoch nicht erzielt werden. Dieses liegt unverändert mit Berücksichtigung aller Tumorstadien bei $50 \%[14,19,35,42,59]$.

Die Prognose beim Magenkarzinom lässt sich nicht durch alleinige chirurgische Maßnahmen wie Erweiterung der Operation durch multiviszerale Resektionen verbessern. Nur die multimodalen Therapieansätze mit neoadjuvanter Therapie und Bestimmung der entsprechenden Indikationen lassen die systemische Ausbreitung in den Griff bekommen und die Tumorbiologie berücksichtigen [10,19,29,31,40,54]. 


\subsection{Fragestellung}

In der vorliegenden retrospektiven Pilotstudie wurde der Einfluss einer neoadjuvanten Chemotherapie nach dem PELF-Protokoll (Cisplatin, Epirubicin, Leucovorin und 5-Fluoruracil) auf die Prognose des fortgeschrittenen Magenkarzinoms, UICC Ib-III, geprüft.

Weiterhin wurden das Gesamtüberleben, die Rezidivhäufigkeit nach Chemotherapie, das rezidivfreie Überleben sowie die Auswirkungen der neoadjuvanten Chemotherapie auf die perioperative Morbidität und Mortalität untersucht. 


\section{Hauptteil}

\subsection{Material und Methode}

An der chirurgischen Universitätsklinik wurden im Rahmen der vorliegenden Studie im Zeitraum vom 01.01.2000 bis zum 31.12.2006 124 Patienten mit Magenkarzinom operiert.

Ein Teil dieser Patienten wurde nur operiert, der andere Teil der Patienten erhielt eine neoadjuvante Chemotherapie nach dem PELF-Protokoll. Die Zuteilung zu den Gruppen war nicht randomisiert.

Für die Durchführung der Chemotherapie mussten folgende Einschlusskriterien erfüllt sein: das Magenkarzinom wurde nach UICC in Stadium Ib - III eingeteilt, es war keine frühere Chemotherapie durchgeführt worden und es bestand keine andere Malignomerkrankung. Zudem durften weder die Leber- noch die Nierenfunktion eingeschränkt sein.

Ausschlusskriterien für eine neoadjuvante Chemotherapie waren eine Peritonealkarzinose, Lebermetastasen, ein frühes Tumorstadium (<T2N0, Typ I WHO) oder eine andere Tumorlokalisation (Ösophagus). 


\subsection{Patientendaten}

Die Daten aller 124 Patienten wurden für die vorliegende Studie retrospektiv ausgewertet. Dabei wurden 60 Patienten nicht berücksichtigt, da sie die Einschlusskriterien nicht erfüllt oder die Chemotherapie abgelehnt hatten.

In die vorliegende Studie wurden 64 Patienten aufgenommen und in 2 Gruppen eingeteilt:

Gruppe 1: 26 Patienten mit präoperativer Chemotherapie gemäß PELF-Protokoll Gruppe 2: 38 Patienten nur mit Operation

Zunächst wurden Gruppe 1 und 2 hinsichtlich Alters und Geschlechts, der Symptome, der Diagnostik, des Tumorstadiums, der Lokalisation und der Histologie, der Operation und des Resektionsstatus, der peritherapeutischen Komplikationen, des Auftretens von Rezidiven und ihres Überlebens miteinander verglichen.

In Gruppe 1 wurden entsprechend ihres Ansprechens auf die Chemotherapie Responder und Nicht-Responder unterschieden und hinsichtlich der Durchführung der Chemotherapie, der Tumorstadien, der Operation, des Resektionsstatus und des Überlebens verglichen.

\section{3 Überlebenszeiten}

Die Daten für das Überleben sind aus der Datenbank der Tumornachsorge des CCCF („Comprehensive Cancer Care Center Freiburg“) entnommen. Um Informationen über fehlende Daten hinsichtlich des Überlebens oder einer eventuellen adjuvanten Therapie, deren Nebenwirkungen und möglicher Rezidive zu erhalten, wurde ein Brief/Fragenkatalog an die Hausärzte verschickt.

Die Überlebensraten wurden zunächst im Gesamtkollektiv ( $n=64$ ), dann von Gruppe 1 und 2 analysiert. Anschließend wurden die Patienten zur Analyse der Überlebenszeiten in drei Gruppen unterteilt: Gruppe 1a Responder ( $n=20 / 64), 1 b$ Nicht-Responder ( $n=6 / 64)$ und Gruppe 2 nur Operierte ( $n=38 / 64)$. 


\subsection{Statistik}

Mit Hilfe des Programms Excel Version "Micros oft Office Enterprise 2007“ wurde eine Datenbank erstellt, in die die für das Magenkarzinom relevanten Daten aus den Krankenakten eingegeben wurden.

Die Überlebenszeiten wurden nach der Methode Kaplan und Meier berechnet. Als Ausgangspunkt zur Ermittlung der Überlebenszeit wurde das Operationsdatum gewählt. Als Endpunkt des Überlebenszeitraumes diente entweder das Todesdatum oder das Datum, zu dem der Patient zuletzt als lebend erfasst wurde; als Stichtag wurde der 31.10.2006 festgelegt.

Die mediane Überlebenszeit definierte sich durch den Zeitpunkt, an dem die kumulative Überlebenswahrscheinlichkeit 0,5 betrug.

Zur statistischen Beurteilung des Einflusses der klinischen und histologischen Faktoren auf die Überlebensprognose (Tumorklassifikation nach Lauren und WHO, Chemotherapie, Response, prae-/postoperatives T-Stadium, Lymphknotenstatus, Art der Resektion und Resektionsstatus, Rezidiv) wurde der Logrank-Test herangezogen. Das Signifikanzniveau lag bei $p=0,05$.

Als statistisches Programm für die Berechnung der Kaplan-Meier Kurven und der Signifikanz kam das SPSS IBM®SPSS $\circledast$ Statistics 15 zur Anwendung. 


\section{Ergebnisse}

\subsection{Patienten: Alter, Geschlecht}

Das Alter der Patienten in beiden Gruppen zusammen lag zwischen 38 und 91 Jahren, im Durchschnitt bei 67,86 Jahren (Median 69,00 Jahre).

Beim Vergleich der Altersverteilung unterschieden sich beide Gruppen nicht signifikant voneinander. Das Alter von Gruppe 1 lag im Median bei 64,5 Jahren (Range 38-76Jahre) bei einem Durchschnitt von 62,8 Jahren und das der Gruppe 2 im Median bei 73 Jahren (Range 43-91Jahre) bei einem Durchschnitt von 71,4 Jahren.

Tabelle 3 Altersverteilung in Gruppe $1+2$

\begin{tabular}{|l|c|c|}
\hline Alter & $\begin{array}{c}\text { Gruppe 1 } \\
\mathrm{n}\end{array}$ & $\begin{array}{c}\text { Gruppe2 } \\
\mathrm{n}\end{array}$ \\
\hline bis 69 & 21 & 13 \\
\hline 69/älter & 5 & 25 \\
\hline
\end{tabular}

Hinsichtlich der Geschlechterverteilung zeigte sich in beiden Gruppen ein Überwiegen der Männer. In Gruppe 1 lag das Verhältnis von Männern zu Frauen bei 3,33:1, in Gruppe 2 bei 1,53:1.

Gruppe 1 hatte 20 Männer und 6 Frauen, in Gruppe 2 waren 23 Männer und 15 Frauen. 


\subsection{Symptome und Begleiterkrankungen}

An klinischen Symptomen wurden in beiden Gruppen die typischen Spätsymptome des Magenkarzinoms in gleicher Häufigkeit beobachtet. Dysphagie, unspezifische epigastrische Beschwerden wie Druckgefühl, Magenschmerzen, postprandiale Oberbauchbeschwerden, Völlegefühl und Sodbrennen. Es waren zudem Hämatemesis/Magenblutung, Gewichtsabnahme und/oder Kachexie, thorakale und/oder retrosternale Beschwerden und Teerstuhl aufgetreten.

Tabelle 4 Verteilung der Symptome (Mehrfachnennungen)

\begin{tabular}{|l|c|c|}
\hline Symptome & Gruppe1 $(\mathrm{n}=26)$ & Gruppe 2 $(\mathrm{n}=38)$ \\
\hline Dysphagie & 8 & 10 \\
Epigastrische Beschwerden & 9 & 19 \\
Sodbrennen/Reflux & 5 & 5 \\
Hämatemesis/Magenblutung & 4 & 8 \\
Gewichtsverlust/Kachexie & 7 & 9 \\
\hline
\end{tabular}

In beiden Gruppen wiesen die Patienten dieselben Begleit- und Vorerkrankungen in ähnlicher Häufigkeit auf.

Tabelle 5 Begleiterkrankungen

\begin{tabular}{|l|c|c|}
\hline Begleiterkrankung & Gruppe1 $(\mathrm{n}=26)$ & Gruppe 2 $(\mathrm{n}=38)$ \\
\hline Chronische Gastritis & 7 & 12 \\
$\rightarrow$ (Z.n.) Ulcus ventriculi & 2 & 6 \\
Refluxösophagitis & 2 & 7 \\
Nikotinabusus & 5 & 6 \\
Hypertonie & 6 & 21 \\
Adipositas & 6 & 6 \\
\hline
\end{tabular}




\subsection{Diagnostik}

Zur Diagnosestellung und histologischen Sicherung des Karzinoms wurde bei allen Patienten als Standardmethode eine Ösophagogastroduodenoskopie (OEGDS) mit Entnahme multipler Biopsien durchgeführt.

Zum Tumorstaging wurden die Patienten folgendermaßen untersucht: das Ausmaß der Magenwandinfiltration und der lokoregionalen Lymphknotenbeteiligung (D1Kompartment) wurde bei nahezu allen Patienten ( $n=53 / 64)$ mit der Endosonographie (EUS) ermittelt.

Die systemische Ausbreitung in Lymphknoten des D2- und D3-Kompartments wie auch in Leber und Ovarien (Krukenberg-Tumor) wurde bei allen Patienten mittels CT und Abdomen-Sonographie eruiert.

Zum Ausschluss pulmonaler Fernmetastasen wurde ein Röntgen-Thorax angefertigt $(n=59 / 64)$. Einen Röntgen-Gastrografinschluck erhielten Patienten mit Schluckstörungen in der Anamnese ( $n=17 / 64)$.

Alle Patienten der Gruppe 1 und 11 der Gruppe 2 waren zum Ausschluss einer Peritonealkarzinose diagnostisch laparoskopiert worden.

Die Tumormarker CEA und CA19-9 wurden bei allen Patienten als Verlaufsparameter bestimmt.

Tabelle 6 Diagnostische Verfahren

\begin{tabular}{|l|c|c|}
\hline Methode & Gruppe1 $\quad(\mathrm{n}=26)$ & Gruppe2 $\quad(\mathrm{n}=38)$ \\
\hline OEGDS & 26 & 38 \\
EUS & 25 & 28 \\
CT & 26 & 38 \\
Laparoskopie & 26 & 11 \\
Sonographie-Abdomen & 9 & 18 \\
Röntgen-Thorax & 26 & 33 \\
Röntgen-Breischluck & 7 & 10 \\
\hline
\end{tabular}




\subsection{Tumorstaging}

\subsubsection{Tumorlokalisation}

Die Karzinome wurden in im Bereich des proximalen Magens gelegene und in eher weiter distal gelegene Tumoren unterschieden.

In der vorliegenden Studie waren die Karzinome hauptsächlich proximal lokalisiert ( $n=46 / 64)$ und traten in beiden Studiengruppen ähnlich häufig auf.

Distal gelegene Tumoren waren in beiden Gruppen insgesamt seltener vertreten ( $n=18 / 64)$ und kamen in beiden Gruppen ähnlich häufig vor.

\section{Tabelle 7 Tumorlokalisation}

\begin{tabular}{|c|c|c|}
\hline Lokalisation & $\begin{array}{c}\text { Gruppe 1 } \\
(\mathrm{n}=26)\end{array}$ & $\begin{array}{c}\text { Gruppe2 } \\
(\mathrm{n}=38)\end{array}$ \\
\hline Proximaler Magen & 20 & 26 \\
\hline Distaler Magen & 6 & 12 \\
$-\quad$ Corpus,Antrum/Pylorus & 3 & 7 \\
$-\quad$ Kurvatur & & \\
groß & 0 & 3 \\
klein & 3 & 2 \\
\hline
\end{tabular}




\subsubsection{Tumorstadium, prätherapeutisch (UICC)}

Beim Staging mittels Endoskopie und CT und bei der Auswertung der Daten wurde die Stadieneinteilung der "Union Internationale Contre le Cancer" (UICC) bzw. der „World Health Organization“ (WHO) berücksichtigt. Bei der Erhebung der Daten von Tabelle 8 wurden die mit CT und EUS ermittelten Tumorstadien zusammengefasst und es wurde jeweils die ungünstigere Diagnose berücksichtigt.

Die UICC-Stadien II und III waren zu Beginn der Therapie in beiden Gruppen am häufigsten diagnostiziert worden ( $n=52 / 64)$, Stadium lb bei 12 Patienten.

Tabelle 8 Tumorstadien prätherapeutisch (UICC)

\begin{tabular}{|l|c|c|}
\hline UICC & $\begin{array}{c}\text { Gruppe1 } \\
(\mathrm{n}=26)\end{array}$ & $\begin{array}{c}\text { Gruppe2 } \\
(\mathrm{n}=38)\end{array}$ \\
\hline $\mathrm{Ib}$ & 2 & 10 \\
\hline $\mathrm{II}$ & 7 & 13 \\
\hline $\mathrm{III}$ & 17 & 15 \\
\hline
\end{tabular}

\subsubsection{Präoperatives Tumorstadium in Gruppe 1}

Zu Beginn der Chemotherapie lagen bei den meisten Respondern und NichtRespondern fortgeschrittene Tumorstadien, UICC II $(n=7 / 26)$ und III $(n=17 / 26)$, in ähnlicher Häufigkeit vor. 2 Responder hatten Stadium Ib.

Tabelle 9 Tumorstadien prätherapeutisch (UICC)

\begin{tabular}{|l|c|c|}
\hline UICC & $\begin{array}{c}\text { Responder } \\
(\mathrm{n}=20)\end{array}$ & $\begin{array}{c}\text { Nicht-Responder } \\
(\mathrm{n}=6)\end{array}$ \\
\hline $\mathrm{Ib}$ & 2 & 0 \\
\hline $\mathrm{II}$ & 5 & 2 \\
\hline $\mathrm{III}$ & 13 & 4 \\
\hline
\end{tabular}




\subsection{Therapie}

\subsubsection{Operation}

Alle 64 Patienten der Studie wurden operiert. In Abhängigkeit von der LaurenKlassifikation und der Tumorlokalisation wurden drei unterschiedliche Formen der Gastrektomie durchgeführt: die totale, subtotale und die erweiterte Gastrektomie.

Bei über der Hälfte der Patienten ( $n=37 / 64)$ wurde eine totale Gastrektomie durchgeführt.

Insgesamt 8 Patienten erhielten eine subtotale Gastrektomie. Diese Operation konnte aufgrund des kleineren Sicherheitsabstandes bei der Histologie des intestinalen Typs nach Lauren, einer geringen Tumorgröße und der Lokalisation im Antrum durchgeführt werden.

Eine erweiterte Gastrektomie wurde insgesamt 19 mal durchgeführt. Die Erweiterung erfolgte aufgrund des magenüberschreitenden Tumorwachstums in Form der Splenektomie, der Pankreaskapselresektion oder der partiellen Oesophagusresektion.

Bei fast allen Patienten ( $n=61 / 64)$ wurde eine D2-Lymphadenektomie durchgeführt. 25/26 Patienten der Gruppe 1 und 36/38 Patienten aus Gruppe 2 wurden D2reseziert.

Eine D1-Resektion wurde bei insgesamt 3/64 Patienten aufgrund des frühen Tumorstadiums und des fehlenden Hinweises auf Lymphknotenmetastasen durchgeführt.

Tabelle 10 Operationsverfahren

\begin{tabular}{|l|c|c|}
\hline & Gruppe1 & Gruppe2 \\
\hline Totale Gastrektomie & 17 & 20 \\
Subtotale Gastrektomie & 1 & 7 \\
Erweiterte Gastrektomie & 8 & 11 \\
\hline D1 & 1 & 2 \\
D2 & 25 & 36 \\
\hline
\end{tabular}




\subsubsection{Resektionsstatus}

Resektionsstatus: Gruppe 1 und 2

Die als kurativ erachtete und für die Prognose entscheidende R0-Resektion konnte insgesamt 48mal erfolgen. 20/26 Patienten in Gruppe 1 und 28/38 aus Gruppe 2 wurden R0-reseziert.

Eine R1-Resektion erfolgte insgesamt 16mal.

\section{Resektionsstatus: Gruppe 1}

Eine vollständige Tumorentfernung wurde bei 17 Respondern und bei 3 NichtRespondern erzielt. Insgesamt 6 mal erfolgte eine R1-Resektion.

Tabelle 11 Resektionsstatus in Abhängigkeit vom Response

\begin{tabular}{|l|c|c|}
\hline & R0 & R1 \\
\hline Response $(\mathrm{n}=20)$ & 17 & 3 \\
\hline Nicht-Responder $(\mathrm{n}=6)$ & 3 & 3 \\
\hline
\end{tabular}

Resektionsstatus: Tumorstadium

\section{Gruppe 1}

6 der insgesamt 9 UICC-Stadien Ib/II und 12 der 17 UICC-Stadien III wurden vollständig reseziert. 6 mal erfolgte eine R1-Resektion.

\section{Gruppe 2}

Von den 23 UICC-Stadien Ib/II erfolgte 18mal eine R0-Resektion und von den 15 UICCStadien III 8mal. 


\subsubsection{Komplikationen}

35/64 Patienten hatten postoperativ Komplikationen.

Bei insgesamt 12 Patienten, häufiger in Gruppe 2, traten allgemeine Komplikationen wie Pneumonie, Lungenembolie, eine tiefe Beinvenenthrombose (TVT) oder einen Harnwegsinfekt auf.

Chirurgischen Komplikationen waren in beiden Gruppen ähnlich häufig. Anastomoseninsuffizienz, Wundheilungsstörungen ebenso verzögerter Kostaufbau infolge einer Schwellung im Bereich der Anastomose und lleuserscheinungen, einmal als Bridenileus und zweimal paralytisch infolge reflektorischer Darmlähmung, traten auf.

Tabelle 12 Postoperative Komplikationen in Gruppe 1 und 2

\begin{tabular}{|l|c|c|}
\hline $\begin{array}{l}\text { chirurgische } \\
\text { Komplikationen }\end{array}$ & Gruppe 1 $(\mathrm{n}=26)$ & Gruppe 2 $(\mathrm{n}=38)$ \\
\hline Anastomoseninsuffizienz & 3 & 4 \\
\hline Verzögerter Kostaufbau & 0 & 5 \\
\hline Ileus & 2 & 5 \\
\hline Wundheilungsstörung & 3 & \\
\hline & & \\
allgemeine & & 6 \\
Komplikationen & & 2 \\
\hline Pneumonie & 1 & 1 \\
\hline Lungenembolie & 0 & 1 \\
\hline TVT & 1 & \\
\hline Harnwegsinfekt & 0 & \\
\hline
\end{tabular}




\subsection{Chemotherapie}

26 Patienten der Studie (Gruppe 1) wurden gemäß PELF-Protokoll neoadjuvant chemotherapiert. Nach dem klassischen Schema des PELF-Protokolls, wie es in der vorliegenden Studie durchgeführt wurde, werden den Patienten in 2 Zyklen à 6 Wochen die Chemotherapeutika Cisplatin, Epirubicin, Leucovorin und 5 - Fluoruracil verabreicht.

Tabelle 13 Chemotherapieschema PELF-Protokoll Indikation Magenkarzinom

\begin{tabular}{|c|c|l|l|}
\hline \multicolumn{6}{|l|}{ PELF (modifiziert ${ }^{\star}$ ) } \\
\hline \multicolumn{2}{|l|}{ Chemotherapie } \\
\hline Woche & Tag & Substanz & Dosierung \\
\hline $1-6$ & 1 & Cisplatin/Platiblastin & $40 \mathrm{mg} / \mathrm{m}^{2}$ \\
\hline $1-6$ & 1 & Epirubicin/Farmorubicin & $35 \mathrm{mg} / \mathrm{m}^{2}$ \\
\hline $1-6$ & 1 & Calciumfolinat/Leukovorin & $500 \mathrm{mg} / \mathrm{m}^{2}$ \\
\hline $1-6$ & 1 & 5-Fluoruracil/5-FU Lederle & $500 \mathrm{mg} / \mathrm{m}^{2}$ \\
\hline
\end{tabular}

${ }^{*}$ Im Originalprotokoll mit Gluthation

1Zyklus $=6$ Wochen Behandlung, Wiederholung nach Woche 8

\subsection{Vollständigkeit}

Insgesamt 18/26 Patienten erhielten die Chemotherapie vollständig und 8mal wurde nach dem ersten Zyklus abgebrochen. Bei Respondern und Nicht-Respondern war das Verhältnis von vollständiger Therapie zu frühzeitigem Abbruch ähnlich.

Gründe für den frühzeitigen Abbruch waren bei den Respondern In einem Fall eine vollständige Tumorremission und Zeichen der Toxizität wie Neutropenie $(n=2 / 20)$ und starke allgemeine Unverträglichkeit $(n=3 / 20)$. 
Bei den Nicht-Respondern wurde die Therapie wegen Tumorprogress $(n=2 / 6)$ und wegen zusätzlicher starker allgemeiner Unverträglichkeit $(n=1 / 6)$ nach dem ersten Zyklus abgebrochen.

Tabelle 14 Anzahl der Zyklen der Chemotherapie $(n=26)$

\begin{tabular}{|l|c|c|}
\hline Vollständigkeit & Responder & Nicht-Responder \\
\hline 2 Zyklen & 14 & 4 \\
\hline 1 Zyklus & 6 & 2 \\
& $-1^{*}$ volle Remission & $-2^{*}$ Tumorprogress \\
\hline
\end{tabular}

\subsubsection{Response}

Im Anschluss an den jeweils letzten Zyklus erfolgte die Ermittlung des Response mittels Endosonographie und CT.

Insgesamt 5mal kam ein kompletter und 15mal ein partieller Response vor. Dabei zeigte sich ein vollständiger Regress besonders häufig im Stadium III.

Bei allen UICC-Stadien (Ib-III) zusammen war ein partieller Response häufiger $(n=15 / 26)$ als ein vollständiger Response $(n=5 / 26)$.

Tabelle 15 Response der Tumorstadien in bildgebender Diagnostik $(n=26)$

\begin{tabular}{|l|c|c|}
\hline & $\begin{array}{c}\text { UICC lb/ II } \\
(\mathrm{n}=9 / 26)\end{array}$ & $\begin{array}{c}\text { UICC III } \\
(\mathrm{n}=17 / 26)\end{array}$ \\
\hline $\begin{array}{c}\text { Response }(\mathrm{n}=20) \\
- \text { CR }\end{array}$ & -1 & -4 \\
- PR & -6 & -9 \\
\hline Kein Response & -2 & -4 \\
\hline
\end{tabular}




\subsubsection{Regressionsgrade nach JRSGC (1995)}

Postoperativ wurden die Regressionsgrade histologisch ermittelt und dabei Unterschiede zwischen den Ergebnissen der präoperativen bildgebenden Diagnostik und den postoperativ histologisch ermittelten festgestellt.

Bei 3 Patienten war ein Regressionsgrad 3 festzustellen; das Gewebe war komplett nekrotisch. In 9 Fällen wurden minimales, aber noch vitales Tumorgewebe und submuköse subtotale Vernarbungen beobachtet, dies entsprach einem Regressionsgrad 2.

Pathohistologisch können drei verschiedene Regressionsgrade (JRSGC (1995)) unterschieden werden: 1-3 (0=keine Regression).

\section{Tabelle 16 Einteilung der Regressionsgrade}

- Grad 1 (geringe Regression)

Nekrose oder Verschwinden des Tumors und / oder zelluläre oder strukturelle Veränderungen in weniger als $1 / 3$ des Tumors ( Grad 1a) oder mehr als $1 / 3$ aber nicht in 2/3 (Grad 1b)

- Grad 2 (mäßig-gradige Regression)

Nekrose oder Verschwinden des Tumors in mehr als 2/3 des Tumors, aber noch vitale Tumorzellen erkennbar

- Grad 3 (ausgeprägte Regression)

Tumor komplett nekrostisch und / oder durch Fibrose ersetzt, keine vitalen Tumorzellen nachweisbar 


\subsubsection{Tumorstadium, Restaging (UICC)}

Nach Chemotherapie erfolgte das Restaging mittels Endosonographie und CT.

Die Gegenüberstellung der zu den unterschiedlichen Zeitpunkten ermittelten Stadien zeigte: nach Chemotherapie hatte Stadium III an Häufigkeit abgenommen und die Stadien Ib und II waren häufiger diagnostiziert worden.

Tabelle 17 Tumorstadien vor und nach Chemotherapie (UICC)

\begin{tabular}{|l|c|c|}
\hline UICC & vor Chemotherapie & nach Chemotherapie \\
\hline Ib & 2 & 6 \\
\hline II & 7 & 9 \\
\hline III & 17 & 9 \\
\hline IV & 0 & 2 \\
\hline
\end{tabular}

Postoperativ wurden im Vergleich zum Restaging das Stadium III seltener ( $n=3 / 26)$ und die frühen Stadien Ib $(n=10 / 26)$ und II $(n=7 / 26)$ häufiger diagnostiziert.

Tabelle 18 Stadienverteilung nach Chemotherapie und nach Operation (UICC)

\begin{tabular}{|l|c|c|}
\hline UICC & vor Chemotherapie & $\begin{array}{c}\text { nach Operation } \\
\text { (histopathologisch) }\end{array}$ \\
\hline I & 2 & $1 \mathrm{lb}$ \\
& & $1 \mathrm{III}$ \\
\hline II & 7 & $1 \mathrm{lb}$ \\
& & $4 \mathrm{II}$ \\
& 17 & $2 \mathrm{IV}$ \\
\hline IV & & $8 \mathrm{Ib}$ \\
& & $3 \mathrm{II}$ \\
& & $1 \mathrm{III}$ \\
\hline
\end{tabular}




\subsubsection{Tumorstadium, postoperativ (UICC)}

Die prätherapeutisch im Rahmen des Stagings diagnostizierten Tumorstadien wurden mit den postoperativ histopathologisch eruierten verglichen.

In Gruppe 1 lag nach Chemotherapie (CTx) 18mal statt wie prätherapeutisch 9mal ein früheres Stadium (Ib/II) vor und insbesondere Stadium III hatte an Häufigkeit abgenommen. Die Zunahme früherer und die Abnahme fortgeschrittener Stadien entspricht einem Downstaging.

Tabelle 19 Verteilung der Tumorstadien vor CTx vs. nach Operation In Gruppe 1

\begin{tabular}{|l|c|c|}
\hline UICC & vor Chemotherapie & $\begin{array}{c}\text { nach Operation } \\
\text { (histopathologisch) }\end{array}$ \\
\hline Ib & 2 & 10 \\
\hline II & 7 & 7 \\
\hline III & 17 & 3 \\
\hline IV & 0 & 6 \\
\hline
\end{tabular}

In Gruppe 2 musste in 18/38 Fällen das prätherapeutisch mittels CT und EUS ermittelte Tumorstadium postoperativ korrigiert werden. Insbesondere die Stadien Ib, II und III waren unsicher eingeschätzt worden.

Tabelle 20 Vergleich der Tumorstadien vor und nach Operation in Gruppe 2 ( $n=38)$

\begin{tabular}{|l|c|c|}
\hline UICC & Zu Beginn der Therapie & histopathologisch \\
\hline Ib & 10 & 16 \\
\hline II & 13 & 9 \\
\hline III & 15 & 7 \\
\hline IV & 0 & 6 \\
\hline
\end{tabular}




\subsection{Histologie}

\subsubsection{Klassifikation nach Lauren}

Die Lauren-Klassifikation wurde histologische anhand des intraoperativ entnommenen Tumorgewebes ermittelt.

Der intestinale Typ nach Lauren fand sich insgesamt am häufigsten ( $n=36 / 64)$. Der diffuse Typ kam insgesamt 22mal vor. Der Mischtyp wurde entsprechend der am niedrigsten differenzierten Zelle zum diffusen Karzinom gerechnet. Die Verteilung der Häufigkeiten war in beiden Gruppen ähnlich.

Tabelle 21 Lauren-Klassifikation

\begin{tabular}{|c|c|c|}
\hline & Gruppe1 $\quad n=26$ & Gruppe $2 \quad n=38$ \\
\hline Intestinal & 15 & 21 \\
\hline Diffus & 7 & 15 \\
\hline Mischtyp & 4 & 2 \\
\hline
\end{tabular}

\subsubsection{Klassifikation nach der WHO}

Die Klassifizierung nach der WHO geschieht im Rahmen der histopathologischen Aufarbeitung des intraoperativ entnommenen Tumorgewebes.

Das Adenokarzinom war am häufigsten $(n=51 / 64)$. Ein Siegelringszellkarzinom kam mit $13 \mathrm{mal}$ etwas seltener vor. Undifferenzierte Karzinome wurden nicht beobachtet. Die Häufigkeiten der Histologien waren in beiden Studiengruppen ähnlich.

Tabelle 22 Histologische Klassifizierung nach der WHO

\begin{tabular}{|c|c|c|}
\hline WHO-Klassifikation & Gruppe1 & Gruppe2 \\
\hline Adenokarzinom & 20 & 31 \\
\hline - papillär & 3 & 3 \\
\hline tubulär & 6 & 9 \\
\hline muzinös & 1 & 6 \\
\hline Siegelringzellkarzinom & 6 & 7 \\
\hline
\end{tabular}




\subsubsection{Grading}

Der Differenzierungsgrad wurde postoperativ histopathologisch ermittelt.

Insgesamt waren G3-Tumoren mit einer geringeren Differenzierung ( $n=37 / 64)$ häufiger als G1-2 Tumoren mit guter bis mäßiger Differenzierung.

In Gruppe 1 überwogen G3-Tumoren; 6mal kam ein G1-2 Tumor vor.

In Gruppe 2 kamen G3-Tumoren 17mal und G1-2 Tumoren 20mal vor.

Tabelle 23 Differenzierungsgrad

\begin{tabular}{|l|c|c|}
\hline Grading & Gruppe1 & Gruppe2 \\
\hline G1-2 & 6 & 21 \\
G3 & 20 & 17 \\
\hline
\end{tabular}




\section{Spätergebnisse}

\subsection{Rezidive}

Insgesamt 33/64 Patienten bekamen ein Rezidiv. Die Rezidivrate lag bei 51,6\%.

Ein Rezidiv war in Gruppe 1 ( $n=10 / 26)$ weniger häufig wie in Gruppe $2(n=23 / 38)$.

Tabelle 24 Häufigkeit der Rezidive

\begin{tabular}{|l|c|c|}
\hline & Gruppe 1 & Gruppe 2 \\
\hline Rezidiv & 10 & 23 \\
\hline kein Rezidiv & 16 & 15 \\
\hline
\end{tabular}

\subsubsection{Lokalisation}

Die häufigste Rezidivlokalisation war das lokoregionäre Rezidiv ( $n=13 / 64$ ).

3mal kam es in Gruppe $1(n=26)$ und 10mal in Gruppe 2 ( $n=38)$ vor; nach Chemotherapie war es weniger häufig wie nach alleiniger Operation.

Eine Peritonealkarzinose war insgesamt bei 7/64 Patienten zu beobachten und Fernmetastasen mit unterschiedlicher Lokalisation traten insgesamt 9mal auf. Die Häufigkeiten waren in beiden Gruppen ähnlich.

Lymphknotenmetastasen kamen bei 5 Patienten vor und wurden nur in Gruppe 2 diagnostiziert.

Bei insgesamt sieben Patienten ( $\mathrm{n}_{1}=2, \mathrm{n} 2=5$ ) wurden multiple Rezidivlokalisationen diagnostiziert. Es traten Leber-, Lungenmetastasen+/-Peritonealkarzinose plus Lymphknotenmetastasen auf. 
Tabelle 25 Rezidiv-Lokalisation

\begin{tabular}{|c|c|c|}
\hline Rezidiv-Lokalisation & $\begin{array}{c}\text { Gruppe1 } \\
(n=26)\end{array}$ & $\begin{array}{l}\text { Gruppe2 } \\
(n=38)\end{array}$ \\
\hline Lokalrezidiv & 3 & 10 \\
\hline - Anastomose. & -3 & -9 \\
\hline Peritonealkarzinose & 3 & 4 \\
\hline Lymphknotenmetastasen & 0 & 5 \\
\hline - paraaortal & & -2 \\
\hline - retroperitoneal & & 2 \\
\hline - kl.Kurvatur & & 1 \\
\hline Fernmetastasen & 4 & 5 \\
\hline Nebenniere & 1 & 0 \\
\hline Sigma & 1 & 0 \\
\hline Leber & 1 & 4 \\
\hline Dünndarm & 1 & 0 \\
\hline Lunge & 0 & 1 \\
\hline
\end{tabular}




\section{2 Überlebensraten (Gesamtkollektiv)}

Zunächst wurde das Überleben des Gesamtkollektivs ( $n=64)$ analysiert und Subgruppenanalysen nach verschiedenen Kriterien durchgeführt.

\subsubsection{Tumorstadium (UICC), prätherapeutisch}

UICC-Stadium Ib ( $n=12 / 64)$ hatte eine 5-JÜR von 58,4\%.

Im Stadium II ( $n=20 / 64)$ lag die 5-JÜR bei 39,3\%. Das mediane Überleben lag bei 3,6 Jahren bei einer Streubreite von 1,2 bis 6,0 Jahren.

UICC-Stadium III ( $n=28 / 64$ )hatte eine 5-JÜR von 41,5\%. Das mediane Überleben lag bei 2,8 Jahren bei einer Streubreite von 1,9 bis 3,7 Jahren.

Mit Stadium IV $(n=4 / 64)$ hat keiner der Patienten 5 Jahre überlebt. Das mediane Überleben lag bei 0,4 Jahren bei einer Streubreite von 0,0 und 1,6 Jahren.

Der Unterschied in der Überlebensprognose erwies sich statistisch als signifikant $(p=0,014)$.

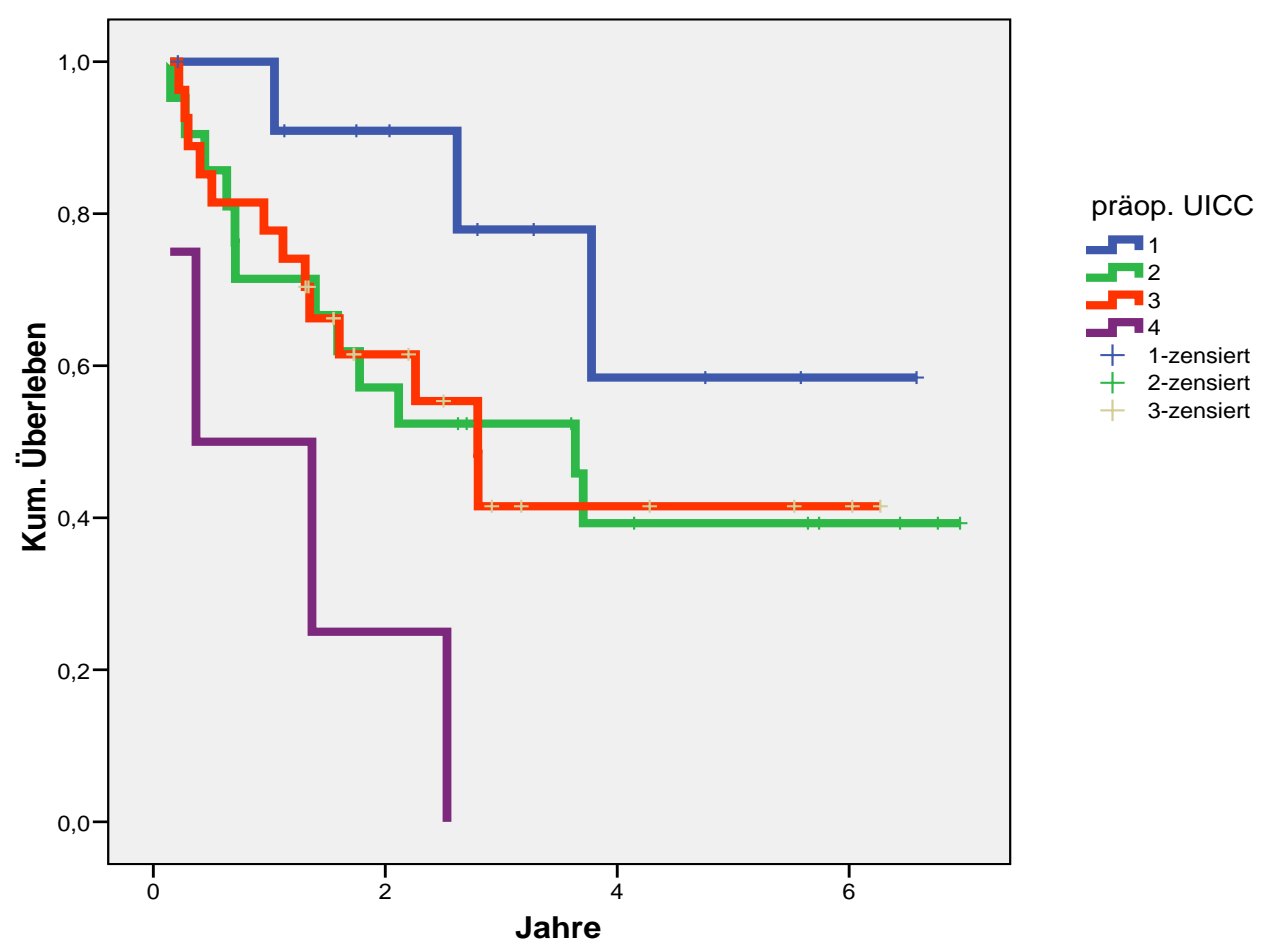

Abb. 1 Überlebensraten der Tumorstadien UICC, prätherapeutisch 


\subsubsection{Tumorstadium (UICC), postoperativ}

UICC Stadium Ib ( $n=26 / 64)$ hatte eine 5-JÜR von 66,5\%.

Im Stadium II ( $n=17 / 64)$ lag die 5-JÜR bei 45,5\%. Das mediane Überleben lag bei 2,8 Jahren.

UICC-Stadium III ( $n=10 / 64)$ hatte eine 5-JÜR von 15,9\%. Das mediane Überleben lag bei 2,5 Jahren bei einer Streubreite von 0,4 bis 4,5 Jahren.

Mit Stadium IV ( $n=11 / 64)$ lag die 5-JÜR bei 20,5\%. Das mediane Überleben lag bei 0,9 Jahren bei einer Streubreite von 0,2 bis 1,7 Jahren.

Der Unterschied in der Überlebensprognose erwies sich statistisch als signifikant $(p=0,001)$.

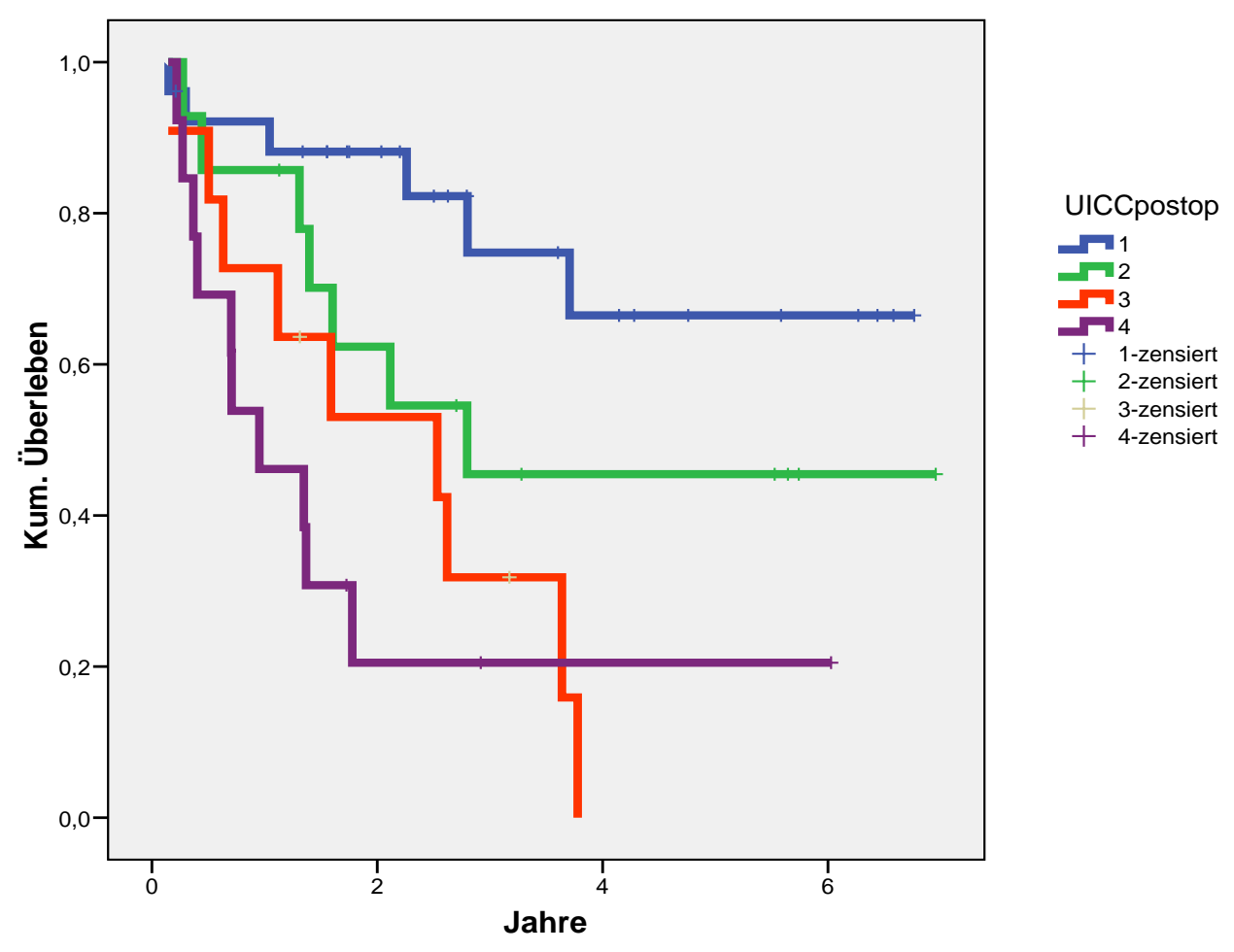

Abb. 2 Überlebensraten der Tumorstadien UICC, postoperativ 


\subsubsection{Tumorstadium (UICC I/II und III/IV), prätherapeutisch}

UICC-Stadium Ib und II ( $n=32 / 64)$ zusammen hatten eine 5-JÜR von 45,8\%. Das mediane Überleben lag bei 3,7 Jahren.

Stadium III und IV ( $n=32 / 64$ ) hatten eine 5-JÜR von 34,3\%. Das mediane Überleben lag bei 2,5 Jahren bei einer Streubreite von 1,1 bis 4,0 Jahren.

Der Unterschied in der Überlebensprognose erwies sich statistisch als nicht signifikant $(p=0,192)$.

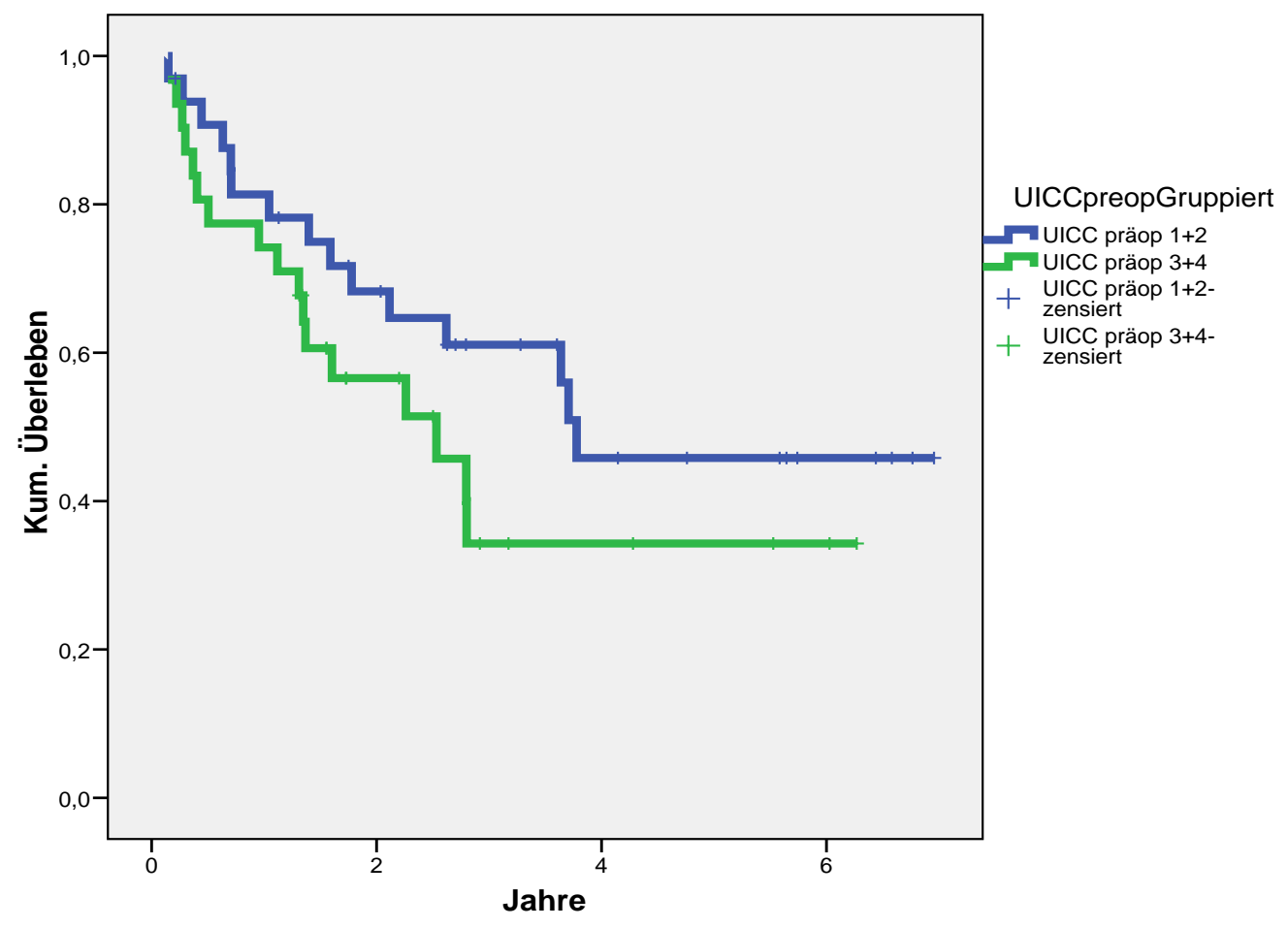

Abb. 3 Überlebensraten der Tumorstadien UICC Ib/II und III/IV (prätherapeutisch) 


\subsubsection{Lymphknotenbeteiligung}

Patienten ohne Lymphknotenmetastasen ( $n=26 / 64)$ hatten eine 5-JÜR von 66,1\%.

Patienten mit Lymphknotenmetastasen ( $n=38 / 64$ ) hatten eine 5-JÜR von $24,7 \%$. Das mediane Überleben lag bei 2,1 Jahren und die Streubreite zwischen 0,9 und 3,4 Jahren.

Der Unterschied in der Überlebensprognose erwies sich als statistisch signifikant $(p=0,01)$.

Überlebensfunktionen

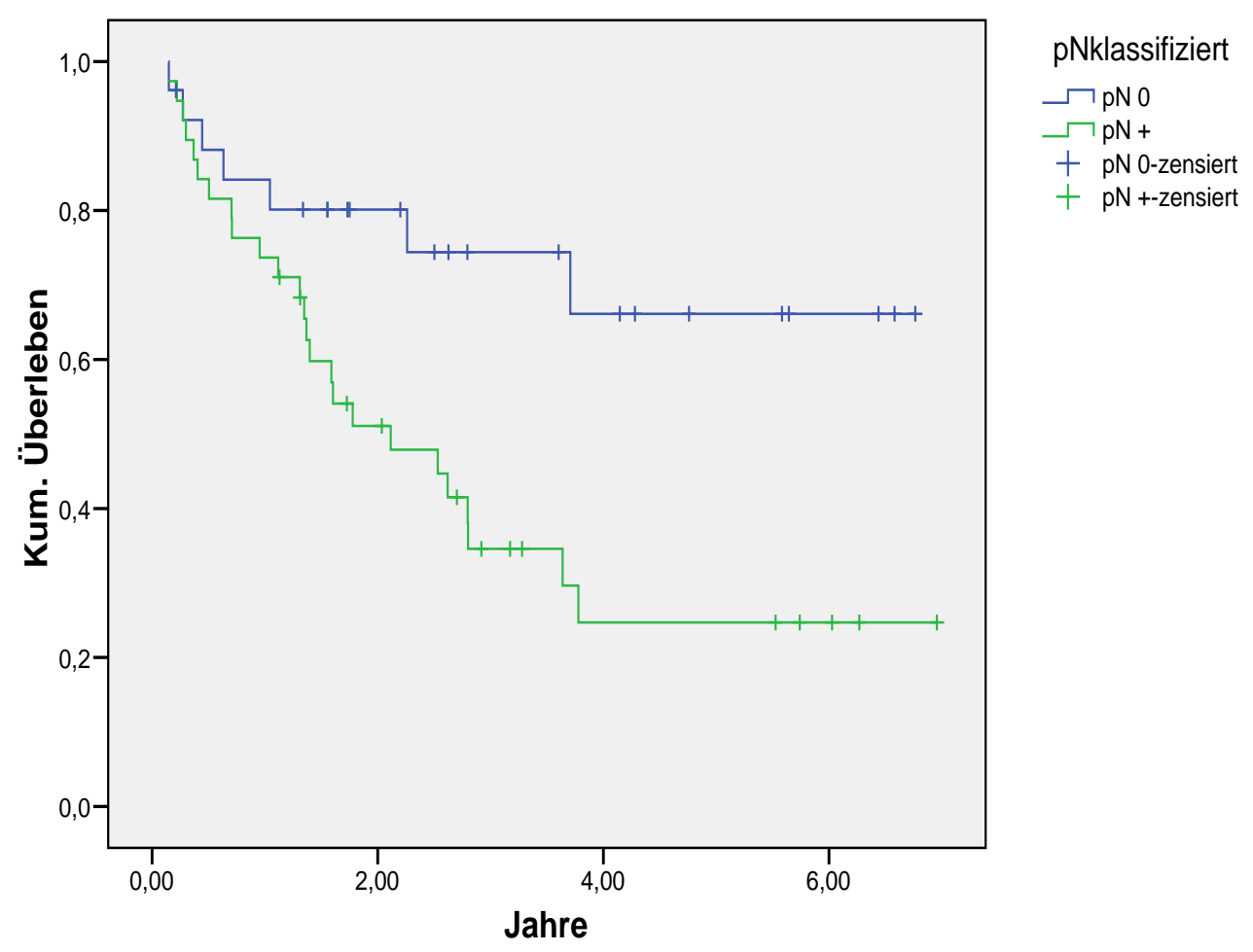

Abb. 4 Überlebensraten mit/ohne Lymphknotenmetastasen 


\subsubsection{Tumorgrading}

Patienten G1-2-Tumoren ( $n=27 / 64)$ hatten eine 5-JÜR von 46,1\%. Das mediane Überleben lag bei 3,5 Jahren.

Patienten mit G3-Tumoren ( $n=37 / 64)$ hatten eine 5-JÜR von 36,3\%. Das mediane Überleben lag bei 2,5 Jahren, die Streubreite zwischen 1,0 und 4,1 Jahren.

Der Unterschied in der Überlebensprognose erwies sich statistisch als nicht signifikant $(p=0,173)$.

Überlebensfunktionen

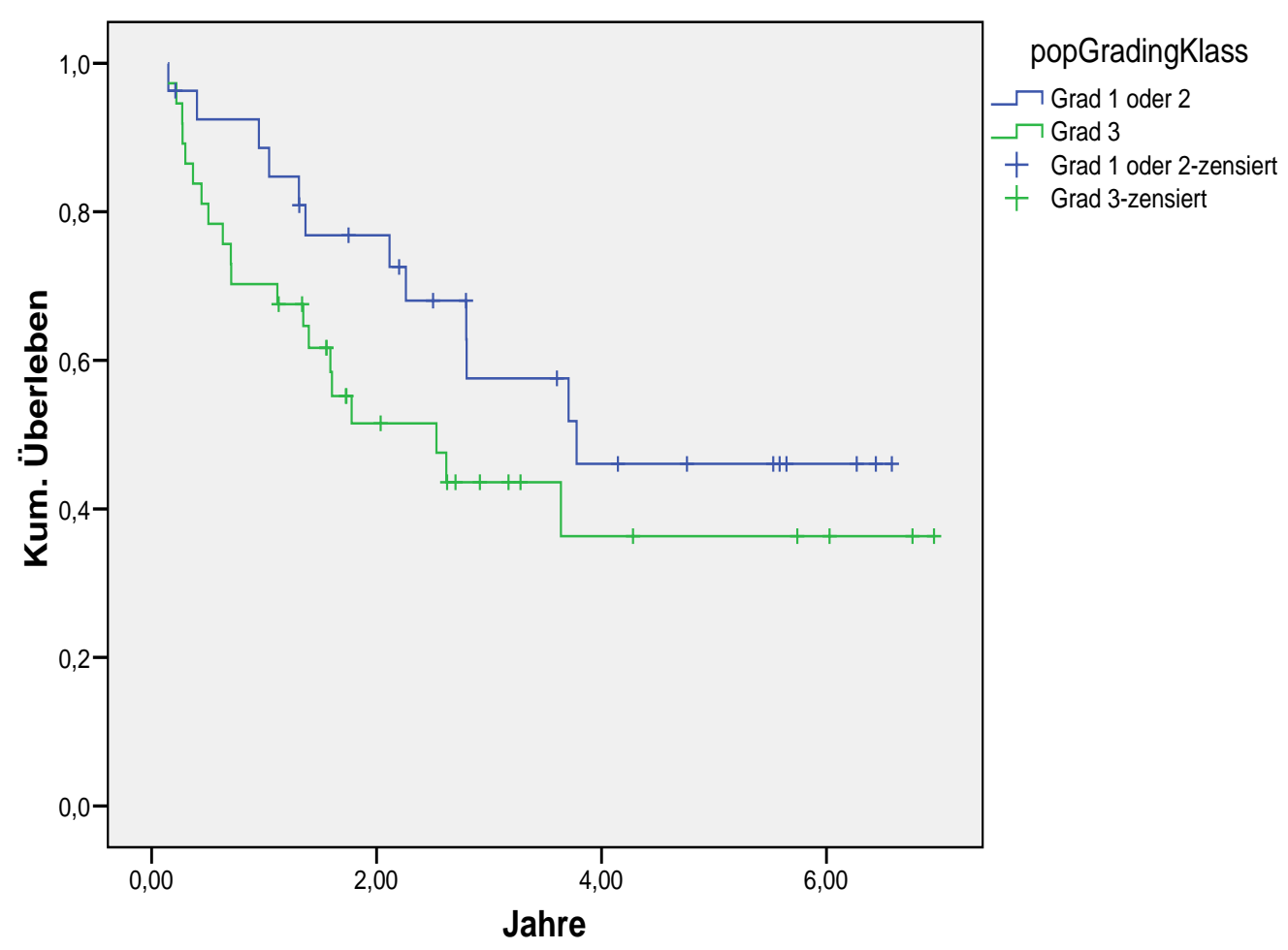

Abb. 5 Überlebensraten bei gutem (G1-2) und mäßigem (G3) Differenzierungsgrad 


\subsubsection{Resektionsstatus}

Nach R0-Resektion ( $n=48 / 64$ ) lag die 5-JÜR bei 51,5\%.

Von Patienten mit palliativer Resektion $(n=16 / 64)$ hat keiner 5 Jahre überlebt. Das mediane Überleben lag bei 0,63 Jahren, die Streubreite zwischen 0,24 und 1,0 Jahren.

Der Unterschied in der Überlebensprognose erwies sich als statistisch signifikant $(p=0,000)$.

In der Cox-Regression zeigte sich, dass der Resektionsstatus R+ oder R0 ein unabhängiger Prognoseparameter ist.

\section{Überlebensfunktionen}

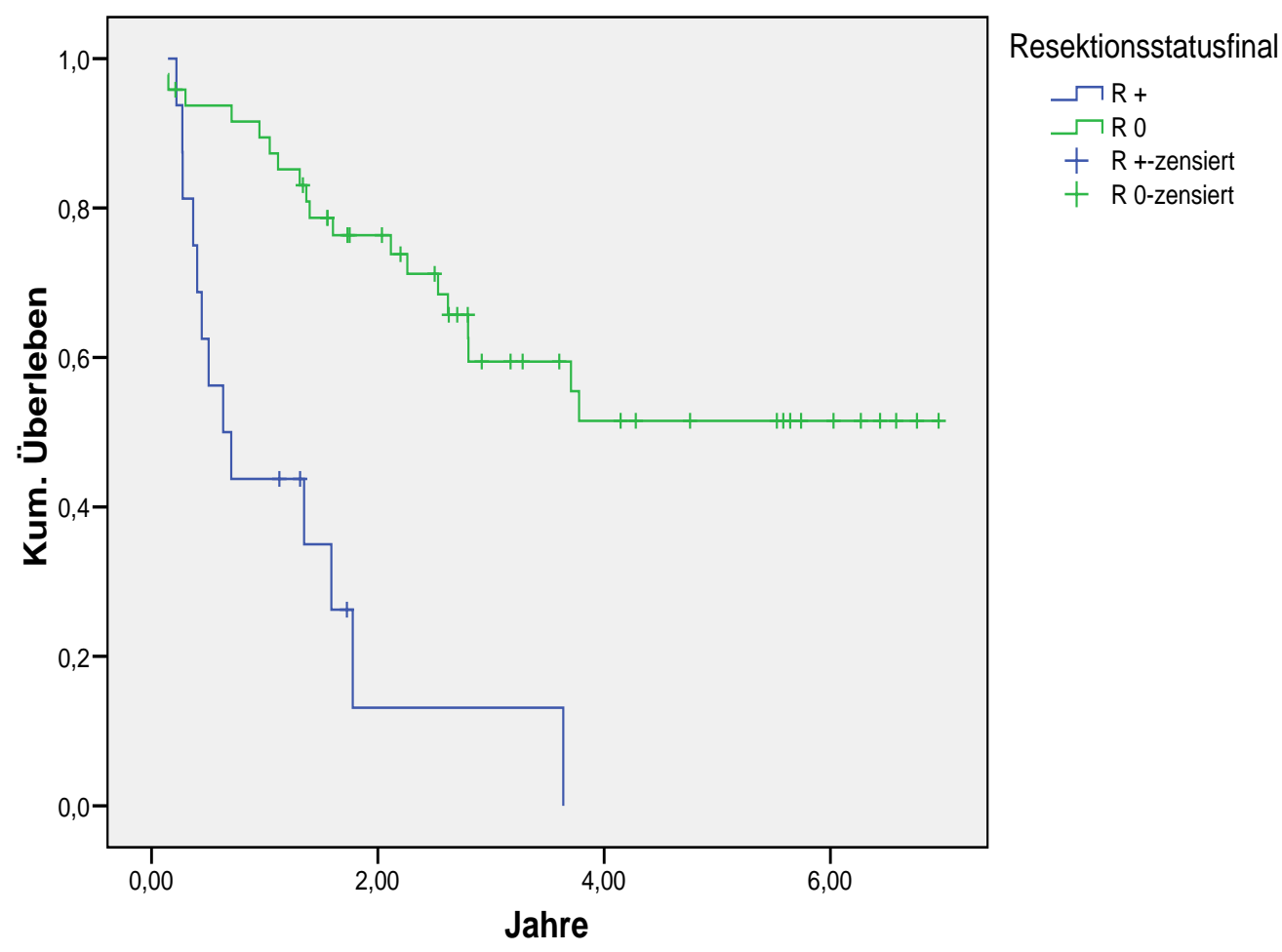

Abb. 6 Überlebensraten bei R0- und R+-Resektion 


\subsubsection{Fernmetastasen}

Mit Fernmetastasen $(n=7 / 64)$ hat kein Patient 5 Jahre überlebt. Das mediane Überleben lag bei 0,4 Jahren, die Streubreie zwischen 0,31 und 0,49 Jahren.

Ohne Fernmetastasen ( $n=57 / 64$ ) lag die 5 -JÜR bei $45 \%$, das mediane Überleben bei 3,7 Jahren und die Streubreite zwischen 2,25 und 5,2 Jahren.

Der Unterschied in der Überlebensprognose erwies sich als statistisch signifikant $(p=0,000)$.

Überlebensfunktionen

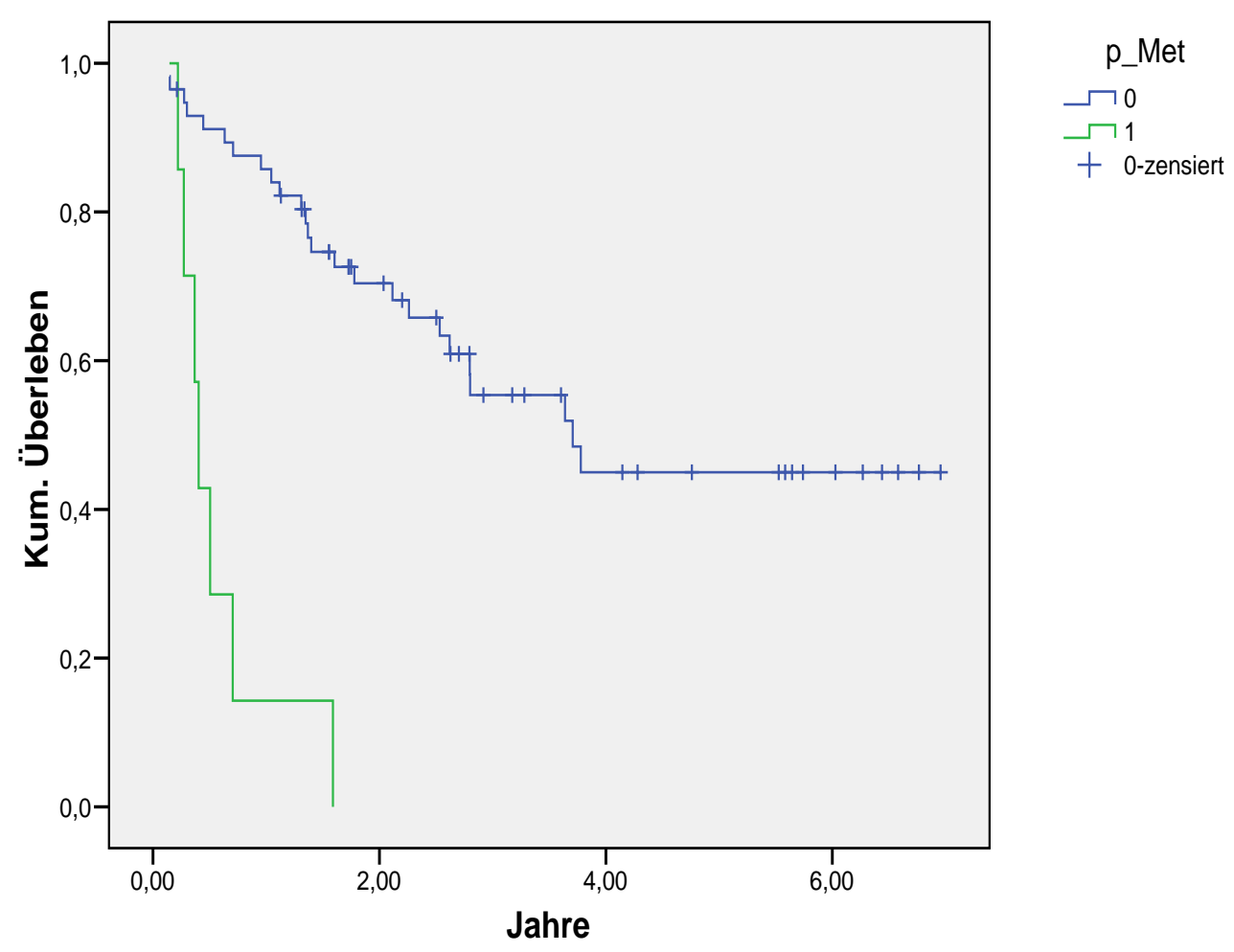

Abb. 7 Überlebensraten mit/ohne Fernmetastasen 


\subsubsection{R0-/R+-Resektion und Fernmetastasen}

R0-resezierte Patienten ohne Fernmetastasen überlebten 51,5\%.

Palliativ resezierte Patienten ohne Fernmetastasen überlebten keine 5 Jahre. Das mediane Überleben lag bei 1,4 Jahren, die Streubreite zwischen 0,34 und 2,4 Jahren.

Patienten mit Fernmetastasen überlebten keine 5 Jahre. Das mediane Überleben lag bei 0,4 Jahren, die Streubreite zwischen 0,31 und 0,29 Jahren.

Der Unterschied in der Überlebensprognose erwies sich als statistisch signifikant $(p=0,000)$.

\section{Überlebensfunktionen}

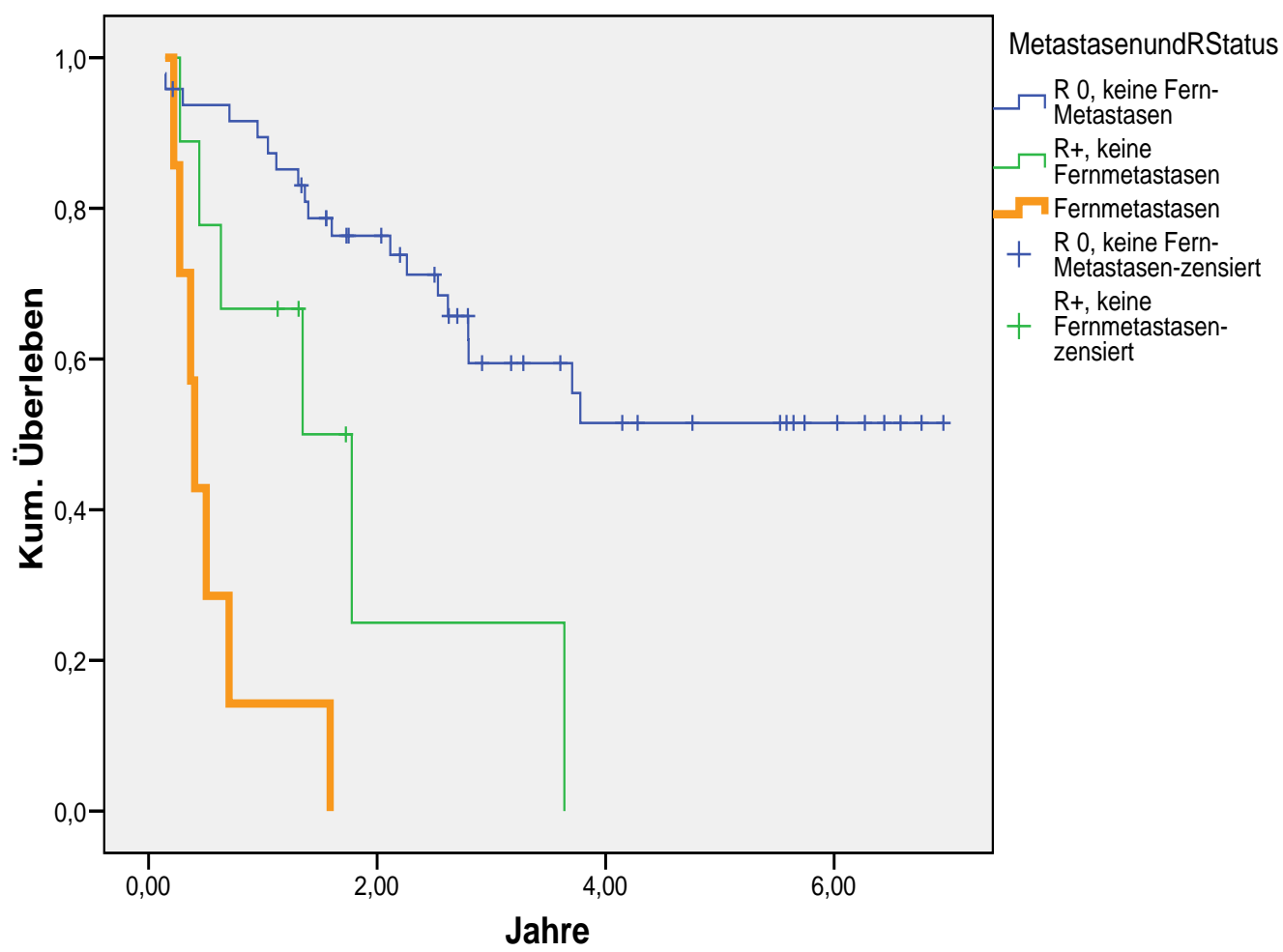

Abb. 8 Überlebensraten bei R0-/R+-Resektion und Fernmetastasen 


\subsubsection{Chemotherapie vs. nur Operation}

Nach Chemotherapie $(n=26 / 64)$ lag das Überleben bei $37,5 \%$. Das mediane Überleben betrug 2,6 Jahre bei einer Streubreite von 1,2 bis 3,3 Jahren.

Nur operierte Patienten ( $n=38 / 64)$ hatten eine 5-JÜR von $42,3 \%$. Das mediane Überleben lag bei 3,7 Jahren und die Streubreite zwischen 2,4 und 4,9 Jahren.

Die Unterschiede im Überleben waren statistisch nicht signifikant $(p=0,580)$.

Überlebensfunktionen

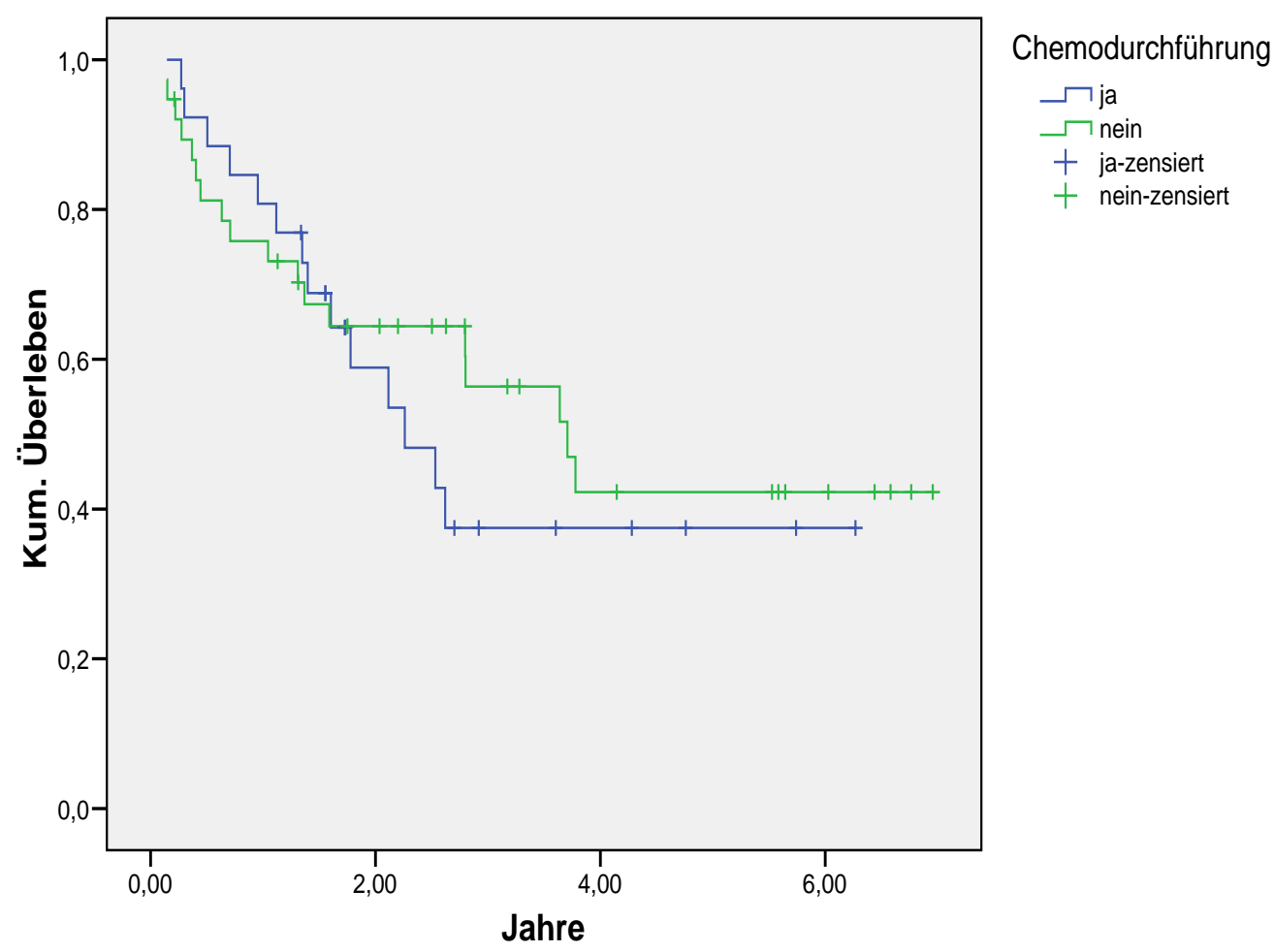

Abb. 9 Überlebensraten nach Chemotherapie versus nach nur Operation 
Nun wurden die Überlebensraten der Responder $(n=20 / 64)$, der NichtResponder ( $n=6 / 64)$ und der nur Operierten $(n=38 / 64)$ analysiert.

\subsubsection{Responder versus Nicht-Responder versus nur Operierte}

Responder hatten eine 5-JÜR von 44,6\%. Die mediane Überlebenszeit betrug 2,6 Jahre bei einer Streubreite von 2,0 bis 3,2 Jahren.

Nicht-Responder hatten eine 5 -JÜR von $20 \%$, es hat ein Patient 5 Jahre überlebt. Das mediane Überleben lag bei 1,1 Jahren bei einer Streubreite von 0,0 bis 2,4 Jahren.

Nur Operierte hatten eine 5-JÜR von 42,3\%. Das mediane Überleben lag bei 3,7 Jahren und die Streubreite zwischen 2,4 und 4,9 Jahren.

Die Unterschiede im Überleben erwiesen sich als nicht signifikant $(p=0,283)$.

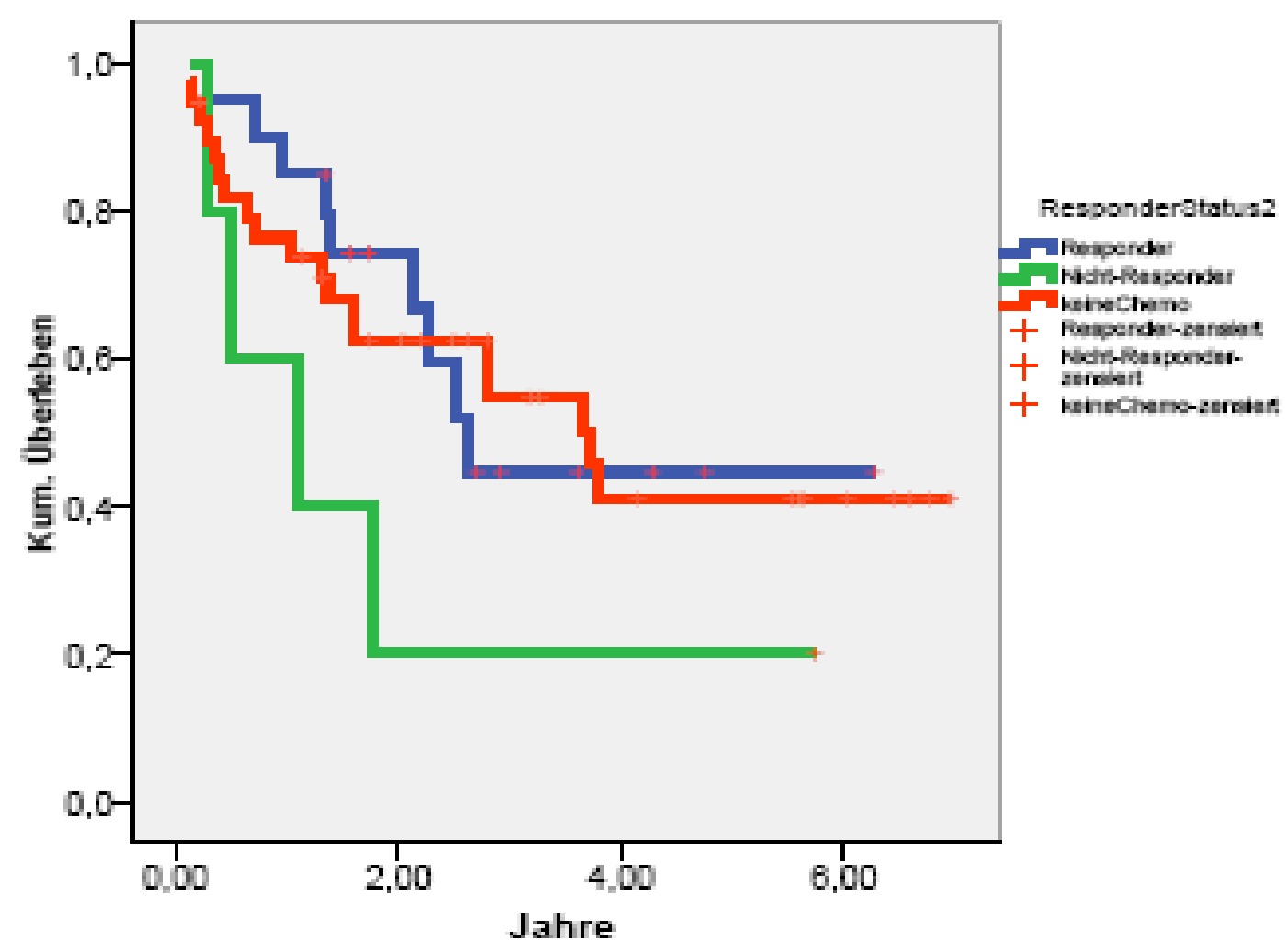

Abb. 10 Überlebensraten von Respondern, Nicht-Respondern und nur Operierten 


\subsubsection{Tumorstadium (UICC), prätherapeutisch}

Responder mit UICC-Stadien Ib/II ( $n=7 / 20$ ) hatten eine 5-JÜR von 42,9\%. Das mediane Überleben lag bei 2,6 Jahren bei einer Streubreite von 1,3 und 3,9 Jahren.

Nicht-Responder ( $n=2 / 6)$ hatten eine 5 -JÜR von $50 \%$ bei einem medianen Überleben von 1,8 Jahren.

Nur operierte Patienten mit frühem Tumorstadium ( $n=23 / 38$ ) hatten eine 5-JÜR von $48,6 \%$ bei einem medianen Überleben von 3,8 Jahren.

Der Unterschied in der Überlebensprognose erwies sich statistisch als nicht signifikant $(p=0,838)$.

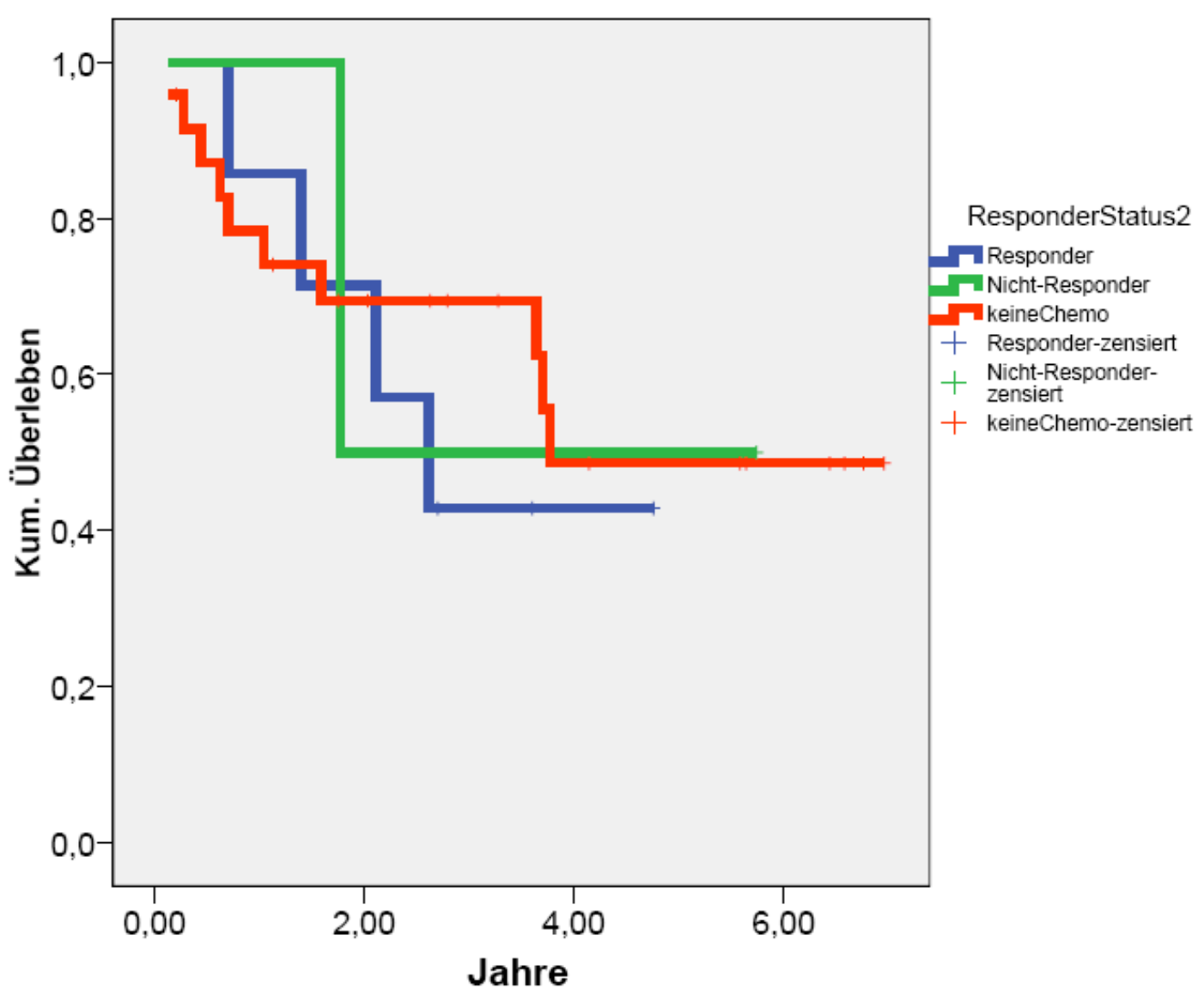

Abb. 11 Überlebensraten bei frühem Tumorstadium, UICC Ib/II, prätherapeutisch 
Responder im Stadium III/IV ( $n=13 / 20$ ) hatten eine 5-JÜR bei 45,7\%. Das mediane Überleben lag bei 2,5 Jahren.

Von den Nicht-Respondern ( $n=4 / 6)$ überlebte kein Patient mit spätem Tumorstadium 5 Jahre. Das mediane Überleben lag bei 0,5 Jahren bei einer Streubreite von 0,1 bis 0,9 Jahren.

Nur operierte Patienten mit fortgeschrittenem Tumorstadium ( $n=15 / 38)$ hatten eine 5JÜR von 31,1\%. Das mediane Überleben lag bei 2,8 Jahren bei einer Streubreite von 0,5 bis 5,0 Jahren.

Der Unterschied in der Überlebensprognose erwies sich als statistisch signifikant $(p=0,010)$.

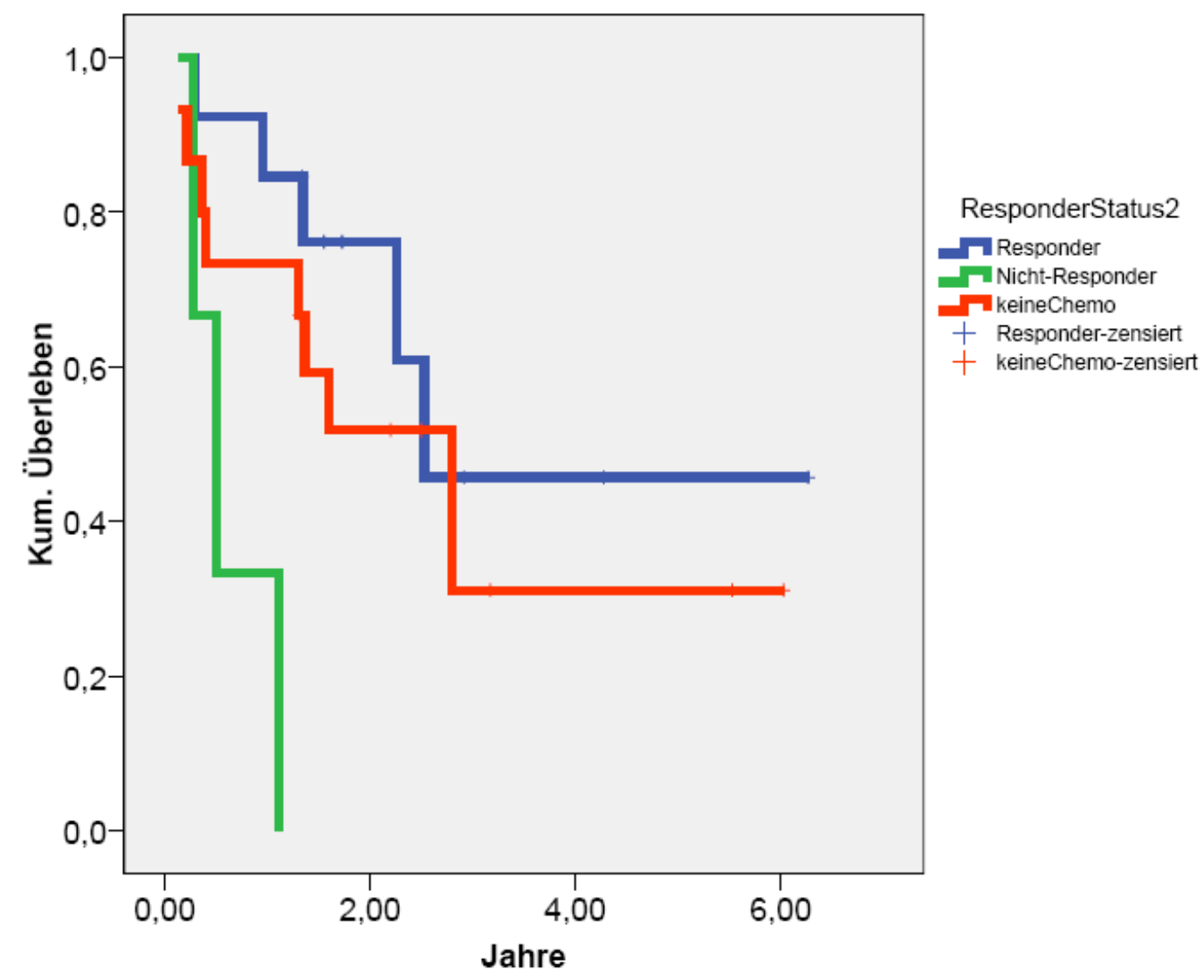

Abb. 12 Überlebensraten bei spätem Tumorstadium, UICC III/IV. prätherapeutisch 


\subsubsection{Tumorstadium (UICC), postoperativ}

Responder mit UICC Stadien Ib/II ( $n=13 / 20)$ hatten eine 5-JÜR von 59,9\%. (Nicht-Responder konnten aufgrund ihrer geringen Anzahl $(n=1 / 6)$ nicht errechnet werden.)

Bei den nur Operierten ( $n=26 / 38$ ) lag die 5-JÜR mit Stadium I/II bei 56,1\%.

Der Unterschied in der Überlebensprognose erwies sich statistisch als nicht signifikant $(p=0,759)$.

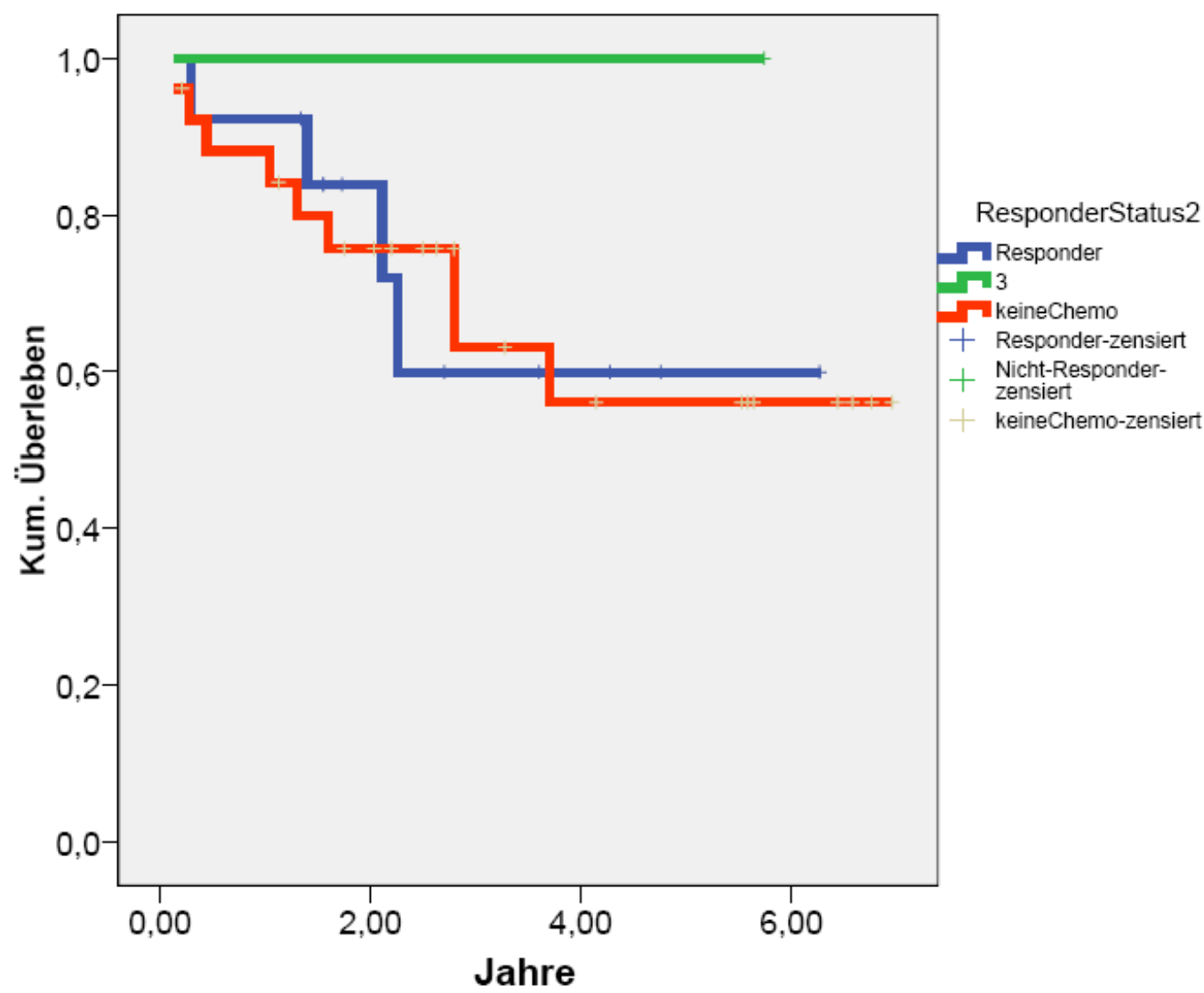

Abb. 13 Überlebensraten bei frühem Tumorstadium (UICC Ib/II), postoperativ 
Responder mit UICC III/IV ( $n=7 / 20)$ hatten eine 5-JÜR von 19\%. Das mediane Überleben lag bei 2,5 Jahren bei einer Streubreite von 0,1 bis 4,9 Jahren.

Von den Nicht-Respondern $(n=4 / 6)$ überlebte kein Patient mit fortgeschrittenen Tumorstadium 5 Jahre. Das mediane Überleben lag bei 0,5 Jahren bei einer Streubreite von 0,0 bis 1,3 Jahren.

Nur Operierte mit Stadium III/IV ( $n=13 / 38)$ hatten eine 5-JÜR von 12\%. Das mediane Überleben lag bei 1,30 Jahren bei einer Streubreite von 0,1 bis 2,6 Jahren.

Der Unterschied in der Überlebensprognose erwies sich als statistisch nicht signifikant $(p=0,308)$.

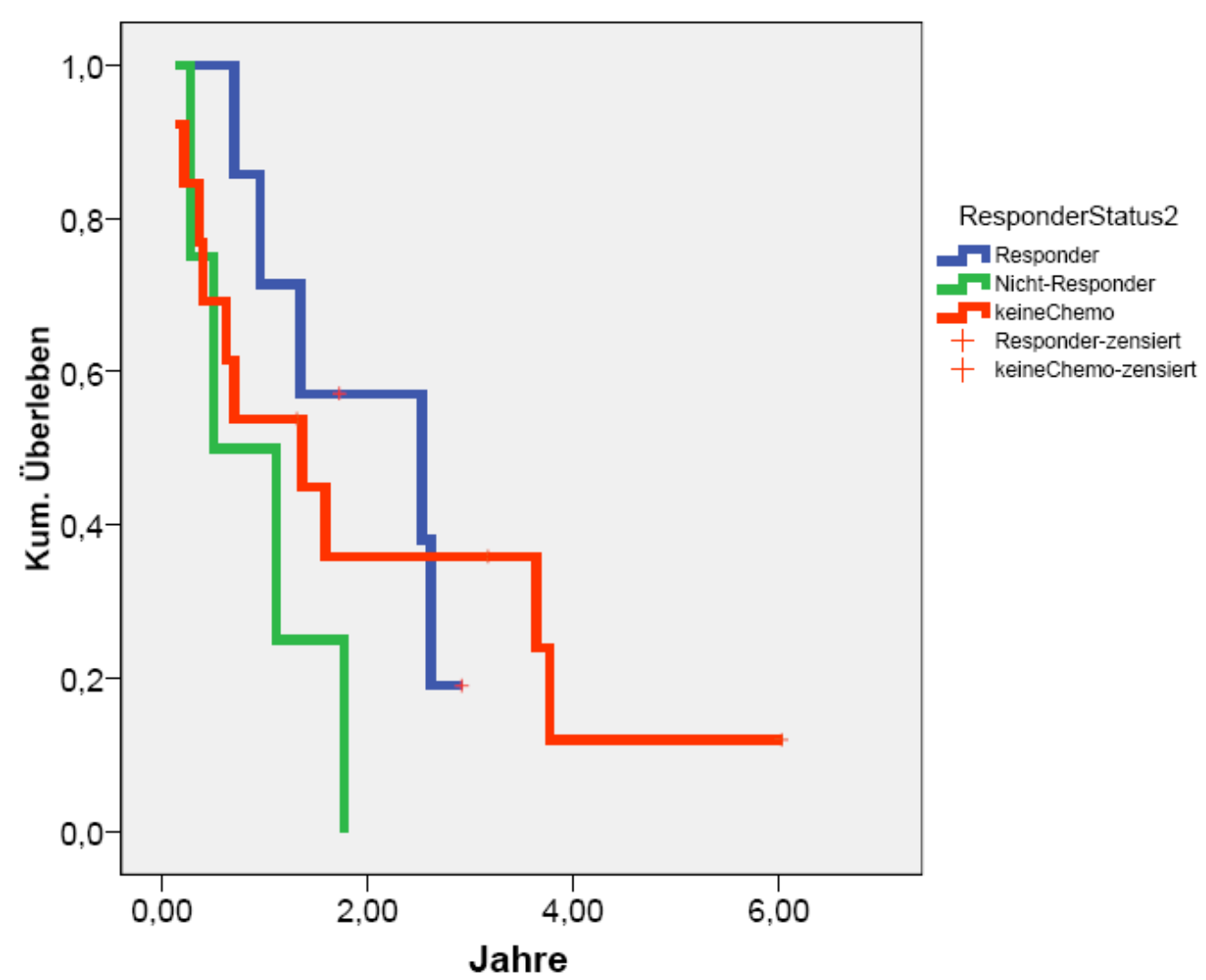

Abb. 14 Überlebensraten bei spätem Tumorstadium, UICC III/IV, postoperativ 


\subsubsection{Resektionsstatus}

Nach kurativer Resektion hatten die Responder ( $n=17 / 20$ ) eine 5-JÜR von 49,2\%. Das mediane Überleben lag bei 2,6 Jahren.

Nicht-Responder ( $n=3 / 6)$ hatten eine 5 -JÜR von $50 \%$ bei einem medianen Überleben von 1,1 Jahren.

Bei den nur Operierten ( $n=28 / 38$ ) lag die 5-JÜR nach R0-Resektion bei 53,3\%.

Der Unterschied in der Überlebensprognose war statistisch nicht signifikant $(p=0,879)$.

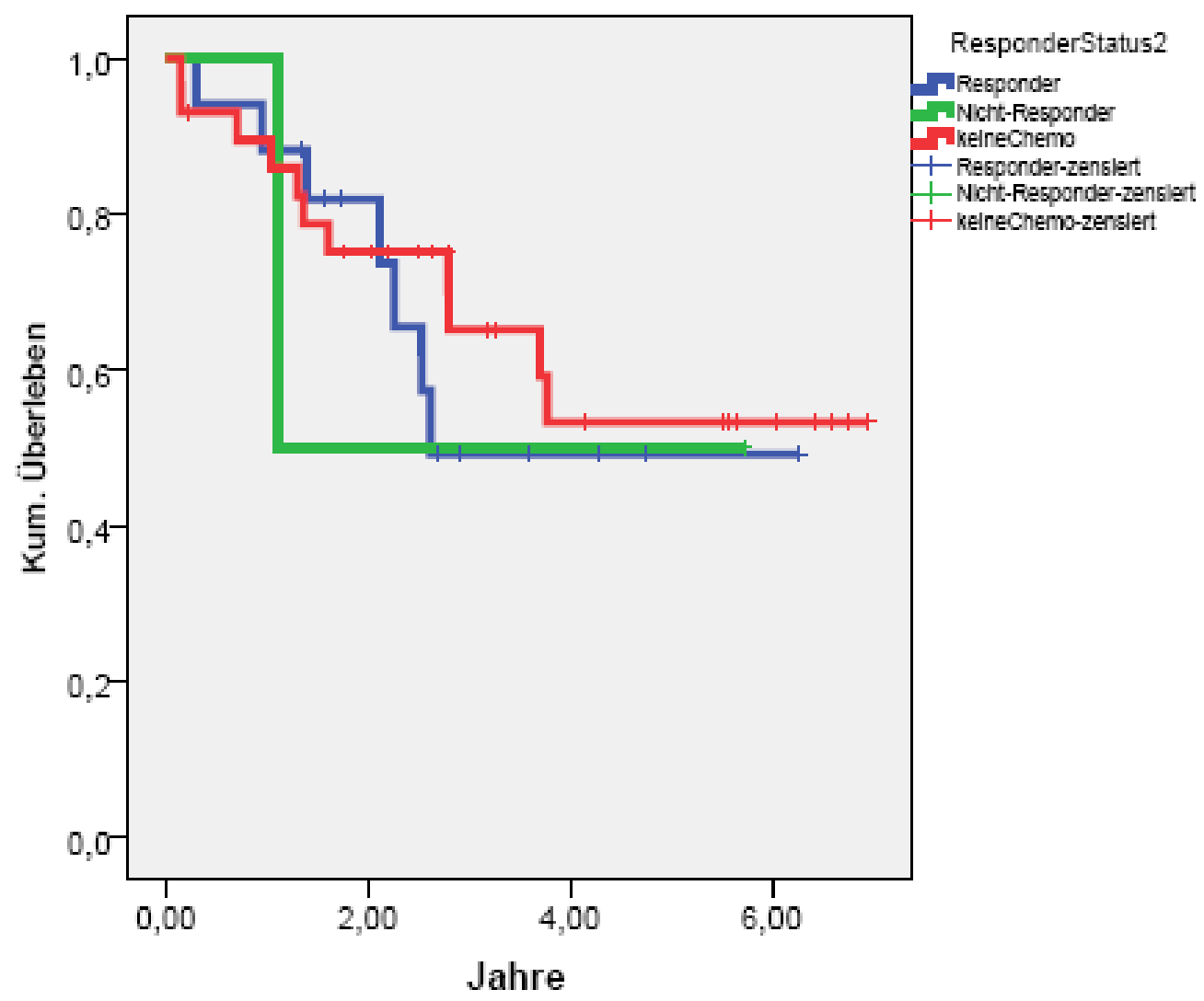

Abb. 15 Überlebensraten bei R0-Resektion 
Nach palliativer Resektion lag die 5-JÜR der Responder bei 33,3\% ( $n=3 / 20)$. Das mediane Überleben lag bei 1,3 Jahren bei einer Streubreite von 0,3 bis 2,4 Jahren.

Von den R+-resezierte Nicht-Respondern ( $n=3 / 6$ ) überlebte kein Patient 5 Jahre. Das mediane Überleben lag bei 0,5 Jahren bei einer Streubreite von 0,1 bis 0,8 Jahren.

Von den R+-resezierten Patienten der nur Operierten ( $n=10 / 38)$ hat kein Patient 5 Jahre überlebt. Das mediane Überleben lag bei 0,4 Jahren bei einer Streubreite von 0,1 bis 0,8 Jahren.

Der Unterschied in der Überlebensprognose war statistisch nicht signifikant $(p=0,761)$.

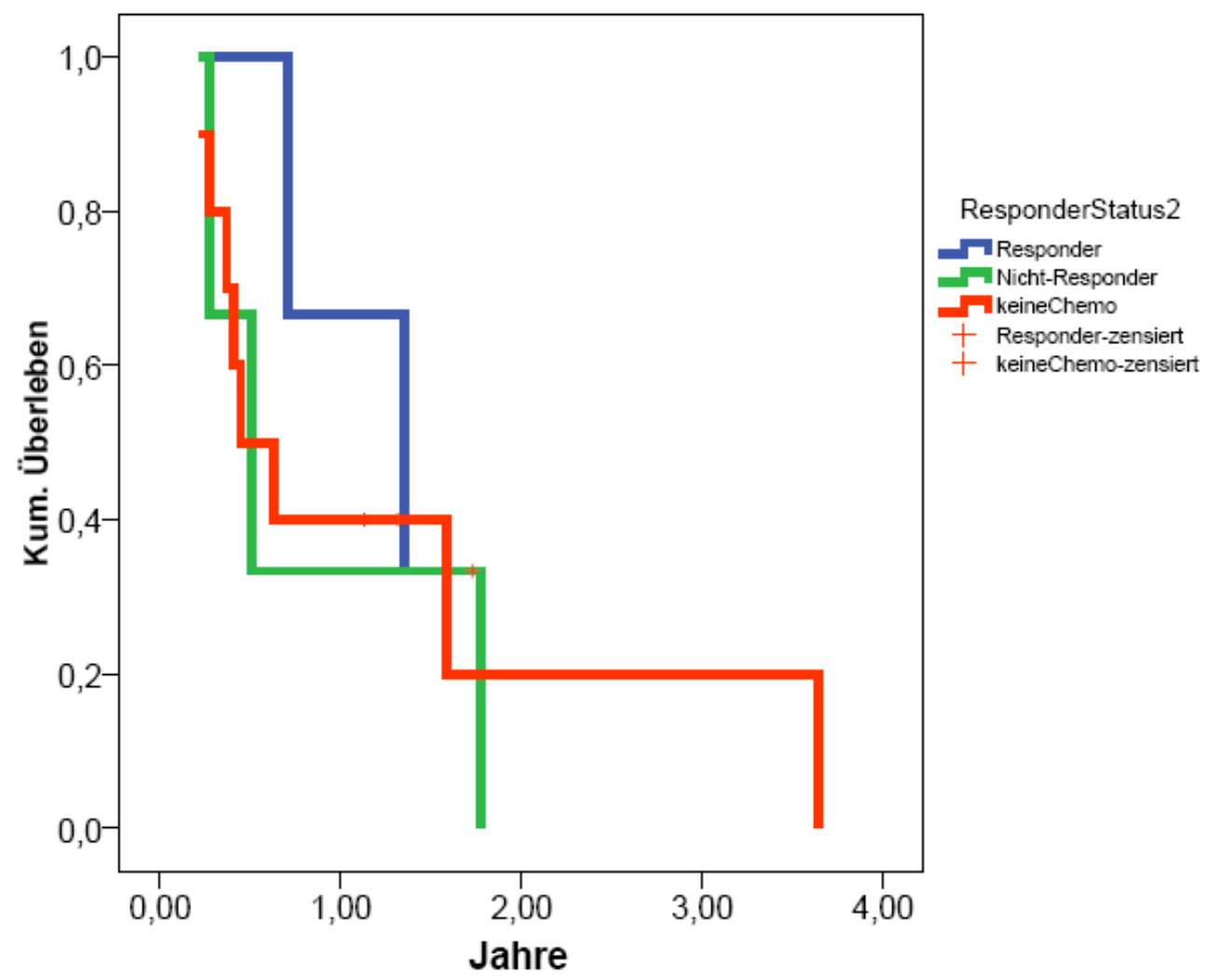

Abb. 16 Überlebensraten bei R+-Resektion 


\subsubsection{Resektionsstatus und Fernmetastasen}

Nach R0-Resektion und ohne Fernmetastasen hatten die Responder ( $n=17 / 20)$ eine 5-JÜR von $49,2 \%$. Das mediane Überleben lag bei 2,6 Jahren.

Nicht-Responder hatten nach kurativer Resektion und ohne Fernmetastasen ( $n=3 / 6)$ eine 5-JÜR von 50\%. Das mediane Überleben lag bei 1,1 Jahren.

Bei den nur Operierten ( $n=29 / 38)$ lag die 5-JÜR bei 53,3\%.

Der Unterschied in der Überlebensprognose war statistisch nicht signifikant $(p=0,879)$.

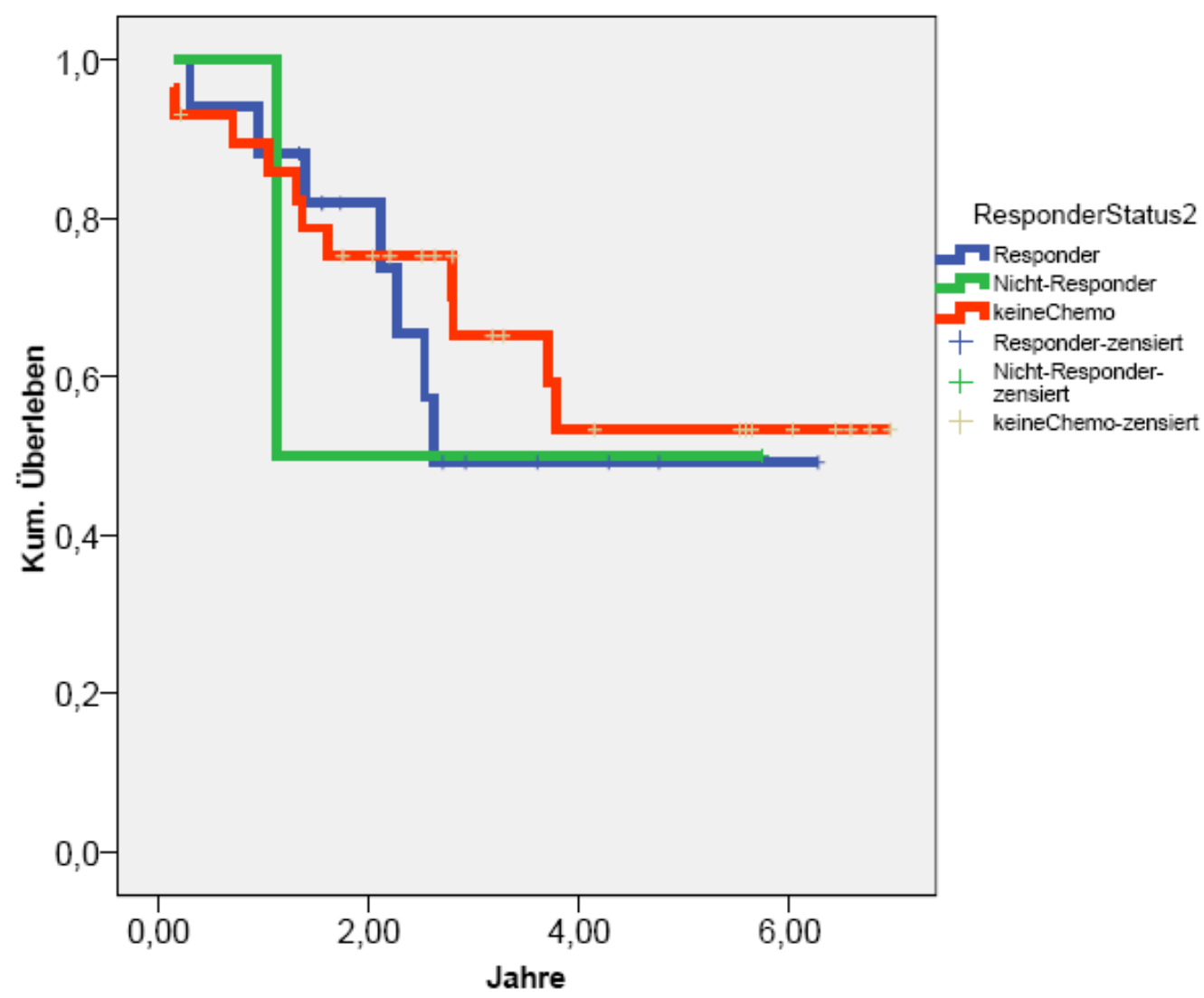

Abb. 17 Überlebensraten bei R0-Resektion ohne Fernmetastasen (M0) 
Nach R1-Resektion und ohne Fernmetastasen hatten Responder $(n=3 / 20)$ eine 5JÜR von $50 \%$. Das mediane Überleben lag bei 1,4 Jahren.

Von den Nicht-Respondern $(n=1 / 6)$ hat kein Patient 5 Jahre überlebt. Das mediane Überleben lag bei 1,7 Jahren.

Von den nur Operierten $(n=6 / 38)$ hat kein Patienten 5 Jahre überlebt. Das mediane Überleben lag bei 0,6 Jahren bei einer Streubreite von 0,0 bis 2,5 Jahren.

Der Unterschied in der Überlebensprognose erwies sich als statistisch nicht signifikant $(p=0,933)$.

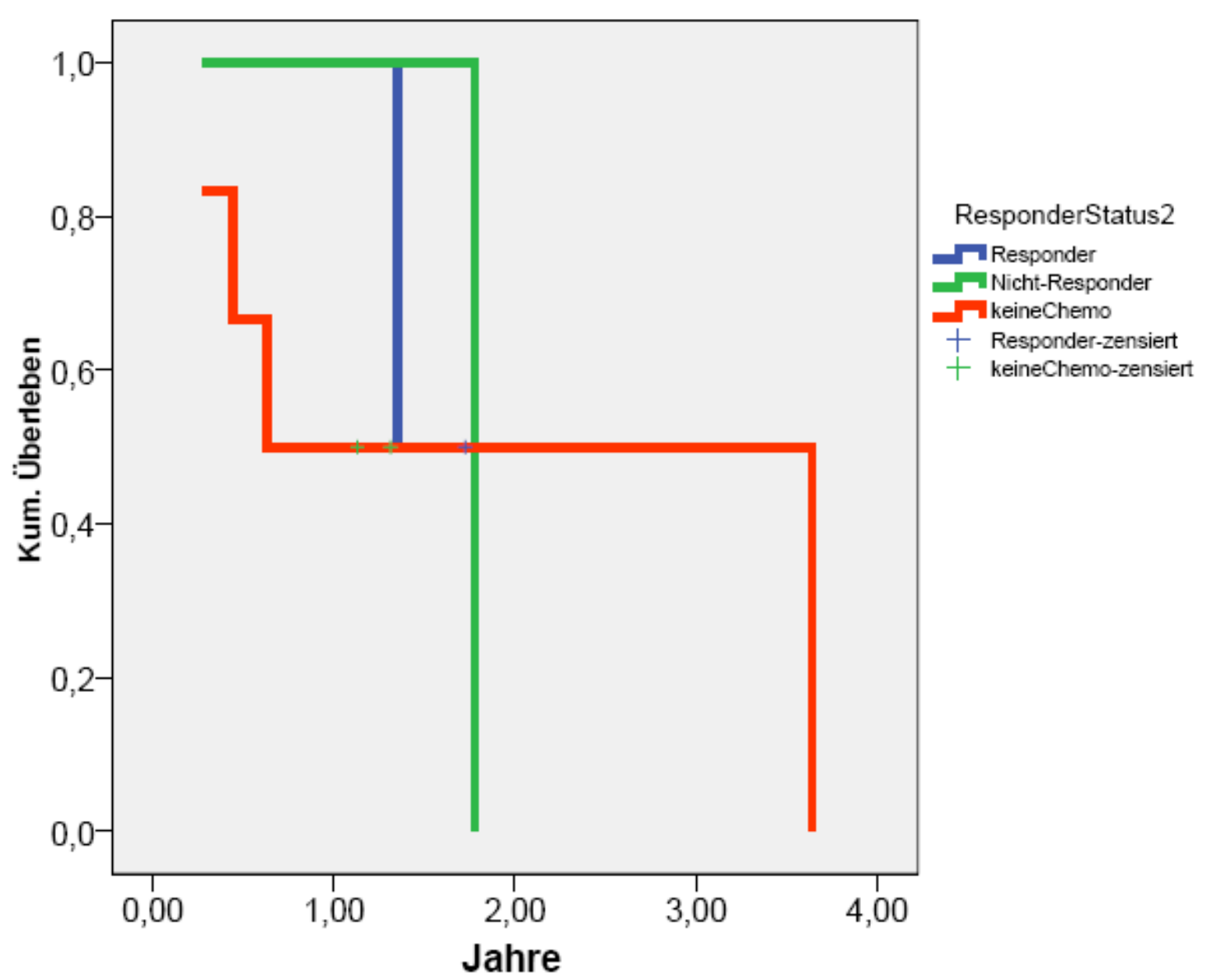

Abb. 18 Überlebensraten bei R+-Resektion ohne Fernmetastasen 


\subsubsection{Fernmetastasen}

Unabhängig vom Resektionsstatus lag die 5-JÜR der Responder ohne Fernmetastasen ( $n=19 / 20)$ bei 47\%. Das mediane Überleben bei 2,6 Jahren.

Nicht-Responder ohne Fernmetastase $(n=4 / 6)$ hatten eine 5-JÜR von 33,3\%. Das mediane Überleben lag bei 1,7 Jahren bei einer Streubreite von 0,7 bis 2,8 Jahren.

Nur Operierte ohne Fernmetastasen ( $n=35 / 38$ ) hatten eine 5-JÜR von 46\%, bei einem medianen Überleben von 3,7 Jahren.

Der Unterschied im Überleben war statistisch nicht signifikant $(p=0,825)$.

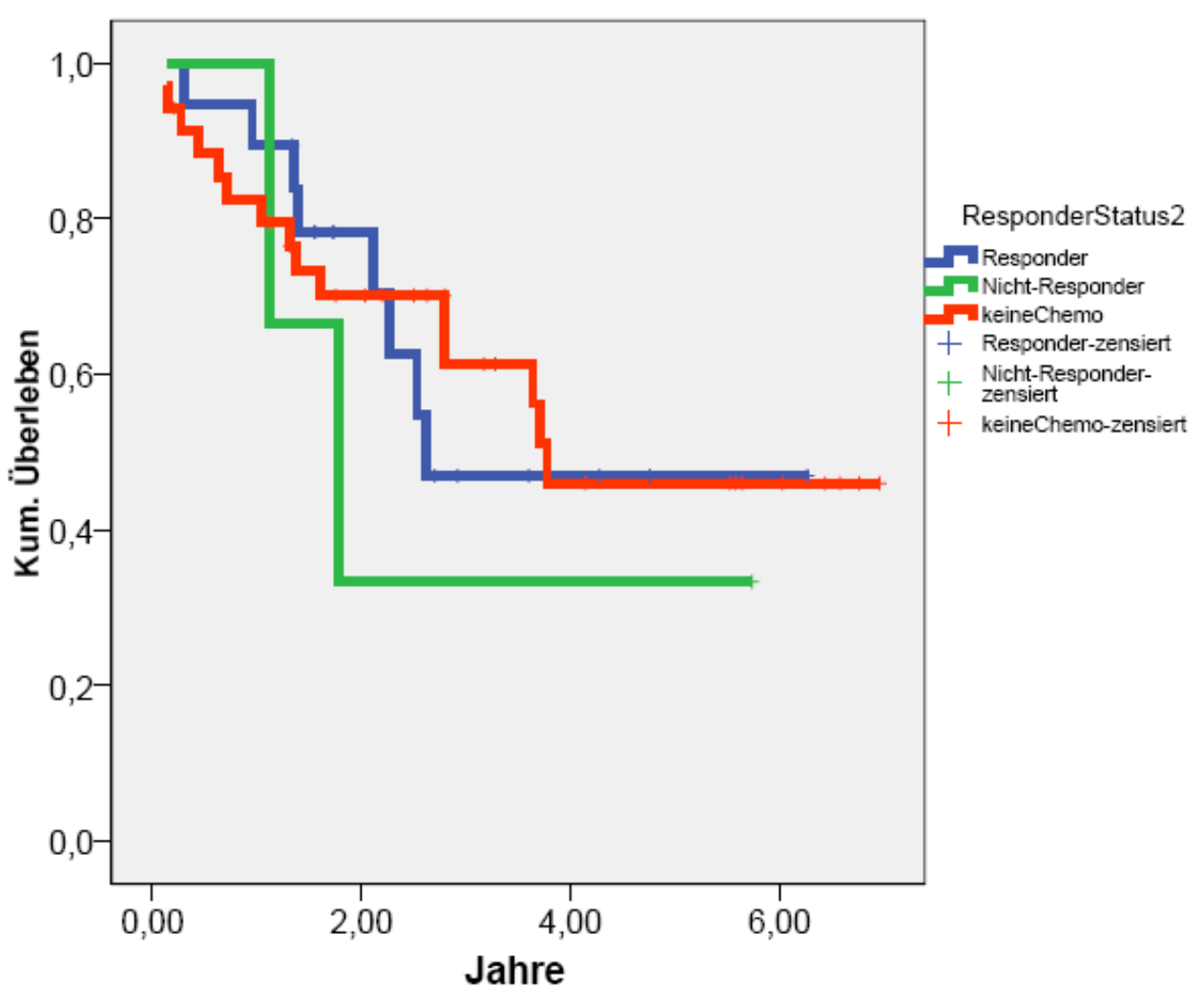

Abb. 19 Überlebensraten ohne Fernmetastasen (M0) 
Mit Fernmetastasen hat kein Patient der Responder $(n=1 / 20) 5$ Jahre überlebt. Das mediane Überleben lag bei 0,7 Jahren.

Von den Nicht-Respondern $(n=2 / 6)$ hat kein Patient 5 Jahre überlebt. Das mediane Überleben lag bei 0,3 Jahren.

Nur Operierte mit Fernmetastasen ( $n=3 / 38$ ) haben keine 5 Jahre überlebt. Das mediane Überleben lag bei 0,4 Jahren.

Der Unterschied in der Überlebensprognose erwies sich statistisch als nicht signifikant $(p=0,768)$.

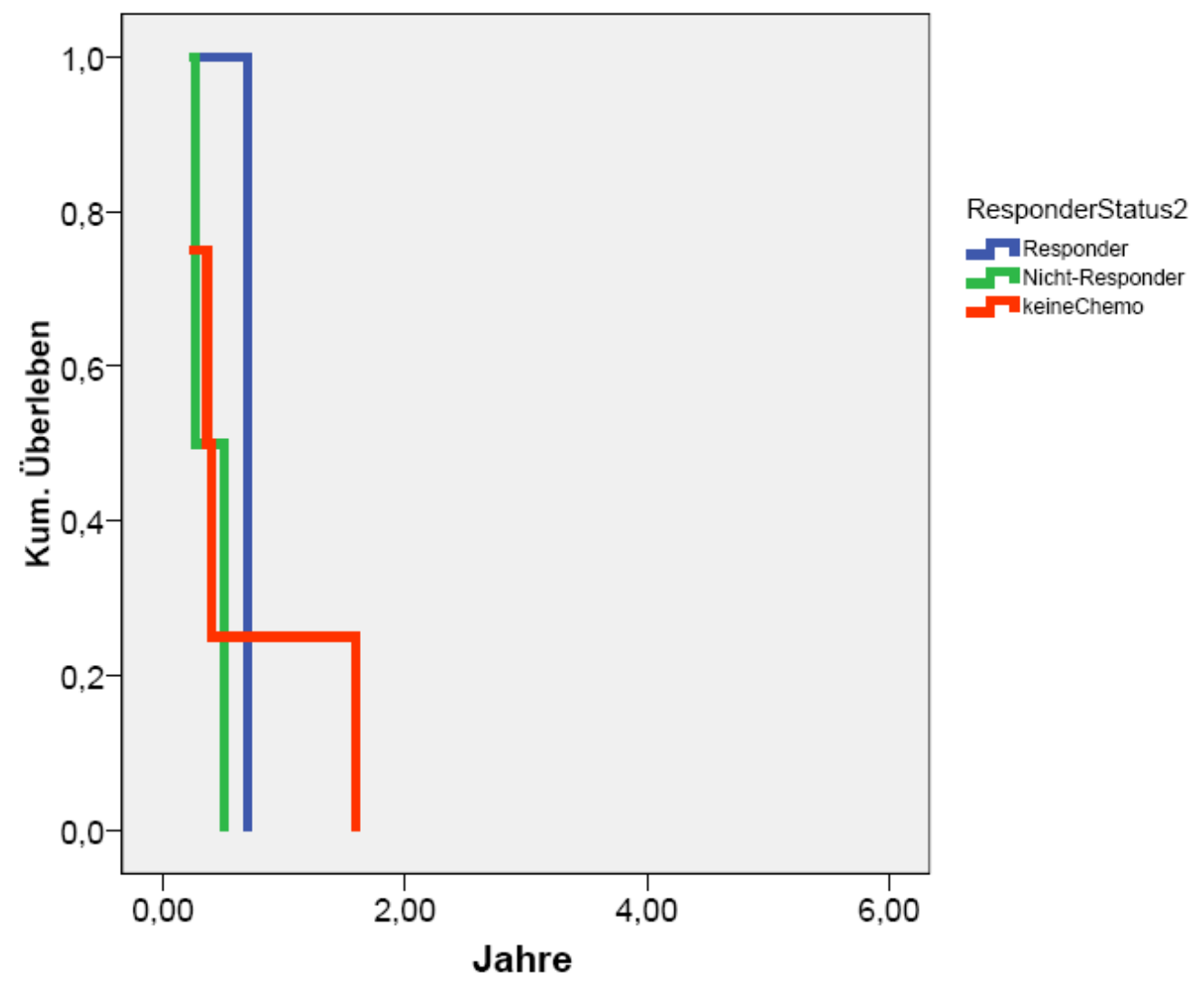

Abb. 20 Überlebensraten mit Fernmetastasen (M+) 


\subsubsection{Lymphknotenmetastasen}

Ohne Lymphknotenmetastasen hatten Responder ( $n=8 / 20)$ eine 5-JÜR von 75\%.

Von den Nicht-Respondern $(n=2 / 6)$ hat kein Patient 5 Jahre überlebt. Das mediane Überleben lag bei 0,3 Jahren.

Nur Operierte $(n=17 / 38)$ hatten eine 5-JÜR von 64,5\%.

Der Unterschied in der Überlebensprognose war statistisch signifikant $(p=0,003)$.

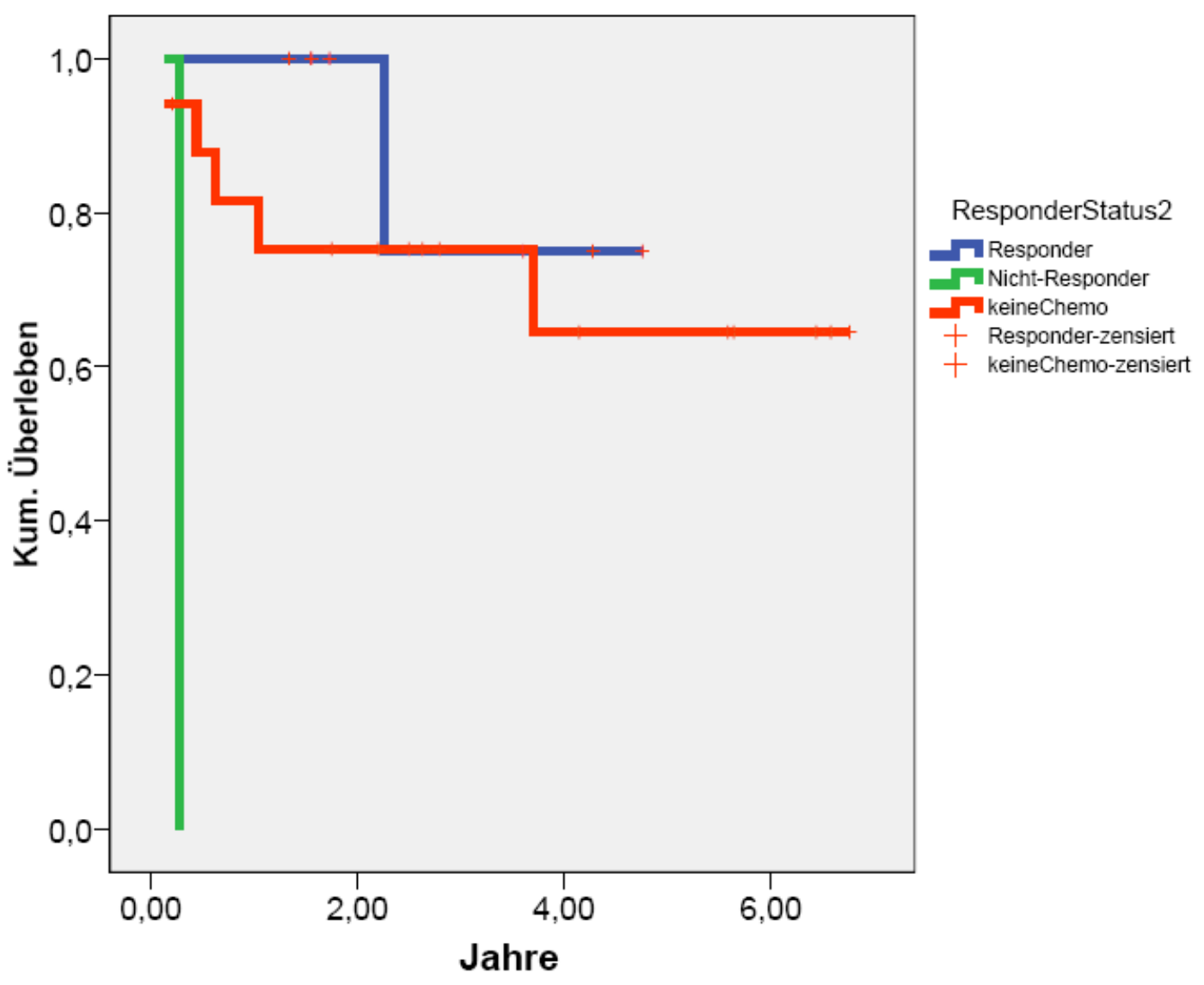

Abb. 21 Überlebensraten ohne Lymphknotenmetastasen 
Mit Lymphknotenmetastasen hatten Responder ( $n=12 / 20)$ eine 5-JÜR von 29,2\%. Das mediane Überleben lag bei 2,1 Jahren bei einer Streubreite von 0,4 bis 3,8 Jahren.

Nicht-Responder ( $n=4 / 6$ ) hatten eine 5-JÜR von 25,0\%. Das mediane Überleben lag bei 1,1 Jahren bei einer Streubreite von 0,0 bis 2,4 Jahren.

Bei den nur Operierten ( $n=21 / 38$ ) lag die 5-JÜR bei 24,4\%. Das mediane Überleben lag bei 2,8 Jahren bei einer Streubreite von 1,2 bis 4,4 Jahren.

Der Unterschied in der Überlebensprognose war statistisch nicht signifikant $(p=0,94)$.

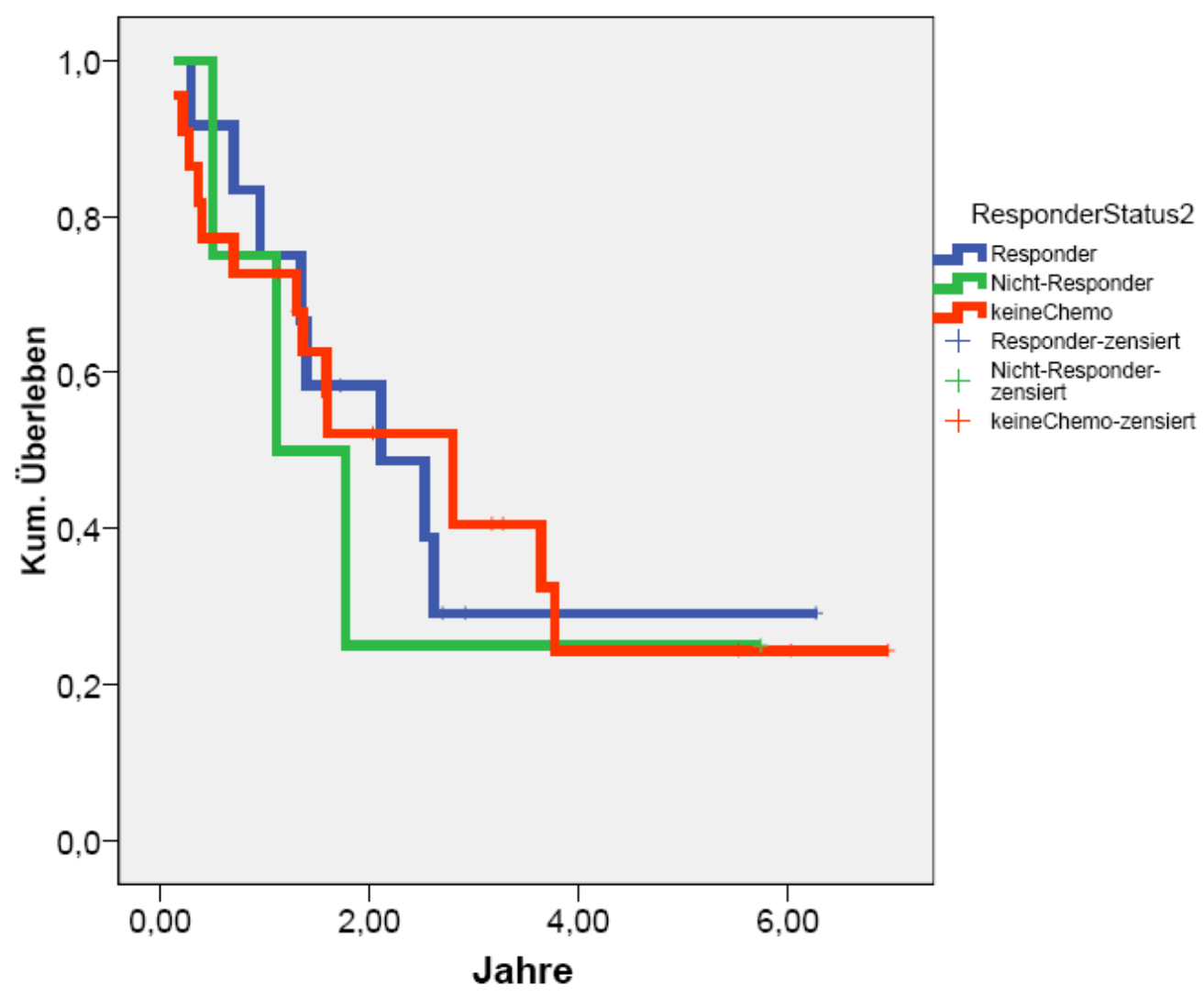

Abb. 22 Überlebensraten mit Lymphknotenmetastasen 


\section{Diskussion}

Die Prognose des Magenkarzinoms hat sich in den vergangenen 15 Jahren nicht verändert. Die 5-JÜR liegt bei 40-50\%. Durch die intraoperative Radiatio (IORT) konnte die lokale Rezidivrate gesenkt werden, die Gesamtprognose wurde jedoch nicht beeinflusst [14]. Mit einer palliativen Chemotherapie konnte bei fortgeschrittenem Magenkarzinom eine signifikante Verbesserung des medianen Überlebens erzielt werden. Daher wurde in einer Studie geprüft, ob durch ein neoadjuvantes Setting die Prognose des Magenkarzinoms positiv beeinflusst werden kann.

In die vorliegende Studie wurden bei retrospektiver Auswertung der Daten insgesamt 64 Patienten aufgenommen; sie liegt damit im unteren Drittel derzeitiger Literaturdaten [11,14,21,33,37,39].

Hinsichtlich der Alters- und Geschlechtsverteilung mit 69 Jahren im Durchschnitt und einem Überwiegen des männlichen Geschlechts sowie der beschriebenen Symptome, den Verfahren zur Diagnosestellung und zum Staging stimmte unsere Studie mit vergleichbaren überein [28,56,57]. Ebenso waren die Ergebnisse unserer Studie bezüglich Tumorlokalisation (71,9\% proximal), WHO-Klassifikation $(79,7 \%$ Adenokarzinoms) und bezüglich dem häufigen Auftreten undifferenzierter Karzinome $(57,7 \%)$ mit den Ergebnissen anderer vergleichbar [11,21,28,33,37,39,56,57]. Unterschiede gab es hinsichtlich der histopathologisch eruierten LaurenKlassifikation bei $56,3 \%$ für den intestinalen Typ gegenüber $46,7 \%$ in anderen Studien. Hier überwog der diffuse Typ [21,23].

Die Einschlusskriterien wie Diagnose eines Magenkarzinoms ohne eine andere weitere Tumorlokalisation (Ösophagus), fortgeschrittenes Tumorstadium (UICC Ib-III) mit Ausschluss einer Peritonealkarzinose und/oder Lebermetastasen, gute Nierenund Leberfunktion sowie keine weitere Malignomerkrankung stimmten mit anderen Studien überein $[21,33,39]$. Geringe Abweichung bestand in der Auswahl der einzelnen UICC-Stadien: in die vorliegende wie auch in eine vergleichbare Untersuchung wurden Stadien Ib-III eingeschlossen, in anderen Studien hingegen nur Stadium IV oder M+ oder ausschließlich die Stadien IIla/b und IV [21,33,39]. 
In unserer Studie lagen prätherapeutisch bei $81,3 \%$ fortgeschrittene Tumorstadien (UICCII/III) vor. Diese Verteilung entsprach der vergleichbarer Studien $[21,23,33,37,43] .19 \%$ hatten Stadium Ib.

Wegen der fortgeschrittenen Tumore wurde in der vorliegenden Studie wie in vergleichbaren Arbeiten bei der Mehrzahl der Patienten (87,5\%) eine totale oder erweiterte Gastrektomie in Kombination mit der systematischen Lymphadenektomie (D2-LAD) durchgeführt; die D1-LAD und die subtotale Gastrektomie erfolgten bei frühen Tumorstadien und der Histologie des intestinalen Typs nach Lauren in 17,2\% $[21,43,48,53,57]$.

Im Gesamtkollektiv lag die R0-Resektionsrate bei 75\% ( $n=48 / 64)$; sie war höher als die in anderen Studien erreichten Ergebnisse [19,21,33,39]. In dieser Studie wurde bei den nur operierten Patienten das tatsächliche postoperativ diagnostizierte Tumorstadium beim prätherapeutischen Staging überschätzt: nur 7 der 15 bildmorphologisch eruierten UICC III Stadien wurden postoperativ bestätigt. Eine sichere Unterscheidung der Tumorstadien insbesondere Ib, II und III war nicht möglich. Bei Operation lagen vermutlich mehr frühere Stadien vor. Nach Chemotherapie wird der Vergleich zum postoperativen Stadium zusätzlich unsicher, da das therapiebedingte Downstaging in das postoperative Stadium bei der Beurteilung der Bildgebung mit eingeht. Ähnliche Ergebnisse wurden bisher in der Literatur nicht beschrieben und sind bei der Einschätzung der bisher mitgeteilten Studienergebnisse zu berücksichtigen [21,33,39].

Postoperative Komplikationen traten im Gesamtkollektiv bei $54,6 \%$ auf. Nichtchirurgische Komplikationen entsprachen den Beobachtungen anderer Arbeiten [36,44]. Chirurgische Komplikationen wie Wundheilungsstörungen, verzögerter Kostaufbau und lleus waren nicht häufiger als in anderen Studien und auch die Anastomoseninsuffizienz mit 10,9\% im Gesamtkollektiv entsprach bereits berichteten Ergebnissen [14,21,39].

Die Rezidivrate des Gesamtkollektivs mit 51,6\% war vergleichbar mit den in der Literatur berichteten Rezidiven von 20-53\% bzw. 69\% [21,27,31,37,39].

Das lokoregionäre Rezidiv kam in der vorliegenden Studie in $20,3 \%$ vor und war deutlich niedriger als die aktuell berichtete Lokalrezidivrate von $87 \%$ [31]. Die 
Häufigkeit von Peritonealkarzinose (10,9\%), Lebermetastasen (7,8\%) oder Lymphknotenmetastasen (7,8\%) entsprach vergleichbaren Studien [39].

Eine neoadjuvante Chemotherapie nach PELF erhielten in dieser Studie 26/64 Patienten therapiert. 69,2\% erhielten die vollständigen 2 Zyklen; dies waren weniger als in anderen Studien mit 95\% und 90\% [21,39]. Zeichen der Toxizität wie Neutropenie, Knochenmarksdepression, Übelkeit oder Erbrechen sowie Tumorprogress führten wie in anderen Arbeiten zum vorzeitigen Abbruch $[21,39]$.

Vor Beginn der Chemotherapie (CTx) und nach dem jeweils letzten Zyklus (Restaging) wurden wie in vergleichbaren Arbeiten die Tumorstadien mittels Endoskopie, CT und Endosonographie ermittelt [21,33,39]. In beiden Studiengruppen hatten prätherapeutisch die meisten Patienten fortgeschrittene Karzinome: 92,3\% der Chemotherapierten und 74\% der nur Operierten hatten UICC-Stadium II/III. Dies Iag im Bereich der von vergleichbaren Studien berichteten Häufigkeit fortgeschrittener Stadien von $36 \%$, 51\% und 90\% [14,21,43].

Hinsichtlich der Beurteilung des Response im Rahmen des Restagings gab es Abweichung zur aktuellen Literatur: in dieser Untersuchung wurden die Karzinome beim Restaging erneut nach UICC klassifiziert, um ein Downstaging nachvollziehen zu können, während in vergleichbaren Studien der Therapieeffekt ausschließlich postoperativ als kompletter oder partieller Response beschrieben wurde [21,39].

Postoperativ konnte ein Ansprechen objektiv beurteilt werden; histopathologisch zeigten in dieser Studie 20/26 Patienten einen Response (76,9\%). Die Responserate war höher als die einer anderen Studie mit neoadjuvanter Chemotherapie nach PELF von 36,8\% $(n=21 / 57)$ [21]. Auch die mit anderen Chemotherapie-Schemata erreichten Responseraten (FAM-, ECF- bzw. FLEP-Schema) von 30\% bis 50\% lagen unter dem Ergebnis der vorliegenden Studie [11,39,52,]. Die 20 Responder hatten histopathologisch unterschiedliche Regressionsgrade: 5 (25\%) hatten einen kompletten Response (CR; pT0/1N0) und 15 (75\%) zeigten einen partiellen Response (PR). In vergleichbaren Studien kam ein CR mit einem Patienten wesentlich seltener vor und die PR-Rate war mit 33-50\% niedriger als die der vorliegenden Studie [21,39]. 
Ein gutes Ansprechen wurde in dieser Studie wie auch in anderen insbesondere im UICC-Stadium III beobachtet: 4mal kam ein kompletter und 9mal ein partieller Response vor. im Stadium Ib/II waren sowohl ein kompletter wie ein partieller Response weniger häufig [14,21,33]. Die Responserate des fortgeschrittenen Stadiums (UICCIII) von 76\% lag im Bereich der in anderen Studien beobachteten Responseraten von $57,9 \%$ bzw. 74,1\% für Stadium III [21,39].

Das Ansprechen auf die CTx wird durch das zu beobachtende Downstaging dokumentiert. Nach Chemotherapie waren niedrigere Tumorstadien häufiger zu beobachten: postoperativ wurden die Stadien I und II (UICC) 18mal (69,2\%) im Gegensatz zu prätherapeutisch 9mal (34,6\%) diagnostiziert; weiterhin hatte die Häufigkeit von Stadium III von ursprünglich 17 (65,3\%) auf 3 (11,5\%) abgenommen $[11,21,39]$.

23,1\% der Patienten ( $n=6 / 26)$ unserer Studie sprachen nicht auf die Chemotherapie an; eine vergleichbare Studie hatte mit 8/30 Nicht-Respondern (26,6\%) ein ähnliches Ergebnis [39]. Mit 47,4\% $(n=27 / 57)$ hatte eine andere Studie mehr Nicht-Responder als unsere Studie [21].

Ein Tumorprogress und damit ein negativer Effekt der Chemotherapie wurde in unserer Studie in 2 Fällen beobachtet; häufiger war eine Konstanz des Karzinomstadiums ( $n=4 / 6)$. In anderen Studien war ein Tumorprogress bei 9/57 bzw. 7/30 Patienten etwas weniger häufig festzustellen [21,39].

Die R0-Resektionsrate nach Chemotherapie lag bei 76,9\%. Gegenüber den nur Operierten mit 73,7\% unterschied sie sich nicht signifikant, war jedoch höher als die in der aktuellen Literatur beschriebenen R0-Resektionsraten von 17\% bzw. 47\% bei Responseraten von 37\% bzw. 50\% [21,39].

Die ähnlichen R0-Resektionsraten unserer Studiengruppen legen die Vermutung nahe, dass auch bei den Chemotherapierten eine Unsicherheit im tatsächlichen Tumorstadium zu Beginn der Therapie besteht. Dies kann aufgrund des Downstagings in der Histologie nicht nachvollzogen werden.

Die Morbidität und auch das Vorkommen der Anastomoseninsuffizienz $(11,5 \%)$ waren nach neoadjuvanter Chemotherapie nicht erhöht. Nach Operation hatten 
$10,5 \%$ eine Insuffizienz an der Anastomose und vergleichbare Arbeiten beobachteten nach Chemotherapie Komplikationsraten von 16,7\%-45\% und Insuffizienzraten von $20-33,3 \%[11,21,39]$.

Nach CTx lag die Rezidivrate bei 38,4\% und war günstiger als die der nur Operierten von 60,5\%; nach IORT bzw. Chemotherapie waren die Rezidivraten mit $61 \%$ bzw. $67 \%$ höher [14,39]. In einer anderen Arbeit wurde über eine reduzierte Rezidivrate allerdings nach adjuvanter Chemotherapie berichtet [33].

In unserer Studie war die Lokalrezidivrate nach neoadjuvanter CTx mit 11,5\% gegenüber den nur Operierten mit 23,3\% verringert. Sie lag im Bereich der Lokalrezidivrate nach IORT von 9,8\% [14].

Eine Peritonealkarzinose war nach Therapie In der vorliegenden Untersuchung in beiden Gruppen ähnlich häufig, $11,5 \%$ in Gruppe 1 und 19,5\% in Gruppe 2, und konnte durch die CTx nicht verhindert werden. Lebermetastasen waren nach CTx mit 9,4\% seltener aufgetreten als in vergleichbaren Arbeiten mit 36,8\% [37].

Rezidive in Form von Lymphknotenmetastasen kamen in unserer Studie nur in Gruppe 2 vor ( $n=5 / 30)$ - was mit dem in ähnlichen Arbeiten beobachteten Ansprechen von Tumorzellen, Mikrometastasen, besonders in Lymphknoten erklärbar sein könnte $[11,39]$.

Die Prognose des Magenkarzinoms konnte in dieser Studie durch neoadjuvante Chemotherapie nicht günstig beeinflusst werden. Nach CTx lag die 5-JÜR bei 37,5\% und bei den nur Operierten bei $42,3 \%$. Vergleichbare Studien beobachteten ebenfalls keinen signifikanten Überlebensvorteil nach CTx [21,39].

Die aktuelle Studienlage sieht das histologische Ansprechen auf eine CTx als einen wichtigen prognostischen Faktor [19,21,39]. Die Subgruppenanalyse zeigte, dass in dieser Studie trotz der hohen Responserate (76,9\%) das Überleben der Responder gegenüber den nur Operierten nicht verbessert worden war. Responder hatten eine 5-JÜR von $44,6 \%$ und unterschieden sich nicht signifikant von der 5-JÜR der nur Operierten von $42,3 \%$. Von den Nicht:-Respondern überlebte ein Patient 5 Jahre. in vergleichbaren Studien konnte ebenfalls kein Prognosevorteil für Responder beobachtet werden $[19,21,39]$. 
im UICC-Stadium III konnte die Subgruppenanalyse einen signifikanten Überlebensvorteil für Responder gegenüber nur Operierten zeigen; die 5-JÜR lagen bei 45,7\% bzw. 31,1\%. Nicht-Responder erreichten die 5-Jahresgrenze nicht. In frühen Tumorstadien, UICC Ib/Il, hatten Responder keinen Prognosevorteil gegenüber den nur Operierten (5-JÜR 42,9\% bzw. 48,6\%). Nicht-Responder verhielten sich wie nur Operierte.

Die neoadjuvante CTx sollte die Prognose fortgeschrittener Tumoren durch eine Eindämmung der Tumorzellausbreitung insbesondere in Lymphknoten beeinflussen [19]. Lymphknotenmetastasen waren bei Chemotherapierten nach 5 Jahren nicht zu beobachten, das Überleben der Responder ohne Lymphknotenmetastasen unterschied sich jedoch nicht signifikant von dem der nur Operierten; die 5-JÜR lagen bei $75 \%$ und $64,5 \%$. Von den Nicht-Respondern überlebte keiner 5 Jahre. Mit Lymphknotenmetastasen war die Prognose der Responder mit einer 5-JÜR von $29,2 \%$ auch nicht signifikant besser als die der nur Operierten und Nicht-Responder von $24,4 \%$ und $25,5 \%$. Ein Überlebensvorteil bei Lymphknotenmetastasen konnte in einer ähnlichen Studie mit adjuvanter CTx beobachtet werden: die 5-JÜR lag bei $42 \%$ nach CTx versus $22 \%$ für nur operierte Patienten [54].

Ein weiterer Effekt der CTx sollte die Therapie okkulter Mikrometastasen sein und dadurch ein längeres rezidivfreies Überleben ermöglicht werden $[21,27]$. Doch trotz der in dieser Studie beobachteten Reduktion des Lokalrezidivs wurde das Überleben (37,5\%) durch die CTx nicht verbessert. Mit Rezidiv überlebte kein Patient nach CTx 5 Jahre, während die nur Operierten eine 5-JÜR von 31\% hatten. Ohne Rezidiv hatten die nur Operierten ein signifikant besseres Überleben mit einer 5-JÜR von 83\% gegenüber den Respondern mit einer 5-JÜR von 55\%.

In der Subgruppe der palliativ Resezierten (R1) zeigte sich für Responder gegenüber den nur Operierten ein, wenn auch nicht signifikanter Überlebensvorteil; mit einer 5JÜR von 33,3\% war ihre Prognose gegenüber den nur Operierten, von denen keiner die 5-Jahresgrenze erreichte, günstiger. Das mediane Überleben der Responder unserer Studie von 15 Monaten nach R1-Resektion lag im Bereich vergleichbarer Studien, die von einem medianen Überleben von 11-13 Monaten berichteten [39]. Von den Nicht-Respondern überlebte keiner 5 Jahre. 
Durch Erhöhung der Wahrscheinlichkeit auf kurative Resektion sollte durch neoadjuvante CTx vor allem bei fortgeschrittenem Stadium (>UICC III) die Prognose günstig beeinflusst werden [19]. In dieser Studie konnte für Responder nach kurativer Resektion bei einer Rate von 76,9\% kein signifikanter Überlebensvorteil gegenüber den nur Operierten gezeigt werden; die 5-JÜR lagen bei 49,2\% für Responder und 53,3\% nach alleiniger Chirurgie, wobei die R0-Resektionsrate der nur Operierten mit $71,7 \%(n=27 / 38)$ ähnlich hoch war. Nicht-Responder erreichten die 5-Jahresgrenze nicht.

Bisher wurde nur in einer Studie ein Überlebensvorteil für die Chemotherapie-Gruppe mit einer 5 -JÜR von $35 \%$ vs. $23 \%$ nach alleiniger Chirurgie beobachtet, war allerdings nicht günstiger als das bisher erreichte Gesamtüberleben $[11,19,39]$.

Wie auch diese Pilotstudie zeigen konnte, handelt es sich beim fortgeschrittenen Magenkarzinom um eine systemische Erkrankung. Bedeutendes prognostisches Kriterium für das Magenkarzinom ist das intraperitoneale und lokale Rezidiv. Durch die neoadjuvante Chemotherapie, wie in dieser Studie gezeigt, wird zwar das lokale Rezidiv positiv beeinflusst, jedoch nicht das Gesamtüberleben. Die Kombination einer lokalen Maßnahme mit einer systemischen Therapie wie Chemotherapie in Kombination mit Antikörpern oder weitere CTx als adjuvante Langzeittherapie, ähnlich wie beim Rektumkarzinom, könnte einen günstigen Einfluss erwarten lassen. 


\section{Zusammenfassung}

In einer retrospektiven Studie wurde bei Patienten mit Magenkarzinom, die von 2000 bis 2006 in der Chirurgischen Universitätsklinik Freiburg behandelt wurden, der Einfluss einer neoadjuvanten Chemotherapie auf das Downstaging, die Morbidität und die Prognose untersucht.

Entsprechend dem präoperativen Staging mit Endoskopie, Endosonographie, CT und Laparaskopie wurden 64 Patienten mit fortgeschrittenen Tumorstadien (UICC lbIII) in die Studie eingeschlossen. 38 Patienten wurden nur operiert, 26mal konnte eine neoadjuvante Chemotherapie durchgeführt werden, die mit Cisplatin, Epirubicin, Leukovorin und 5-FU (PELF) erfolgte. Anschließend wurden die Patienten operiert. 56/64 Patienten erhielten eine totale und 8 eine subtotale Gastrektomie. 61/64 Patienten wurden erweitert lymphadenektomiert (D2). Die R0-Resektionsrate lag bei 48/64 Patienten; nach Chemotherapie wurden 20/26 R0-reseziert, bei den nur Operierten 28/38 Patienten.

Die Chemotherapie erfolgte bei 18/26 Patienten in 2 Zyklen, 6 mal musste nach dem ersten Zyklus wegen Toxizität abgebrochen werden, einmal wurden ein Tumorprogress und einmal eine Vollremission festgestellt. 15/20 Patienten zeigten histologisch einen partiellen, 5 einen kompletten Response; die Responserate lag bei $76,9 \%$ ( $n=20 / 26$ ). Dabei sprachen höhere Tumorstadien (UICCIII) besser auf die Chemotherapie an. Die perioperative Morbidität bezüglich Anastomoseninsuffizienz oder Blutung wurde nach präoperativer Chemotherapie im Vergleich zu den nur operierten Patienten nicht erhöht, die Insuffizienzrate lag bei $3 / 26$ und 4/38 Patienten. Die lokale Tumorkontrolle war nach Chemotherapie günstiger. Ein lokales Tumorrezidiv trat nach Chemotherapie weniger häufig auf $(n=3 / 26)$ als nach alleiniger Operation (10/38) und Rezidive in Form von Lymphknotenmetastasen traten nur bei den alleinig Operierten auf.

Die Prognose des Gesamtkollektivs wurde durch die Chemotherapie nicht beeinflusst; die 5-JÜR von 37,5\% nach Chemotherapie unterschied sich nicht signifikant von den 42,3\% nur nach Operation. Auch für Responder konnte in der Subgruppenanalyse mit einer 5-JÜR von $44,6 \%$ kein signifikanter Überlebensvorteil gegenüber den nur Operierten mit 42,3\% und den Nicht-Respondern mit 20\% erreicht werden. Durch die neoadjuvante Chemotherapie nach PELF wird die lokale Tumorkontrolle, nicht jedoch die Prognose beim Magenkarzinom verbessert. 


\section{Literaturverzeichnis}

1. Ajani JA, Mansfield PF, Janjan N, Morris J, Pisters PW, Lynch PM, Feig B, Myerson R, Nivers R, Cohen DS, Gunderson LL (2004) Multi-institutional trial of preoperative chemoradiotherapy in patients with potentially resectable gastric carcinoma. J Clin Oncol.; 22(14):2774-80

2. AWMF online-Leitlinie Chirurgie/Onkologie: Magenkarzinom

3. Bentrem D, Wilton A, Mazumdar M, Brennan M, Coit D (2004) The value of peritoneal cytology as a preoperative predictor in patients with gastric carcinoma undergoing a curative resection. Ann Surg Oncol.; 12(5):347-53

4. Biffi R, Fazio N, Luca F, Chiappa A, Andreoni B, Zampino MG, Roth A, Schuller JC, Fiori G, Orsi F, Bonomo G, Crosta C, Huber O. (2010) Surgical outcome after docetaxel-based neoadjuvant chemotherapy in locallyadvanced gastric cancer. World J Gastroenterol.; 21;16(7):868-74

5. Brennan MF (1995) Benefit of aggressive multimodality treatment for gastric cancer. Ann Surg Oncol.; 2(4):286-7

6. Burke EC, Karpeh MS Jr, Conlon KC, Brennan MF (1998) Peritoneal lavage cytology in gastric cancer: an independent predictor of outcome. Ann Surg Oncol.; 5(5):411-5

7. Cascinu S, Graziano F, Barni S, Labianca R, Comella G, Casaretti R, Frontini L, Catalano V, Baldelli AM, Catalano G. (2001) A phase II study of sequential chemotherapy with docetaxel after the weekly PELF regimen in advanced gastric cancer. A report from the Italian group for the study of digestive tract cancer. Br J Cancer.; 84(4):470-4

8. Cheong JH, Shen JY, Song CS, Hyung WJ, Shen JG, Choi SH, Noh SH (2006) Early postoperative intraperitoneal chemotherapy following cytoreductive surgery in patients with very advanced gastric cancer. Ann Surg Oncol.; 14(1):61-8

9. Chipponi J, Huguier M, Pezet D, Basso N, Hay JM, Quandalle P, Jaeck D, Fagniez PL, Gainant A (2004) Randomized trial of adjuvant chemotherapy after curative resection for gastric cancer. Am J Surg.; 187(3):440-5

10. Crabtree JE, Court M, Aboshkiwa MA, Jeremy AH, Dixon MF, Robinson PA (2004) Gastric mucosal cytokine And epithelial response to Helicobacter pylori infection in Mongolian gerbils. J Pathol.; 202(2):197-207 
11. Cunningham D, Allum WH, Stenning SP, Thompson JN, Van de Velde CJ, Nicolson M, Scarffe JH, Lofts FJ, Falk SJ, Iveson TJ, Smith DB, Langley RE, Verma M, Weeden S, Chua YJ, MAGIC Trial Participants (2006) Perioperative chemotherapy versus surgery alone for resectable gastroesophageal cancer. N Engl J Med.; 355(1):11-20

12. Douglass HOJr. (2003) Detection and treatment of the micrometastases of gastric cancer. Ann Surg Oncol.; 11(1):2-3

13. Drews $\mathrm{R}$, Goral $\mathrm{R}$ Über die lymphogene Metastasierung des Magenkarzinoms. Langenbecks Arch.klin.Chir.; Bd.295:125-129

14. Drognitz O , Henne K, Weissenberger C, Bruggmoser G, Göbel H, Hopt UT, Frommhold H, Ruf G (2007) Long-Term Results After Intraoperative Radiation Therapy For Gastric Cancer. Int. J. Radiation Onc. Biol. Phys: 1;70(3):715-21

15. Edwards P, Blackshaw GR, Lewis WG, Barry JD, Allison MC, Jones DR. (2004) Prospective comparison of D1 vs modified D2 gastrectomy for carcinoma. Br. J. Cancer; 90(10):1888-92

16. Elaraj DM, Ettinghausen SE (2005) Cytological analysis of peritoneal washings: now part of the standard preoperative staging evaluation for patients with resectable gastric cancer. Ann Surg Oncol.; 12(5):339-41

17. Farthmann EH, Kirchner R (1992) Stomach carcinoma: surgical strategy and therapy results. Schweiz Rundsch Med Prax.; 81(31-32):938-40

18. Farthmann EH, Ruf G Magenkarzinom: Gastrektomie, Lymphadenektomie, IORT

19. Fink U, Stein HJ, Siewert JR (1998) Multimodal therapy of tumors of the upper gastrointestinal tract. Chirurg; 69(4):349-59

20. Fujimoto S, Akao T, Itoh B, Koshizuka I, Koyano K (1977) Protracted oral chemotherapy with fluorinated pyrimidines as an adjuvant to surgical treatment for stomach cancer. Ann Surg.; 185(4):462-6

21. Gallardo-Rincón D, Oñate-Ocaña LF, Calderillo-Ruiz G 1999) Neoadjuvant chemotherapy with P-ELF (cisplatin, etoposide, leucovorin, 5-fluorouracil) followed by radical resection in patients with initially unresectable gastric adenocarcinoma: a phase II study. Ann Surg Oncol; 7(1):45-50

22. Glas J, Török HP, Schneider A, Brünnler G, Kopp R, Albert ED, Stolte M, Folwaczny $C$ (2004) Allele 2 of the interleukin-1 receptor antagonist gene is associated with early gastric cancer. $\mathrm{J}$ Clin Oncol.; 22(23):4746-52 
23. Gold JS, Jaques DP, Bentrem DJ, Shah MA, Tang LH, Brennan MF, Coit DG (2006) Outcome of patients with known metastatic gastric cancer undergoing resection with therapeutic intent. Ann Surg Oncol.; 14(2):365-72

24. Gumpp V, Henss H (2007) Comprehensive Cancer Center Freiburg Magenkrebs Klinisches Krebsregister

25. Hallissey MT, Dunn JA, Ward LC, Allum WH (1994) The second British Stomach Cancer Group trial of adjuvant radiotherapy or chemotherapy in resectable gastric cancer: five-year follow-up. Lancet; 343(8909):1309-12

26. Kim S, Lim DH, Lee J, Kang WK, MacDonald JS, Park CH, Park SH, Lee SH, $\underline{\text { Kim K}}, \underline{\text { Park JO }}$, Kim WS, Jung CW, Park YS, Im YH, Sohn TS, Noh JH, $\underline{\text { Heo }}$ JS, Kim YI, Park CK, Park K. (2005) An observational study suggesting clinical benefit for adjuvant postoperative chemoradiation in a population of over 500 cases after gastric resection with D2 nodal dissection for adenocarcinoma of the stomach. Int J Radiat Oncol Biol Phys.; 1;63(5):1279-85

27. Klinikarzt (2008); 37 (7+8): 366-371

28. Lake JC, Lopez PP (2004) Gastric Cancer: Diagnosis and Treatment Options. American Family Physician; Vol. 69, Number 5: 1133-1140

29. Lee KA, Ki CS, Kim HJ, Sohn KM, Kim JW, Kang WK, Rhee JC, Song SY, Sohn TS (2004) Novel interleukin 1beta polymorphism increased the risk of gastric cancer in a Korean population. J Gastroenterol.; 39(5):429-433

30. Lee KJ, Inoue M, Otani T, Iwasaki M, Sasazuki S, Tsugane S; JPHC Study Group (2006) Gastric cancer screening and subsequent risk of gastric cancer: a large-scale population-based cohort study, with a 13-year follow-up in Japan. Int J Cancer; 118(9):2315-21

31. Lim L, Michael M, Mann GB, Leong T (2005) Adjuvant therapy in gastric cancer. J of Clin Oncol.; 23(25):6220-32

32. Lowy AM, Feig BW, Janjan N, Rich TA, Pisters PW, Ajani JA, Mansfield PF (2001) A pilot study of preoperative chemoradiotherapy for resectable gastric cancer. Ann Surg Oncol.; 8(6):519-24

33. Macdonald JS, Fleming TR, Peterson RF, Berenberg JL, McClure S, Chapman RA, Eyre HJ, Solanki D, Cruz AB Jr, Gagliano R, et al. (1995) Adjuvant chemotherapy with 5-FU, adriamycin, and mitomycin-C (FAM) versus surgery alone for patients with locally advanced gastric adenocarcinoma: A Southwest Oncology Group study. Ann Surg Oncol.; 2(6):488-94 
34. Macdonald JS, Smalley SR, Benedetti J, Hundahl SA, Estes NC, Stemmermann GN, Haller DG, Ajani JA, Gunderson LL, Jessup JM, Martenson JA. (2001) Chemoradiotherapy after surgery compared with surgery alone for adenocarcinoma of the stomach or gastroesophageal junction. N Engl J Med.; 6;345(10):725-30

35. Maley CC, Glaipeau PC, Li X, Sanchez CA, Paulson TG, Blount PL, Reid BJ (2004) The Combination of genetic instability and clonal expansion predicts progression to eosophageal adenocarinoma. Cancer Res; 64(20):7629-2633

36. Matsuhisa T, Matsukura N, Yamada N (2004) Topography of chronic active gastritis in Helicobacter pylori-positive Asian populations: age-, gender-, and endoscopic diagnosis-matched study. J Gastroenterol.; 39(4):324-8

37. Mori T, Fujiwara $\mathrm{Y}$, Sugita $\mathrm{Y}$, Azama $\mathrm{T}$, Ishii $\mathrm{T}$, Taniguchi K, Yamazaki K, Takiguchi S, Yasuda T, Yano M, Monden M (2003) Application of molecular diagnosis for detection of peritoneal micrometastasis and evaluation of preoperative chemotherapy in advanced gastric carcinoma. Ann Surg Oncol.;11(1):14-20

38. Nakajima T, Kinoshita T, Nashimoto A, Sairenji M, Yamaguchi T, Sakamoto J, Fujiya T, Inada T, Sasako M, Ohashi Y; National Surgical Adjuvant Study of Gastric Cancer Group (2007) Randomized controlled trial of adjuvant uraciltegafur versus surgery alone for serosa-negative, locally advanced gastric cancer. British J of Surgery; 94(12):1468-76

39. Nakajima T, Ota K, Ishihara S, Oyama S, Nishi M, Ohashi Y, Yanagisawa A (1997)Combined intensive chemotherapy and radical surgery for incurable gastric cancer. Ann Surg Oncol.; 4(3):203-8

40. Nardone G, Rocco A, Vaira D, Staibano S, Budillon A, Tatangelo F, Sciulli MG, Perna F, Salvatore G, Di Benedetto M, De Rosa G, Patrignani P (2004). Expression of COX-2, mPGE-synthase1, MDR-1 (P-gp), and Bcl-xL: a molecular pathway of $\mathrm{H}$ pylori-related gastric carcinogenesis. J Pathol.; 202(3):305-312

41. Nitti D, Wils J, Dos Santos JG, Fountzilas G, Conte PF, Sava C, Tres A, Coombes RC, Crivellari D, Marchet A, Sanchez E, Bliss JM, Homewood J, Couvreur ML, Hall E, Baron B, Woods E, Emson M, Van Cutsem E, Lise M; EORTC GI Group; ICCG (2006) Randomized phase III trials of adjuvant FAMTX or FEMTX compared with surgery alone in resected gastric cancer. A 
combined analysis of the EORTC GI Group and the ICCG. Ann Oncol.; 17(2):262-9

42. Okuda M, Miyashiro E, Nakazawa T (2004) Helicobacter pylori infection in childhood. J.Gastroenterol.; 39(8):809-810

43. Oñate-Ocaña LF, Aiello-Crocifoglio V, Mondragón-Sánchez R, Ruiz-Molina M (1999)Survival benefit of D2 lympadenectomy in patients with gastric adenocarcinoma. Ann Surg Oncol.; 7(3):210-7

44. Orditura M, De Vita F, Muto P, Vitiello F, Murino P, Lieto E, Vecchione L, Romano A, Martinelli E, Renda A, Ferraraccio F, Del Genio A, Ciardiello F, Galizia G. (2010) Adjuvant chemoradiotherapy in patients with stage III or IV radically resected gastric cancer: a pilot study. Arch Surg. Mar.; 145(3):239

45. Paramo JC, Gomez G (1999) Dynamic CT in the preoperative evaluation of patients with gastric cancer: correlation with surgical findings and pathology. Ann Surg Oncol.; 6(4):379-84

46. Philip Agop Philip Improvements in Gastric Cancer Therapy Options American Society og Clinical Oncology $39^{\text {th }}$ Annual Meeting

47. Preiß J, Dornoff W, Hagmann FG, Schmieder A (2008) Onkologie Interdisziplinäre Empfehlungen zur Therapie

48. Rohde H, Gebbensleben B, Bauer P, Stützer H, Zieschang J. (1987) Has there been any improvement in the staging of gastric cancer? Findings from the German Gastric Cancer TNM Study Group. Cancer; 64(12):2465-81

49. Roukos DH, Kappas AM (2001) Limitations in controlling risk for recurrence after curative surgery for advanced gastric cancer are Now well-explained by molecular-based mechanism. Ann Surg Oncol.; 8(8):620-1

50. Sakuramoto S, Sasako M, Yamaguchi T, Kinoshita T, Fujii M, Nashimoto A, Furukawa $\mathrm{H}$, Nakajima $T$, Ohashi $\mathrm{Y}$, Imamura $\mathrm{H}$, Higashino $\mathrm{M}$, Yamamura $\mathrm{Y}$, Kurita A, Arai K; ACTS-GC Group (2007) Adjuvant chemotherapy for gastric cancer with S-1, an oral fluoropyrimidine. N Engl J Med; 357(18):1810-20

51. Samson PS, Escovidal LA, Yrastorza SG, Veneracion RG, Nerves MY (2002) Re-study of gastric cancer: analysis of outcome. World J Surg.; 26(4):428-33

52. Sastre J, Garcia-Saenz JA, Diaz-Rubio E (2006) Chemotherapy for gastric cancer. world J Gastroenterol:; 12(2):204-13

53. Schwarz RE, Smith DD (2007) Clinical impact of lymphadenectomy extent in resectable gastric cancer of advanced stage. Ann Surg Oncol.; 14(2):317-28 
54. Sendler A, Prinz C, Janetschek P, Becker K, Grützner U, Lordick F, Schuhmacher C, Zimmermann F. (2006) Magenkarzinom. Manual Gastrointestinale Tumoren; 7:26-51

55. Solomon NL, Cheung MC, Byrne MM, Zhuge Y, Franceschi D, Livingstone AS, Koniaris LG. (2010) Does chemoradiotherapy improve outcomes for surgically resected adenocarcinoma of the stomach or esophagus? Ann Surg Oncol.; 17(1):98-108

56. Songum I, Van de Velde CJH (1996) Can Surgical Treatment Results in Gastric Cancer Be Improved? The Oncoligist: Vol.1, No 1_2:36-40

57. Sue-Ling HM (1998) Detection and treatment of early gastric cancer in the West. Gastric cancer; 1(1): 8-9

58. Tashiro A, Sano M, Kinameri K, Fujita K, Takeuchi Y (2006) Comparing mass screening techniques for gastric cancer in Japan. World J Gastroenterol.; 12(30):4873-4

59. Tatsuguchi A, Miyake K, Gudis K, Futagami S, Tsukui T, Wada K, Kishida T, Fukuda Y, Sugisaki Y, Sakamoto C (2004). Effect of Helicobacter pylori infection on ghrelin expression in human gastric mucosa. Am J Gastroenterol.: 99(11):2121-7

60. Wanke M, Schwan H (1979) Pathology of gastric cancer. World J Surg.; 3(6):675-84

61. Yao JC, Ajani JA (2002) Therapy of localized gastric cancer: preoperative and postoperative approaches. Ann Oncol.; 13(4):7-12

62. Yoshikawa T, Sasako M, Yamamoto S, Sano T, Imamura H, Fujitani K, Oshita $\underline{H}$, Ito $S$, Kawashima $\mathrm{Y}$, Fukushima N (2009) Phase II study of neoadjuvant chemotherapy and extended surgery for locally advanced gastric cancer. $\mathrm{Br}$ Surg.; 96(9):1015-22

63. Yoshikawa T, Tsuburaya A, Morita S, Kodera $\mathrm{Y}$, Ito $\mathrm{S}$, Cho H, Miyashita $\mathrm{Y}$, Sakamoto J (2010) A Comparison of Multimodality Treatment: Two or Four Courses of Paclitaxel plus Cisplatin or S-1 plus Cisplatin Followed by Surgery for Locally Advanced Gastric Cancer, a Randomized Phase II Trial (COMPASS). Jpn J Clin Oncol 


\section{Anhang}

\section{Brief/Fragebogen an Hausärzte}

Sehr geehrter Herr Kollege,

über die Behandlungsergebnisse nach Gastrektomie bei Magenkarzinom mit und ohne Vorbehandlung gibt es in der Literatur unterschiedliche Ergebnisse hinsichtlich der Rezidivrate bzw. des Überlebens der Patienten. Wir möchten um ihre Unterstützung bitten.

Wir haben den Patienten ... im Jahre... operiert. Die adjuvante Therapie wurde nicht in unserer Klinik durchgeführt. Wir möchten Sie bitten, uns Informationen zur nichtoperativen onkologischen Behandlung des Patienten zukommen zu lassen. Und zwar:

- Behandlungszeitraum und Dauer

- Wo behandelt? (Krankenhaus etc.)

- Art der Therapie: Chemotherapie/Radiatio/kombiniert

- Welches Schema:

- Radiatio: Gesamtdosis/Vollständigkeit/Unterbrechung der Therapie

- Chemotherapie: Substanzen/Vollständigkeit?

- Nebenwirkungen

Über einen entsprechenden Befund- bzw. Behandlungsbericht wären wir Ihnen sehr dankbar. Gerne können Sie uns auch diesen Brief stichwortartig ergänzen bzw. Zutreffendes anstreichen und an uns (gerne auch per Fax) zurückschicken. Nach Abschluss der Untersuchung werden wir Ihnen über die Ergebnisse berichten.

Wir danken für Ihnen bereits jetzt für Ihre freundliche Unterstützung. 


\section{Danksagung}

Mein besonderer Dank gilt Professor Dr. Thomusch der meine Doktorarbeit als Erstgutachter betreut hat und mit seinem Wissen und Rat zu einem guten Abschluss der Arbeit beigetragen hat.

Ebenfalls möchte ich mit bei PD Dr. Illerhaus Bedanken, der die Aufgabe des Zweitgutachters freundlicherweise übernommen hat.

Ein ganz herzliches Dankeschön möchte ich an Dr. Goos richten, der mir von Beginn meiner Doktorarbeit an hilfreiche Unterstützung gegeben hat.

Danke sagen möchte ich Frau Eva Fischer, die mir in den statistischen Fragen in Rat und Tat zur Seite gestanden ist und Dr. Makowiec, der mich außerordentlich bei der Statistik unterstützt hat.

Danken möchte ich meiner Familie, die mich von den Anfängen bis zur endgültigen Fertigstellung der Doktorarbeit, unterstützt hat. 